\title{
Splines and index theorem
}

\section{Procesi}

Received: 16 May 2011 / Revised: 18 July 2011 / Accepted: 17 August 2011 /

Published online: 30 August 2011

(C) The Author(s) 2011. This article is published with open access at SpringerLink.com

\begin{abstract}
In this article we want to present a meeting ground between rather different mathematical topics from numerical analysis to index theory and symplectic geometry. The unifying idea is that of linear representation of tori inside which the combinatorics, analysis and geometry is developed.
\end{abstract}

\section{Contents}

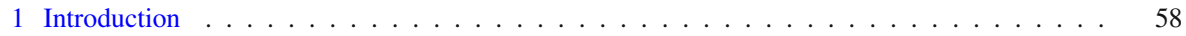

Part 1. Polytopes and splines . . . . . . . . . . . . . . . . . . . . . . . . 64

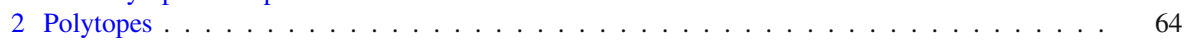

2.1 From a list of vectors . . . . . . . . . . . . . . . . . . . . . 64

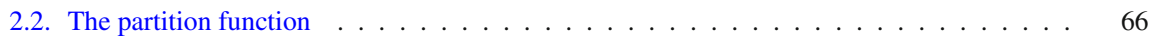

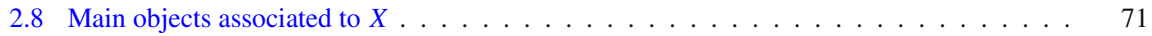

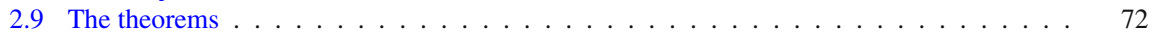

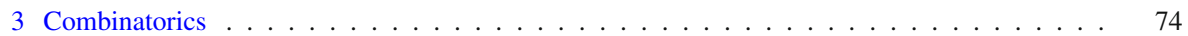

3.1 Unbroken bases . . . . . . . . . . . . . . . . . . . . . . . . . . 74

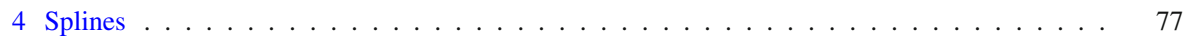

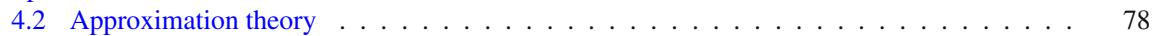

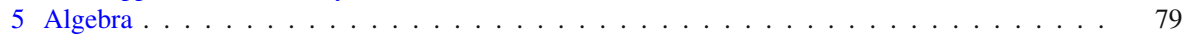

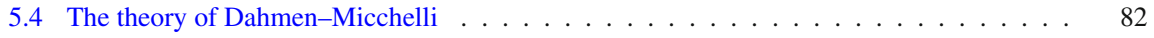

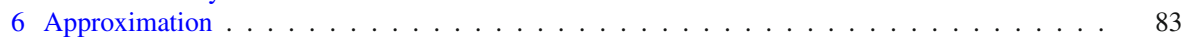

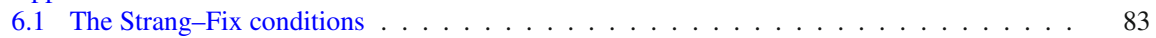

Part 2. Arithmetic and combinatorics . . . . . . . . . . . . . . . . . . . . . . 86

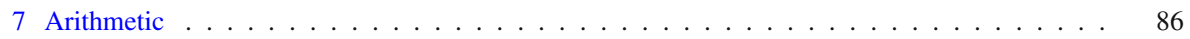

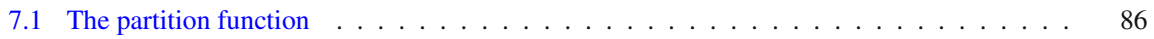

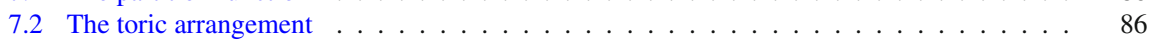

Communicated by E. Zelmanov.

C. Procesi $(\varangle)$

Università di Roma, La Sapienza, Rome, Italy

e-mail: procesi@mat.uniroma1.it 


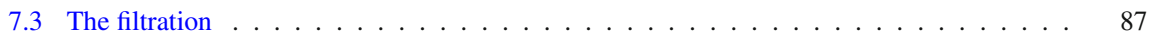

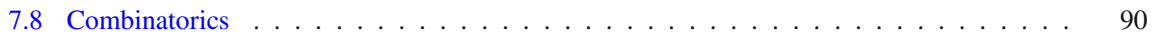

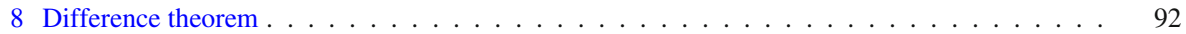

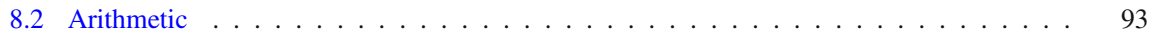

9 Residues ................................... 94

9.1 Wonderful models . . . . . . . . . . . . . . . . . . . . . . . . . . . 94

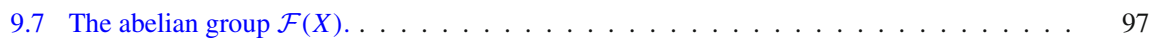

9.13 Localization theorem . . . . . . . . . . . . . . . . . . . . . . . . . . . . . . . . . 99

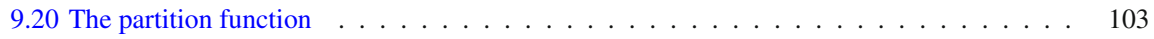

Part 3. Index theory . . . . . . . . . . . . . . . . . . . . . . . . . 103

10 The Atiyah-Singer index theorem . . . . . . . . . . . . . . . . . . 103

10.1 Equivariant $K$-theory . . . . . . . . . . . . . . . . . . . . . . . . . . 103

10.3 Transversally elliptic operators . . . . . . . . . . . . . . . . . . . . . . . . 105

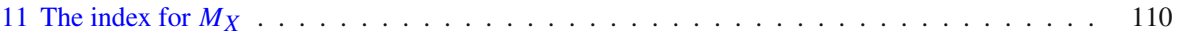

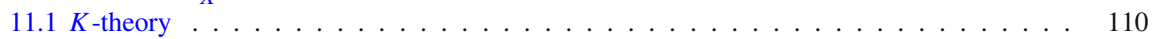

12 Generators . . . . . . . . . . . . . . . . . . . . . 116

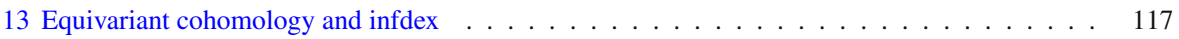

13.1 Equivariant de Rham cohomology . . . . . . . . . . . . . . . . . . . . . . . . . 117

13.3 Action form and the moment map . . . . . . . . . . . . . . . . . . 118

13.8 Infinitesimal index . . . . . . . . . . . . . . . . . . . . . . . . . 119

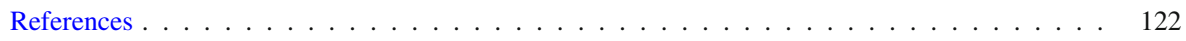

\section{Introduction}

In 1968 appears the fundamental work of Atiyah and Singer on the index theorem of elliptic operators, a theorem formulated in successive steps of generality [3-7].

This theorem is the crowning point of a long sequence of ideas starting from the theory of algebraic functions of Riemann and passing through the theory of De Rham and Hodge on cohomology on manifolds. The index theorem generalizes the RiemannRoch theorem of Hirzebruch, a cornerstone in modern algebraic geometry [25].

What is an index? Recall that the index $i(A)$ of a linear operator, when defined, is $i(A):=\operatorname{dim}(\operatorname{ker} A)-\operatorname{dim}\left(\operatorname{ker} A^{*}\right)$, where $\operatorname{ker}(A)$ is the space of solutions of the homogeneous equation $A u=0$ and $A^{*}$ denotes the adjoint.

- The linear operators consider by Atiyah and Singer are differential (or rather pseudodifferential) operators on manifolds.

- Precisely they operate not simply on functions but, as usual in global geometry, on sections of vector bundles.

The condition of being elliptic (like the Laplace operator) is needed to insure that the index is well defined and a finite integer. The index theorem produces a formula for the index through cohomological data associated to the operator, the manifold and the bundles involved (Chern character and Todd class).

\section{The need for $K$-theory}

An essential step in the index theory is given by the construction of a generalized cohomology theory, called $K$-theory.

Then the index theorem passes through several steps:

- to an operator one associates the symbol $\Sigma$, which is a (matrix valued) function on the cotangent bundle.

- to the symbol an element of a suitable group of $K$-theory, the index can be computed this way. 
- Alternatively from $K$-theory one passes to cohomology using the Chern character $\operatorname{ch}(\Sigma)$ of $\Sigma$.

- The final formula is proved by showing enough properties of all these steps which reduce the formula to some basic cases.

One general and useful setting is for operators on a manifold $M$ which:

(i) satisfy a symmetry with respect to a compact Lie group $G$

(ii) are elliptic in directions transverse to the $G$-orbits.

In this case the values of the index are generalized functions on $G$. In fact in this case the kernel of $A$ or of $A^{*}$ is no more finite dimensional, but it is a particularly well behaved representation $\rho: G \rightarrow U(\mathbb{H})$, called of trace class, from the group of symmetries $G$ to unitary operators on a Hilbert space. This means that we can integrate the $C^{\infty}$ functions of $G$ to operators $T_{f}:=\int_{G} f(g) \rho(g)$ which have a trace and $f \mapsto \operatorname{Tr}\left(T_{f}\right)$ is a distribution.

Being of trace class implies that both spaces of solutions are

- a (possibly infinite) direct sum of irreducible representations of $G$ each with finite multiplicity

- the multiplicities have some moderate growth so that they can be interpreted as Fourier coefficients of generalized functions (distributions) on $G$.

The analytic index of the operator $A$ is the virtual trace class representation of $G$, obtained as difference of the spaces of solutions of $A$ and its adjoint $A^{*}$ in an appropriate Sobolev space. This is equivalently described by an integral valued function on the set of irreducible representations, the index multiplicity.

The main topological ingredient of the theory is the group $K_{G, c}^{0}\left(T_{G}^{*} M\right)$ of equivariant $K$-theory with compact support, which is defined in a topological fashion.

If $T^{*} M$ is the cotangent bundle. we denote by $T_{G}^{*} M$ the closed subset of $T^{*} M$, union of the conormals to the $G$ orbits. Using a Riemannian metric we may replace the cotangent bundle with the tangent bundle, and then conormal is replaced with orthogonal.

Example $1.1 G=S^{1}$ acting by rotations on the plane $\mathbb{R}^{2}$, the orbits are the circles centered at the origin, we identify $T^{*} M=\mathbb{R}^{4}$ with pairs of vectors.

A pair of vectors $(a, b) \in \mathbb{R}^{2} \times \mathbb{R}^{2}$ is in $T_{S^{1}}^{*} M$ if either $a=0$, in this case its orbit reduces to 0 and $b$ arbitrary, or $a \neq 0$ its orbit is a circle and $b$ is orthogonal to the orbit, that is it is proportional to $a$.

Assume first that $M$ is compact. The condition of being transversally elliptic is expressed by the fact that the symbol $\Sigma$ is invertible on $T_{G}^{*} M$ minus the zero section. So one repeats the analysis for the index, substituting to $K$-theory equivariant $K$-theory.

This index depends only of the class defined by $\Sigma$ in $K_{G, c}^{0}\left(T_{G}^{*} M\right)$, so that in the end the index defines a $R(G)$ module homomorphism from $K_{G, c}^{0}\left(T_{G}^{*} M\right)$ to virtual trace class representations of $G$.

Alternatively, from equivariant $K$-theory one passes to equivariant cohomology with compact supports using the equivariant Chern character $\operatorname{ch}(\Sigma)$ of $\Sigma$. This is an element of a suitable $G$-equivariant cohomology group, with compact supports, of $T_{G}^{*} M$. 
In his Lecture Note describing joint work with I.M. Singer, Elliptic operators and compact groups, [1], Atiyah explains how to reduce general computations to the case in which

- $G$ is a torus, e.g. $G=\left(S^{1}\right)^{s}, \hat{G}=\mathbb{Z}^{s}$ its character group.

- The manifold $M$ is a complex linear representation $M_{X}=\oplus_{a \in X} L_{a}$,

- $X \subset \hat{G}=\mathbb{Z}^{s}$ is a finite list of characters and $L_{a}$ denotes the one dimensional complex line where $G$ acts by the character $a \in X$.

He then computes explicitly in several cases and ends his introduction saying “...for a circle (with any action) the results are also quite explicit. However for the general case we give only a reduction process and one might hope for something explicit. This probably requires the development of an appropriate algebraic machinery, involving cohomology but going beyond it."

A solution to this question is given in the paper Vector partition functions and index of transversally elliptic operators, by C. De Concini, C. Procesi, M. Vergne [20].

In this paper I want to give an idea of the algebraic machinery which provides a complete solution to this question. This turns out to be a spinoff of the theory of splines, a developement of the classic Theory of Schoenberg, [31] as in the classic book [15] by de Boor, Höllig, and Riemenschneider. For most of this material the reader can consult the recent book by De Concini, and Procesi Topics in hyperplane arrangements, polytopes and box-splines [23].

The main goal is to describe explicitly the map induced by the index, from $K_{G, c}^{0}\left(T_{G}^{*} M\right)$ to virtual trace class representations of $G$. This is described combinatorially.

Once we identify the character group of $G$ with the free abelian group $\mathbb{Z}^{s}$ we have, as values of the index, a space of integer valued functions on $\mathbb{Z}^{s}$. Our main result is for a linear representation $M_{X}$ associated to a list of characters $X$ in $\mathbb{Z}^{s}$ :

- We identify the space of functions, image of the index, for $M=M_{X}$.

- We prove that the index is an isomorphism between $K_{G, c}^{0}\left(T_{G}^{*} M\right)$ and its image in the space of functions.

We need the basic notion of Partition function for a list $X$ of vectors in $\mathbb{Z}^{s}$.

If there is a linear form $\phi$ so that $\langle\phi \mid a\rangle>0, \forall a \in X$ we have a $\mathbb{Z}$ valued function $P_{X}(b)$ on $\mathbb{Z}^{s}$ defined as:

$$
P_{X}(b):=\#\left\{\left(t_{1}, \ldots, t_{m}\right) \in \mathbb{N}^{m} \mid \sum_{i=1}^{m} t_{i} a_{i}=b\right\}
$$

The function $P_{X}(b)$ is best expressed via its generating series

$$
\sum_{b \in \mathbb{Z}^{s}} P_{X}(b) e^{b}=\prod_{a \in X} \frac{1}{1-e^{a}} .
$$

If $X$ is the list of characters of a linear representation $M_{X}$ the partition function can be viewed as the trace of the representation of $S^{S}$ in the symmetric algebra $S\left(M_{X}\right)$. 
Atiyah-Singer constructed a "pushed" $\bar{\partial}$ operator on $M_{X}$, with index the partition function.

For a general list $X$ consider a linear function $\phi$ which is non-zero on each element of $X$, then $X$ is divided into two parts $A$ where $\phi$ is positive, and $B$ respectively where $\phi$ is negative. We may thus consider the partition function $P_{A,-B}$.

Lemma 1.2 The function $P_{A,-B}$ depends only on the chamber $F$, of the hyperplane arrangement defined by $X$ in the dual space, in which $\phi$ lies and will be denoted $P_{X}^{F}$.

Each of these partition functions can be viewed as multiplicity index of a "pushed" $\bar{\partial}$ operator on $M_{X}$ by changing the complex structure.

Integer valued functions on $\mathbb{Z}^{s}$ are a module (using translation) over the group algebra $\mathbb{Z}\left[\mathbb{Z}^{s}\right]$ (also denoted by $R(T)$, if $T$ is the torus with character group $\hat{T}=\mathbb{Z}^{s}$ ). We can thus consider the $\mathbb{Z}\left[\mathbb{Z}^{s}\right]$ module $\tilde{\mathcal{F}}(X)$ of functions on $\mathbb{Z}^{s}$ generated by all the partition functions $P_{A,-B}$ for all the chambers.

The main Theorem 1 states that the index induces an isomorphism between $K_{G, c}^{0}\left(T_{G}^{*} M_{X}\right)$ and $\tilde{\mathcal{F}}(X)$. Moreover there is a very precise description of the group $\tilde{\mathcal{F}}(X)$.

1.2.1. The case $S^{1}$. For $s=1$, or $G=S^{1}$ we denote by $t$ the basic character of $S^{1}:=\{t|| t \mid=1\}$, so that $R\left(S^{1}\right)=\mathbb{Z}\left[t, t^{-1}\right]$. We write generalized functions by their generating series $\sum_{n \in \mathbb{Z}} a_{n} t^{n}$.

When $S^{1}$ acts by homotheties on $\mathbb{C}^{k+1}$ we have $\mathbb{C}^{k+1}=M_{X}$ and $X=$ $[t, t, \ldots, t], k+1$ times. We start by describing Theorem 1 in this special case.

By the "pushed" $\bar{\partial}$ operator we get the generalized function

$$
\Theta_{X}(t):=\sum_{n=0}^{\infty}\left(\begin{array}{c}
n+k \\
k
\end{array}\right) t^{n}
$$

where

$$
\left(\begin{array}{c}
n+k \\
k
\end{array}\right)=\frac{(n+k)(n+k-1) \ldots(n+1)}{k !}, n \geq 0
$$

is the partition function for $X=\underbrace{\{1, \ldots, 1\}}_{k+1}$.

In this case we only have two chambers and the other partition function

$$
\left(\begin{array}{c}
-n+k \\
k
\end{array}\right), n \leq 0
$$

for $X=\underbrace{\{-1, \ldots,-1\}}_{k+1}$.

The map $n \mapsto\left(\begin{array}{c}n+k \\ k\end{array}\right)$ is a polynomial function on $\mathbb{Z}$ which, for any $n$ positive or negative, represents the dimension of a virtual space, the alternate sum of the cohomology spaces of the sheaf $\mathcal{O}(n)$ on $k$-dimensional projective space. 
One easily sees that this function gives the Fourier coefficients of a generalized function $\theta_{k}(t):=\sum_{n=-\infty}^{\infty}\left(\begin{array}{c}n+k \\ k\end{array}\right) t^{n}$, on $S^{1}$ supported at $t=1$. In fact for $k=0$ the generalized function $\theta_{0}(t):=\sum_{n=-\infty}^{\infty} t^{n}$, is the Fourier expansion of the delta function $\delta_{1}$ and we obtain $\theta_{k}(t)$ by applying suitable derivatives to $\theta_{0}(t)$.

One can prove that the tangential Cauchy-Riemann operator, on the unit sphere $S_{2 k+1}$ of $\mathbb{C}^{k+1}$, is a transversally elliptic operator with index $\theta_{k}(t)$.

Proposition 1.3 The index map is an isomorphism from $K_{S^{1}, c}^{0}\left(T_{S^{1}}^{*} M_{X}\right)$ to the space $\tilde{\mathcal{F}}(X)$ of generalized functions on $S^{1}$ generated by $\Theta_{X}$ and $\theta_{X}=\theta_{k}(t)$ under multiplication by elements of $R\left(S^{1}\right)=\mathbb{Z}\left[t, t^{-1}\right]$.

In fact the $R\left(S^{1}\right)$ module generated by $\Theta_{X}$ is free over $R\left(S^{1}\right)$.

The $R\left(S^{1}\right)$ module generated by $\theta_{X}$ is the torsion submodule.

- This submodule is the module of polynomial functions on $\mathbb{Z}$ of degree at most $k$ so it is a free $\mathbb{Z}$-module of rank $k+1$.

- It corresponds to indices of operators on $\mathbb{C}^{k+1}-\{0\}$, the set where $S^{1}$ acts freely

- It is the space of solutions of the difference equation $\nabla^{k} f=0$, where we define the operator $\nabla$ by $(\nabla f)(n)=f(n)-f(n-1)$.

1.3.1. Higher dimension. In higher dimensions, the single difference equation $\nabla^{k} f=0$ must be replaced by a system of difference equations, discovered by Dahmen-Micchelli as the natural generalization of a system of differential equations associated to splines in approximation theory.

We need a basic definition of combinatorial nature. Consider a sequence $X$ of integral vectors (i.e. weights of a torus $G$ with character group $\Lambda$ ).

Definition 1.4 We say that a sublist $Y \subset X$ is a cocircuit, if the elements in $X \backslash Y$ do not span $V$ and $Y$ is minimal with this property.

For an integral vector $a \in \Lambda$ (often identified with $\mathbb{Z}^{s}$ ) define the translation operator $\tau_{a}$ and the difference operator $\nabla_{a}$ by

$$
\tau_{a}(f)(x):=f(x-a), \quad \nabla_{a}=1-\tau_{a} .
$$

These operators act on functions on $\mathbb{Z}^{s}$. For a list $Y$ of integral vectors we set $\nabla_{Y}:=$ $\prod_{a \in Y} \nabla_{a}$ and introduce thus the system of difference equations $\nabla_{Y} f=0$ associated to cocircuits $Y$ of $X \subset \mathbb{Z}^{s}$. The space of solutions is denoted by $D M(X)$. There is a beautiful Theory of this space which we will in part recall presently (cf. Definition 7.5).

Recall the $\mathbb{Z}\left[\mathbb{Z}^{s}\right]$ module $\tilde{\mathcal{F}}(X)$ of functions on $\mathbb{Z}^{s}$ generated by all the partition functions $P_{A,-B}$ for all the chambers.

Theorem 1 The index induces an isomorphism between $K_{G, c}^{0}\left(T_{G}^{*} M_{X}\right)$ and $\tilde{\mathcal{F}}(X)$.

Moreover there is a very precise description of the group $\tilde{\mathcal{F}}(X)$.

The main result is about the open set $M_{X}^{f}$ of $M_{X}$ where $G$ acts with finite stabilizers. 
Theorem 2 The index map induces an isomorphism between $K_{G, c}^{0}\left(T_{G}^{*} M_{X}^{f}\right)$ and the space $D M(X)$.

For the general case we need some associated spaces of functions, generalizing $D M(X)$. We thus introduce the spaces $\tilde{\mathcal{F}}_{i}(X)$ associated to the orbit types in $M_{X}$.

Any linear subspace $\underline{r}$ generated by vectors in $X$ is called a rational subspace, we denote by $S_{X}$ the set of all rational subspaces. We define:

$$
\mathcal{F}(X):=\left\{f \mid \nabla_{X \backslash \underline{r}} f \text { is supported on } \underline{r} \text { for every rational subspace } \underline{r}\right\} .
$$

Clearly $D M(X)$ is contained in $\mathcal{F}(X)$.

One can show that $\tilde{\mathcal{F}}(X)$ is the space of functions generated by $\mathcal{F}(X)$ under translations by integral vectors.

Denote by $S_{X}^{(i)}$ the subset of subspaces $\underline{r} \in S_{X}$ of dimension $i$. Define the spaces

$$
\mathcal{F}_{i}(X):=\bigcap_{\underline{t} \in S_{X}^{(i-1)}} \operatorname{ker} \nabla_{X \backslash \underline{t}} \cap \mathcal{F}(X) .
$$

Denote by $\tilde{\mathcal{F}}_{i}(X)$ the space of functions generated by $\mathcal{F}_{i}(X)$ under translations by integral vectors. Set $M_{\geq i}$ as the open set of points in $M_{X}$ with the property that the orbit has dimension $\geq i$.

Theorem 3 For each $s \geq i \geq 0$, the index multiplicity map ind $d_{m}$ gives an isomorphism between $K_{G}^{0}\left(T_{G}^{*} M_{\geq i}\right)$ and the space $\tilde{\mathcal{F}}_{i}(X)$.

Finally one has very explicit descriptions of $D M(X)$ and of the spaces $\tilde{\mathcal{F}}_{i}(X)$ which are built from various spaces $D M\left(X_{i}\right)$.

We then investigate the formulas allowing us to pass from equivariant $K$-theory to equivariant cohomology.

In order to understand these explicit formulas in [18] we have introduced the infinitesimal index, called for short "infdex", a map from the equivariant cohomology, with compact supports, of the zeroes of the moment map to distributions on $\mathfrak{g}^{*}$.

We have proved several properties for this map which, at least in the case of the space $T_{G}^{*} M$, in principle allow us to reduce the computations to the case in which $G$ is a torus and the manifold is a complex linear representation $M_{X}$ of $G$. Where $X$ is a list of characters as before.

The equivariant cohomology of the open sets $M_{X, \geq i}$ can be computed from the structure of the algebra $S\left[\mathfrak{g}^{*}\right]\left[\left(\prod_{a \in X} a\right)^{-1}\right]$ as a module over the Weyl algebra studied in [17].

The equivariant cohomology with compact supports $H_{G, c}^{*}\left(T_{G}^{*} M_{X}^{\text {fin }}\right)$ of $T_{G}^{*} M_{X}^{\text {fin }}$ is isomorphic, as a $S\left[\mathfrak{g}^{*}\right]$-module, to a remarkable finite dimensional space $D(X)$ of polynomial functions on $\mathfrak{g}^{*}$, where $S\left[\mathfrak{g}^{*}\right]$ acts by differentiation. The space $D(X)$ is the continuous analogue of $D M(X)$ and it is defined as the space of solutions of a set of linear partial differential equations combinatorially associated to $X$ and has been of importance in approximation theory (see for example $[13,14]$ ). In fact in a natural way $D(X)$ is a component of $D M(X)_{\mathbb{C}}$ and it coincides with $D M(X)_{\mathbb{C}}$ in the special case of $X$ unimodular 7.2. 
At this point the notion of infinitesimal index comes into play. One can show, Theorem 34, that the infinitesimal index gives an isomorphism between $H_{G, c}^{*}\left(T_{G}^{*} M_{X}^{f i n}\right)$ and $D(X)$. After this one shows that, for each $i$, the infinitesimal index establishes an isomorphism between $H_{G, c}^{*}\left(T_{G}^{*} M_{X, \geq i}\right)$ and a space of splines $\tilde{\mathcal{G}}_{i}(X)$, introduced in [19] and analogous to the arithmetic spaces $\tilde{\mathcal{F}}_{i}(X)$, and generalizing $D(X)$.

Then Theorem 37 is a deconvolution formula which allows us to compute the function $\operatorname{ind}_{m}(A)$ in function of the distribution infdex $(\operatorname{ch}(\Sigma))$ by applying to it a Todd operator.

In order to develop this formula one needs results obtained by Dahmen-Micchelli in the purely combinatorial context of the semi-discrete convolution with the Box spline.

In this paper we do not give details for the proofs which can be found in the papers quoted.

\section{Part 1. Polytopes and splines}

\section{Polytopes}

Polytopes play a special role in the Theory. A convex polytope can be defined as the convex envelop of finitely many points or by finitely many linear inequalities. We take this second point of view and study polytopes which vary into families.

\subsection{From a list of vectors}

We start from a real, sometimes integer $n \times m$ matrix $X$. We always think of $X:=$ $\left(a_{1}, \ldots, a_{m}\right)$ as a list of vectors in $V=\mathbb{R}^{n}$, its columns. We assume that 0 is NOT in the convex hull of its columns.

From $A$ we make several constructions, algebraic, combinatorial, analytic etc.

Many people have contributed to the Theory from various branches of mathematics. From numerical analysis: A.A. Akopyan; Ben-Artzi, Asher; C.K. Chui, C. De Boor, W. Dahmen, H. Diamond, N. Dyn, K. Höllig, C. Micchelli, Jia, Rong Qing, A. Ron, A.A. Saakyan.

From geometry and combinatorics: In fact a lot of work originated from the seminal paper of Khovanskiı̌, Pukhlikov, who interpret the counting formulas as Riemann-Roch formulas for toric varieties. We should also mention Orlik and Solomon on cohomology, Baldoni, Brion, Szenes, Vergne, Jeffrey and Kirwan, on partition functions, polytopes etc.

From enumerative combinatorics: A.I. Barvinok, Matthias Beck, Sinai Robins, Richard Stanley.

From $X$ we produce first a system of linear equations:

$$
\sum_{i=1}^{m} a_{i} x_{i}=b, \quad \text { or } \quad X x=b, \quad X:=\left(a_{1}, \ldots, a_{m}\right)
$$


The columns $a_{i}, b$ are vectors with $n$ coordinates $a_{j, i}, b_{j}, j=1, \ldots, n$.

As in Linear Programming Theory we deduce and want to study the, convex and bounded, variable polytopes:

$$
\Pi_{X}(b):=\left\{x \mid X x=b, x_{i} \geq 0, \forall i\right\}, \quad \Pi_{X}^{1}(b):=\left\{x \mid X x=b, 1 \geq x_{i} \geq 0, \forall i\right\} .
$$

The variable polytope $\Pi_{X}(b)$ is empty unless $b$ belongs to $C(X):=\left\{\sum_{i=1}^{m} x_{i} a_{i}\right.$, $\left.x_{i} \geq 0\right\}$, the cone of positive combinations of the $a_{i}$. The hypothesis that 0 is NOT in the convex hull of the $a_{i}$ implies that $C(X)$ is pointed, i.e. there is a linear function $\phi$ with $\phi\left(a_{i}\right)>0$ for all $i$ so that $\phi\left(a_{i}\right)>c>0$ / In other words $\phi$ is strictly positive on all non zero points of $C(X)$.

The property of being bounded is trivial for $\Pi_{X}^{1}(b)$ while for $\Pi_{X}(b)$ depends on the fact that

$$
\sum_{i} x_{i} a_{i}=b \Longrightarrow \sum_{i} x_{i} \phi\left(a_{i}\right)=\phi(b) \Longrightarrow \sum_{i} x_{i}<\phi(b) c^{-1} .
$$

The polytopes $\Pi_{X}^{1}(b)$ are sections of a hypercube,
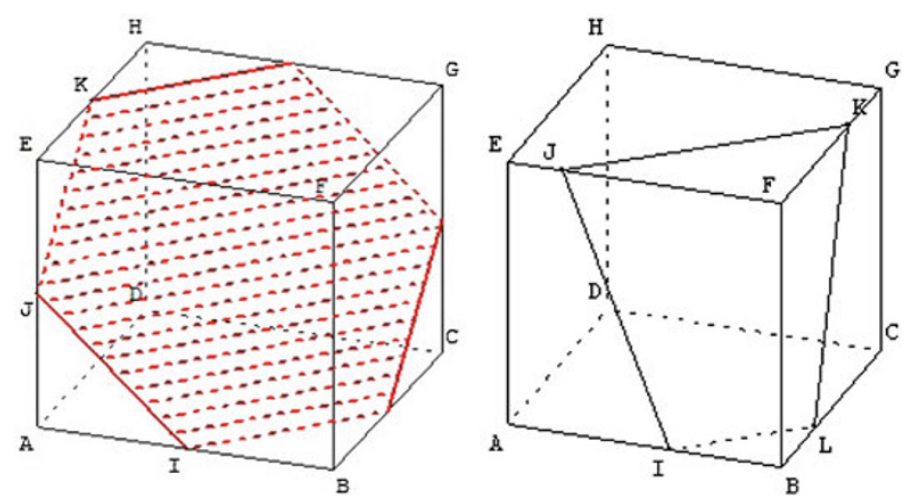

The object of study are two basic functions

- Set $T_{X}(x), B_{X}(x)$ to be the volume of $\Pi_{X}(x), \Pi_{X}^{1}(x)$.

- If $X, b$ have integer coordinates set $P_{X}(x)$ to be the number of solutions of the system in which the coordinates $x_{i}$ are non negative integers.

- In other words $P_{X}(x)$ is the number of integral points in the variable polytope $\Pi_{X}(x)$.

Up to a multiplicative normalization constant: $T_{X}(x)$ is the Multivariate-spline, $B_{X}(x)$ the Box-spline, $P_{X}(x)$ is called the partition function. We are interested in computing the three functions $T_{X}(x), B_{X}(x), P_{X}(x)$ and describe their qualitative properties.

We shall discuss applications of these functions to arithmetic, numerical analysis, Lie theory and Index Theory. 
An important example is when $X$ is the list of positive roots of a root system, e.g. $B_{2}$ :

$$
X=\left|\begin{array}{cccc}
-1 & 1 & 1 & 0 \\
1 & 0 & 1 & 1
\end{array}\right|
$$

The associated cone $C(X)$ has three big cells (cf. Sect. 2.9):

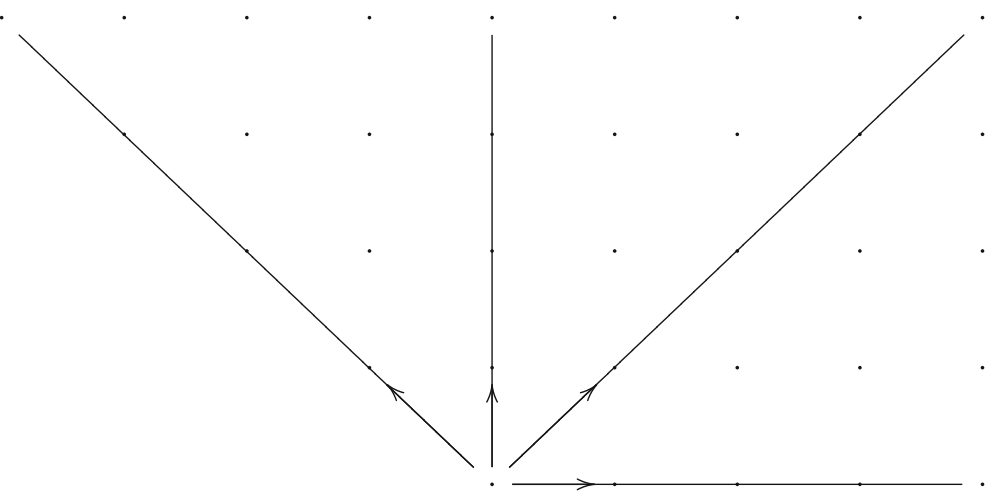

In the literature of numerical analysis the Box spline associated to the root system $B_{2}$ is called the Zwart-Powell or ZP element, $2 T_{X}$ is 0 outside the cone and on the three cells (Fig. 1):

The computation of the box-spline has some geometric, combinatorial and algebraic flavor. It appears as a piecewise polynomial function on a compact polyhedron. From simple data we get soon a complicated picture! (Fig. 2).

\subsection{The partition function}

When $X, b$ have integer entries, it is natural to think of an expression like: $b=$ $t_{1} a_{1}+\cdots+t_{m} a_{m}$, with $t_{i}$ not negative integers, as a partition of $b$ with the vectors $a_{i}$,

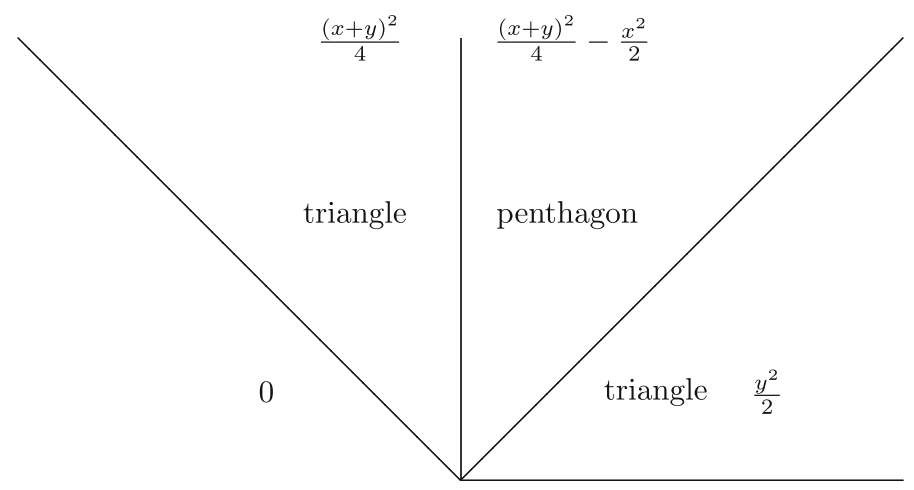

Fig. 1 The area and the shape as $b=(x, y)$ varies in the cone 


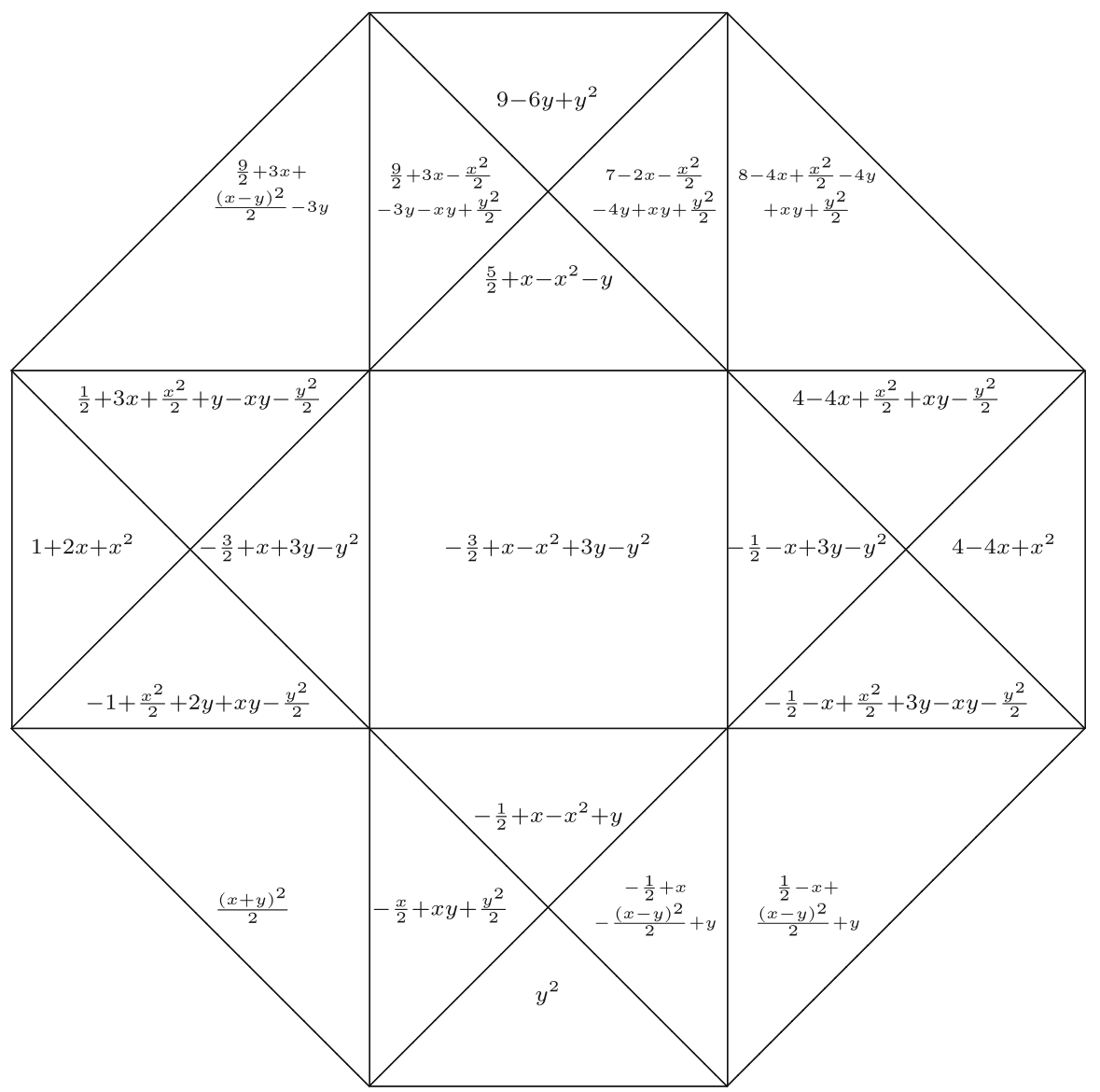

Fig. 2 The box spline $2 B_{X}$, ZP element

in $t_{1}+t_{2}+\cdots+t_{m}$ parts, hence the name partition function for the number $P_{X}(b)$, thought of as a function of the vector $b$.

Of special interest is the case when the $a_{i}$ are numbers. Given positive integers $\underline{h}:=\left(h_{1}, \ldots, h_{m}\right)$, the problem of counting the number of ways in which a positive integer $n$ can be written as a linear combination $\sum_{i=1}^{n} k_{i} h_{i}$, with the $k_{i}$ again positive integers, is a basic question in arithmetic. Denote by $P_{\underline{h}}(n)$ this number.

Example $2.3 m=2, n=1, X=\{2,3\}$. In how many ways can you write a number $b$ as: $b=2 x+3 y, \quad x, y \in \mathbb{N}$ ? The answer depends on the class of $n$ modulo 6

$$
\frac{n}{6}+1, \frac{n}{6}-\frac{1}{6}, \frac{n}{6}+\frac{2}{3}, \frac{n}{6}+\frac{1}{2}, \frac{n}{6}+\frac{1}{3}, \frac{n}{6}+\frac{1}{6}
$$

A function on a lattice $\Lambda$ which is a polynomial on each coset of some sublattice $M$ of finite index is called a quasi-polynomial. 
0

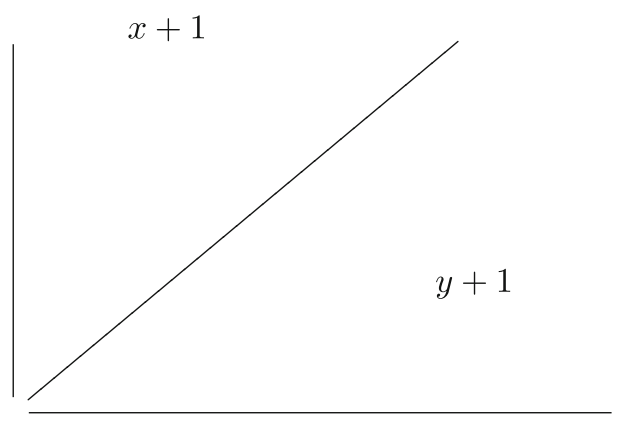

0

Fig. 3 The partition function for $A_{2}$

For the root system $A_{2}$ we may take $X=\left|\begin{array}{lll}1 & 0 & 1 \\ 0 & 1 & 1\end{array}\right|$. The corresponding partition function $P_{X}$ is piecewise polynomial with top degree coinciding with $T_{X}$ (Fig. 3).

Example 2.4 In Lie theory the Kostant partition function counts in how many ways can you decompose a weight as a sum of positive roots.

This is used in many computations.

2.4.1. The case of integers. The number $P_{h}(n)$ of ways in which $n$ is written as combination of $m$ numbers $h_{i}$ is already studied by Euler and called by Sylvester a denumerant. The first results on denumerants are due to Cayley and Sylvester, who proved that such a denumerant is a polynomial in $n$ of degree $m-1$ plus a periodic polynomial of lower degree called an undulant. A different approach is also developed by Bell in (1943). One starts with

Lemma 2.5 $P_{\underline{h}}(n)$ is the coefficient of $x^{n}$ in the power series expansion of:

$$
S_{\underline{h}}(x):=\prod_{i=1}^{m} \frac{1}{1-x^{h_{i}}}=\sum_{n=0}^{\infty} P_{\underline{h}}(n) x^{n} .
$$

In order to compute for a given $n \geq 0$, the coefficient $P_{\underline{h}}(n)$ of $x^{n}$ in $S_{\underline{h}}(x)$ we can use two essentially equivalent strategies:

1. Develop $S_{\underline{h}}(x)$ in partial fractions.

2. Compute the residue

$$
\frac{1}{2 \pi i} \oint \frac{x^{-n-1}}{\prod_{i} 1-x^{h_{i}}} d x
$$

around 0 . 
In both cases first we must expand in a suitable way the function $S_{\underline{h}}(x)$. Given $k$ let us denote by $\zeta_{k}:=e^{\frac{2 \pi i}{k}}$, a primitive $k$ th root of 1 ; we can write

$$
S_{\underline{h}}(x)=\prod_{i=1}^{m} \prod_{j=0}^{h_{i}-1} \frac{1}{1-\zeta_{h_{i}}^{j} x} .
$$

Let $\mu$ be the least common multiple of the numbers $h_{i}$, and write $\mu=h_{i} k_{i}$. If $\zeta=e^{2 \pi i / \mu}$, we have $\zeta_{h_{i}}=\zeta^{k_{i}}$ therefore we have

\section{Lemma 2.6}

$$
S_{\underline{h}}(x)=\prod_{i} \frac{1}{1-x^{h_{i}}}=\prod_{i=1}^{m} \prod_{j=0}^{h_{i}-1} \frac{1}{1-\zeta^{k_{i} j} x}=\prod_{\ell=0}^{\mu-1} \frac{1}{\left(1-\zeta^{\ell} x\right)^{b_{\ell}}}
$$

where the integer $b_{\ell}$ is the number of $k_{i}$ which are divisors of $\ell$.

In particular the function $x^{-n-1} S_{h}(x), n \geq 0$ has poles at 0 and at the $\mu$ th roots of 1 (but not at $\infty$ ).

The classical method starts from the fact that there exist numbers $c_{i}$ for which:

$$
\prod_{i=0}^{\mu-1} \frac{1}{\left(1-\zeta^{i} x\right)^{b_{i}}}=\sum_{i=0}^{\mu-1} \sum_{k=1}^{b_{i}} \frac{c_{i, k}}{\left(1-\zeta^{i} x\right)^{k}}
$$

We then use the simple formula:

$$
\frac{1}{(1-t)^{k}}=\sum_{h=0}^{\infty}\left(\begin{array}{c}
k-1+h \\
h
\end{array}\right) t^{h}
$$

to get:

$$
\prod_{i=0}^{\mu-1} \frac{1}{\left(1-\zeta^{i} x\right)^{b_{i}}}=\sum_{i=0}^{\mu-1} \sum_{k=1}^{b_{i}} c_{i, k}\left[\sum_{h=0}^{\infty}\left(\begin{array}{c}
k-1+h \\
h
\end{array}\right)\left(\zeta^{i} x\right)^{h}\right] .
$$

We have thus obtained a formula for the coefficient

$$
P_{\underline{h}}(n)=\sum_{i=0}^{\mu-1} \zeta^{i n}\left[\sum_{k=1}^{b_{i}} c_{i, k}\left(\begin{array}{c}
k-1+n \\
n
\end{array}\right)\right] .
$$

Let us remark now that

$$
\left(\begin{array}{c}
k-1+n \\
n
\end{array}\right)=\frac{(n+1)(n+2) \cdots(n+k-1)}{(k-1) !}
$$


is a polynomial of degree $k-1$ in $n$, while the numbers $\zeta^{i n}$ depend only from the coset of $n$ modulo $\mu$. Given an $0 \leq a<\mu$ and restricting us to the numbers $n=\mu k+a$, we have:

Theorem 4 The function $P_{h}(m k+a)$ is a polynomial in the variable $k$ of degree $\leq \max \left(b_{i}\right)$, that can be computed.

The fact that $P_{\underline{h}}(n)$ is a polynomial on every coset means that it is a quasi-polynomial or a periodic polynomial.

Second method: computation of residues

Here the strategy is the following: shift the computation of the residue to the remaining poles, taking advantage of the fact that the sum of residues at all the poles of a rational function is 0 .

From the theory of residues we have:

$$
\frac{1}{2 \pi i} \oint \prod_{i} \frac{x^{-n-1}}{1-x^{h_{i}}} d x=-\sum_{j=1}^{\mu-1} \frac{1}{2 \pi i} \oint_{C_{j}} \prod_{t=1}^{\mu} \frac{x^{-n-1}}{\left(1-\zeta^{t} x\right)^{b_{t}}} d x
$$

where $C_{j}$ is a small circle around $\zeta^{-j}$. In order to compute the term

$$
\frac{1}{2 \pi i} \oint_{C_{j}} \prod_{t=1}^{\mu} \frac{x^{-n-1}}{\left(1-\zeta^{t} x\right)^{b_{t}}} d x
$$

we perform the change of coordinates $x=w+\zeta^{-j}$ obtaining:

$$
\frac{1}{2 \pi i} \oint_{C_{j}-\zeta^{-j}} \prod_{t=1}^{\mu} \frac{\left(w+\zeta^{-j}\right)^{-n-1}}{\left(1-\zeta^{t-j}-\zeta^{t} w\right)^{b_{t}}} d w
$$

Finally we have that (6) equals:

$$
\begin{aligned}
& \frac{(-1)^{b_{j}}}{2 \pi i} \oint_{C_{j}-\zeta^{-j}} \zeta^{j\left(n+1-b_{j}\right)}\left(\sum_{k=0}^{\infty}(-1)^{k}\left(\begin{array}{c}
n+k \\
k
\end{array}\right) \zeta^{j k} w^{k}\right)\left(\sum_{h=0}^{\infty} a_{j, h} w^{h}\right) w^{-b_{j}} d w \\
& =(-1)^{b_{j}} \zeta^{j\left(n+1-b_{j}\right)} \sum_{k+h=b_{j}-1}(-1)^{k} \zeta^{j k}\left(\begin{array}{c}
n+k \\
k
\end{array}\right) a_{j, h} .
\end{aligned}
$$

Summing over $j$ we obtain an explicit formula for $P_{h}(n)$, again as a quasipolynomial.

Remark that, in order to develop these formulae it suffices to compute a finite number of coefficients $a_{j, h}$.

In these formulae roots of unity appear while the final partition functions are clearly integer valued. An algorithmic problem remains. We need to know how to manipulate these expressions involving roots on 1 . 
This is an elementary but computationally very complex problem on cyclotomic polynomials. Set $\zeta_{k}:=e^{\frac{2 \pi i}{k}}$ a primitive root of 1 . Define:

$$
\psi(k, x):=\operatorname{tr}_{\mathbb{Q}}^{\mathbb{Q}\left(\zeta_{k}\right)}\left(\zeta_{k}^{x}\right), \quad x \in \mathbb{Z} .
$$

This is a periodic integral valued function, computable through the Möbius function and the Euler $\phi$ function. One can use these functions in order to find explicit formulas for the partition function. For instance:

Example $2.7(X=2,3,4,4,6,9,10,40)$.

- The formula is over all the roots of 1 of order a divisor of an element in $X$.

- The 10 Galois orbits of primitive roots correspond to the divisors 2, 3, 4, 5, 6, 8, 9, $10,20,40$.

- The quasi-polynomial associated to the partition function, decomposes according to the divisors.

This has been computed with Mathematica and it is:

$$
\begin{aligned}
& \frac{1262093963}{2985984000}+\frac{252100403 x}{2508226560}+\frac{81263 x^{2}}{2457600}+\frac{3303691 x^{3}}{995328000}+\frac{27443 x^{4}}{199065600}+\frac{1639 x^{5}}{597196800}+\frac{13 x^{6}}{497664000}+ \\
& \frac{x^{7}}{10450944000}+\frac{1173757 \psi(2, x)}{4096000}+\frac{483129 x \psi(2, x)}{4096000}+\frac{26273 x^{2} \psi(2, x)}{2457600}+\frac{1621 x^{3} \psi(2, x)}{4423680}+\frac{13 x^{4} \psi(2, x)}{2457600}+ \\
& \frac{x^{5} \psi(2, x)}{36864000}+\frac{1009 \psi(3, x)}{5832}-\frac{11 x \psi(3, x)}{4374}-\frac{x^{2} \psi(3, x)}{8748}-\frac{149 \psi(3,1+x)}{972}-\frac{13 x \psi(3,1+x)}{729}- \\
& \frac{x^{2} \psi(3,1+x)}{4374}+\frac{81 \psi(4, x)}{1280}+\frac{39 x \psi(4, x)}{10240}+\frac{x^{2} \psi(4, x)}{20480}+\frac{117 \psi(4,1+x)}{10240}+\frac{3 x \psi(4,1+x)}{10240}+\frac{89 \psi(5, x)}{10000}+ \\
& \frac{x \psi(5, x)}{5000}+\frac{123 \psi(5,1+x)}{10000}+\frac{3 x \psi(5,1+x)}{10000}+\frac{111 \psi(5,2+x)}{10000}+\frac{3 x \psi(5,2+x)}{10000}+\frac{67 \psi(5,3+x)}{10000}+ \\
& \frac{x \psi(5,3+x)}{5000}-\frac{\psi(6, x)}{648}+\frac{\psi(6,1+x)}{324}-\frac{\psi(8,1+x)}{640}-\frac{2 \psi(9, x)}{81}+\frac{\psi(9,2+x)}{81}-\frac{\psi(9,3+x)}{81}-\frac{\psi(9,4+x)}{81}+ \\
& \frac{13 \psi(10, x)}{10000}+\frac{169 \psi(10,1+x)}{10000}+\frac{x \psi(10,1+x)}{2000}-\frac{221 \psi(10,2+x)}{10000}-\frac{x \psi(10,2+x)}{2000}+\frac{13 \psi(10,3+x)}{10000}+ \\
& \frac{\psi(20,1+x)}{200}-\frac{\psi(20,2+x)}{200}+\frac{\psi(20,3+x)}{400}+\frac{\psi(20,4+x)}{400}+\frac{\psi(20,5+x)}{400}-\frac{\psi(20,6+x)}{200}+\frac{\psi(20,7+x)}{200}- \\
& \frac{3 \psi(40,3+x)}{80}-\frac{\psi(40,4+x)}{80}-\frac{\psi(40,5+x)}{80}-\frac{\psi(40,6+x)}{20}-\frac{\psi(40,7+x)}{40}-\frac{\psi(40,9+x)}{20}-\frac{\psi(40,11+x)}{40}- \\
& \frac{\psi(40,12+x)}{20}-\frac{\psi(40,13+x)}{80}-\frac{\psi(40,14+x)}{80}-\frac{3 \psi(40,15+x)}{80}
\end{aligned}
$$

\subsection{Main objects associated to $X$}

We now go back to the general case of a list $X=\left\{a_{1}, \ldots, a_{m}\right\}$ of vectors. We think of the elements $a_{i}$ as linear equations defining hyperplanes in the dual space $U=V^{*}$. This is a central hyperplane arrangement.

One way to study this arrangement is to study the algebra of rational functions which have poles only on this arrangement. That is we consider the algebra

$$
R_{X}:=S[V]\left[\prod_{i=1}^{m} a_{i}^{-1}\right]
$$

of polynomials $S[V]$ localized at $\prod_{i=1}^{m} a_{i}$. 
The algebra $R_{X}$ consists of those rational functions which have at the denominator a product of powers of the linear forms $a_{i}$. It is clearly a module under the Weyl algebra of differential operators with polynomial coefficients and we analyze in depth the module structure.

When the coordinates of the elements $a_{i}=\left(a_{i, 1}, \ldots, a_{i, s}\right)$ are integers we take instead a multiplicative point of view and think of $a_{i}$ as a character $\prod_{j=1}^{s} x_{j}^{a_{i, j}}$.

This defines a subgroup of the torus $\left(\mathbb{C}^{*}\right)^{s}$ the points in which the character is 1 or where $1-\prod_{j=1}^{s} x_{j}^{a_{i, j}}$ vanishes.

This is call a central toric arrangement (by the exponential map this corresponds in fact to a periodic hyperplane arrangement).

One way to study this arrangement is to study the algebra of Laurent polynomials $S\left[x_{1}^{ \pm 1}, \ldots, x_{s}^{ \pm 1}\right]$ localized at $\prod_{i=1}^{m}\left(1-\prod_{j=1}^{s} x_{j}^{a_{i, j}}\right)$ that is the algebra

$$
S\left[x_{1}^{ \pm 1}, \ldots, x_{s}^{ \pm 1}\right]\left[\prod_{i=1}^{m}\left(1-\prod_{j=1}^{s} x_{j}^{a_{i, j}}\right)^{-1}\right] \text {. }
$$

We study also this as a module over the algebra of differential operators with coefficients Laurent polynomials.

\subsection{The theorems}

There are several general formulas to compute the previous functions which are obtained by a mixture of techniques.

A main geometric notion that plays a role is that of big cells. Assume that $X$ spans $V$.

- The singular points $C^{\text {sing }}(X)$ are the points in the cone $C(X)$ lying in some cone $C(Y)$ for any sublist $Y$ of $X$ which does NOT span the ambient space.

- The other points are called regular.

- A big cell is a connected component of the set or regular points.

In other words, take all the hyperplanes $H$ spanned by sublists of $X$ and then the cones $C(H \cap X)$. The union of all these cones forms the set of singular vectors.

It is easy to see that the big cells are convex polyhedra.

Take all bases $\underline{b}$ extracted from $X$, for each basis consider the cone $C(\underline{b})$ generated by $\underline{b}$. Its boundary is made of singular points.

A standard fact of polyhedra is that $C(X)=\cup_{\underline{b}} C(\underline{b})$ so if a point $p \in C(X)$ is regular it lies either in the interior $\stackrel{\circ}{C}(\underline{b})$ of outside each $C(\underline{b})$. It follows that the big cell $\mathfrak{c}$ in which $p$ lies is the intersection of all the $\stackrel{\circ}{C}(\underline{b})$ containing it: $\mathfrak{c}=\cap_{\underline{b} \mid p \in \stackrel{\circ}{C}(\underline{b})} \stackrel{\circ}{C}(\underline{b})$.

Example 2.10 The positive roots of type $A_{3}$ are $\alpha_{1}, \alpha_{2}, \alpha_{3}, \alpha_{1}+\alpha_{2}, \alpha_{2}+\alpha_{3}, \alpha_{1}+$ $\alpha_{2}+\alpha_{3}$.

We want to decompose the cone $C(X)$ into big cells and see its singular and regular points. We do this on a transversal section (Fig. 4). 


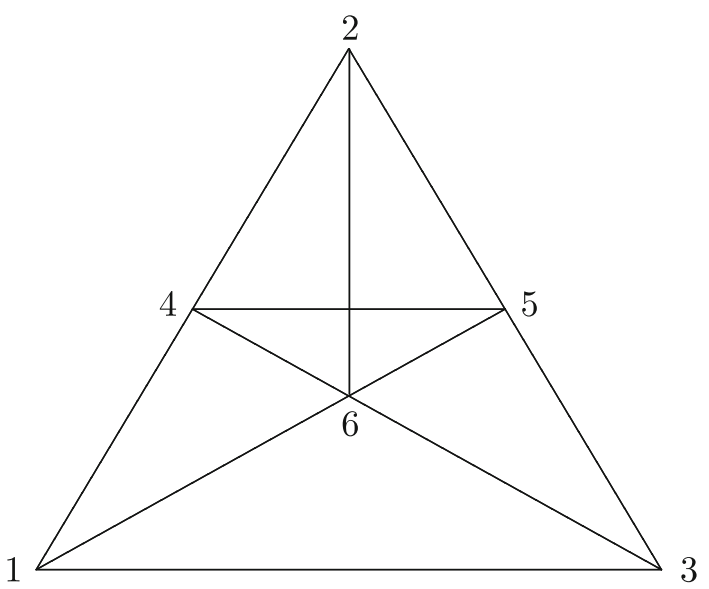

Fig. 4 The 7 big cells for type $A_{3}$ in cross section

2.10.1. Towards the Jeffrey-Kirwan formula. Assume $X$ spans $\mathbb{R}^{n}$. Let $m(X)$ denote the minimum number of columns that one can remove from $X$ so that the remaining columns do not span $\mathbb{R}^{n}$.

(i) $T_{X}(x)$ has support on the cone $C(X)$.

(ii) $T_{X}(x)$ is of class $m(X)-2$.

(iii) $T_{X}(x)$ coincides with a homogeneous polynomial of degree $m-n$ on each big cell.

In order to compute $T_{X}(x)$ we need to

(i) Determine the decomposition of $C(X)$ into cells.

(ii) Compute on each big cell the homogeneous polynomial of degree $m-n$ coinciding with $T_{X}(x)$.

One can find explicit polynomials $p_{b, X}(x)$, indexed by a combinatorial object called unbroken bases (see Sect. 3.1) and characterized by certain explicit differential equations so that, given a point $x$ in the closure of a big cell $\mathfrak{c}$ we have the (cf. Sect. 9.6.1)

$$
\text { Jeffry-Kirwan residue formula } T_{X}(x)=\sum_{\underline{b} \mid \mathfrak{c} \subset C(\underline{b})}|\operatorname{det}(\underline{b})|^{-1} p_{\underline{b}, X}(-x) .
$$

From $T_{X}$ one computes $B_{X}$. For a given subset $S$ of $X$ define $a_{S}:=\sum_{a \in S} a$; the basic formula is:

$$
B_{X}(x)=\sum_{S \subset X}(-1)^{|S|} T_{X}\left(x-a_{S}\right)
$$

So $T_{X}$ is the fundamental object.

Notice that the local pieces of $B_{X}$ are no more homogeneous polynomials. 


\section{Combinatorics}

\subsection{Unbroken bases}

We now explain some ideas relating unbroken bases with cells.

From the theory of matroids to cells.

Let $\underline{c}:=a_{i_{1}}, \ldots, a_{i_{k}} \in X, i_{1}<i_{2} \cdots<i_{k}$, be a sublist of linearly independent elements.

Definition 3.2 We say that $a_{i}$ breaks $\underline{c}$ if there is an index $1 \leq e \leq k$ such that:

- $i \leq i_{e}$.

- $a_{i}$ is linearly dependent on $a_{i_{e}}, \ldots, a_{i_{k}}$.

Example 3.3 Take as $X$ the list of positive roots for type $A_{3}$.

$$
X=\left\{\alpha_{1}, \alpha_{2}, \alpha_{3}, \alpha_{1}+\alpha_{2}, \alpha_{2}+\alpha_{3}, \alpha_{1}+\alpha_{2}+\alpha_{3}\right\}
$$

We have 16 bases 10 broken and 6 unbroken, all contain necessarily $\alpha_{1}$ :

$$
\begin{array}{lll}
\alpha_{1}, \alpha_{2}, \alpha_{3}, \alpha_{1}+\alpha_{2}, & \alpha_{2}+\alpha_{3}, \alpha_{1}+\alpha_{2}+\alpha_{3} . \\
\alpha_{1}, & \alpha_{2}, \alpha_{3}, \alpha_{1}+\alpha_{2}, \alpha_{2}+\alpha_{3}, \alpha_{1}+\alpha_{2}+\alpha_{3} . \\
\alpha_{1}, & \alpha_{2}, \alpha_{3}, \alpha_{1}+\alpha_{2}, \alpha_{2}+\alpha_{3}, \alpha_{1}+\alpha_{2}+\alpha_{3} . \\
\alpha_{1}, \alpha_{2}, \alpha_{3}, \alpha_{1}+\alpha_{2}, & \alpha_{2}+\alpha_{3}, \alpha_{1}+\alpha_{2}+\alpha_{3} . \\
\alpha_{1}, \alpha_{2}, \alpha_{3}, \alpha_{1}+\alpha_{2}, & \alpha_{2}+\alpha_{3}, \alpha_{1}+\alpha_{2}+\alpha_{3} . \\
\alpha_{1}, \alpha_{2}, \alpha_{3}, \alpha_{1}+\alpha_{2}, \alpha_{2}+\alpha_{3}, \alpha_{1}+\alpha_{2}+\alpha_{3} .
\end{array}
$$

The overlapping theorem states that, by overlapping the cones generated by the unbroken bases one obtains the entire decomposition into big cells!!

PROBLEM: Describe the previous pictures for root systems. For type $A_{n}$ the unbroken bases are known and can be indexed by certain binary graphs or by permutations of $n$ elements. The decomposition into cells is unknown.

3.3.1. The zonotope. The box spline $B_{X}(x)$ is supported in the compact polytope: called the zonotope:

$$
B(X):=\left\{\sum_{i=1}^{m} t_{i} a_{i}\right\}, 0 \leq t_{i} \leq 1, \forall i
$$

When $X$ is the set of positive roots of a root system and $\rho:=1 / 2 \sum_{a \in X} a$ we have:

Theorem $5 B(X)-\rho$ is the convex envelop of the orbit of $\rho$ under the action of the Weyl group. 
The zonotopes associated to the root system $A_{3} B_{3}$
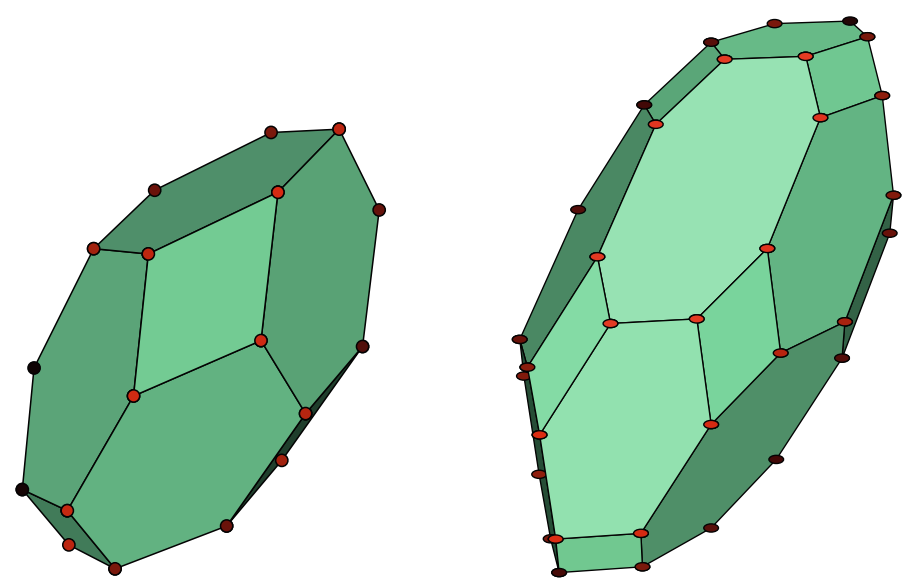

We now want to describe the faces of $B(X)$. Consider a sublist $A$ of $X$ spanning a hyperplane $H_{0}$ and such that $A=H_{0} \cap X$.

Take a linear equation $\phi_{H_{0}}$ for $H_{0}$ and set

$$
B=\left\{a \in X \mid\left\langle\phi_{H_{0}} \mid a\right\rangle>0\right\}, \quad C=\left\{a \in X \mid\left\langle\phi_{H_{0}} \mid a\right\rangle<0\right\} .
$$

Let $A, B$ be two disjoint sublists of $X$ such that $A$ does not span $V$ and $B(A)$ its associated zonotope. Let

$$
B(X)_{A, B}:=\sum_{a_{i} \in A} t_{i} a_{i}+\sum_{b \in B} b, 0 \leq t_{i} \leq 1=B(A)+\lambda_{B}
$$

with $\lambda_{B}:=\sum_{b \in B} b$.

Proposition 3.4 $\bullet B(X)_{A, B}$ and $B(X)_{A, C}$ are exactly the two codimension one faces in $B(X)$ parallel to $H_{0}$.

- All codimension 1 faces occur in this way.

Let $\mathcal{H}_{X}$ be the real hyperplane arrangement defined, in dual space, by the vectors of $X$ thought of as linear equations.

Theorem 6 - There is an order reversing 1-1 correspondence between faces of $B(X)$ and faces of $\mathcal{H}_{X}$.

- To a face $G$ of the hyperplane arrangement we associate the set of points in $B(X)$ where any element $f \in G$ takes its maximum value.

- This is the face $B(X)_{A, B}$ where $A:=\{x \in X \mid\langle x \mid f\rangle=0\}$ and $B:=\{x \in$ $X \mid\langle x \mid f\rangle>0\}$.

The zonotope $B(X)$ has a nice combinatorial structure, proved by Shephard, it can be paved by a set of parallelepipeds indexed by all the bases which one can extract from $A$ ! 
Example 3.5 In the next example

$$
X=\left|\begin{array}{cccccc}
1 & 0 & 1 & -1 & 2 & 1 \\
0 & 1 & 1 & 1 & 1 & 2
\end{array}\right|
$$

we have 15 bases and 15 parallelograms.

$$
X=\left|\begin{array}{cccccc}
1 & 0 & 1 & -1 & 2 & 1 \\
0 & 1 & 1 & 1 & 1 & 2
\end{array}\right|
$$

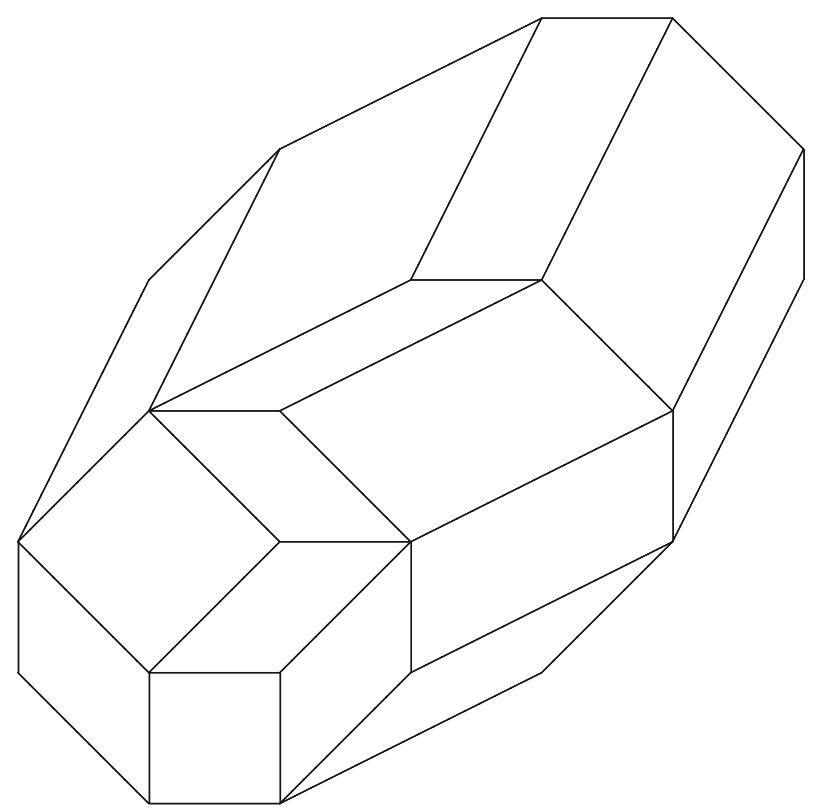

This can be constructed stepwise adding a vector at a time.

$$
=\left|\begin{array}{cccccc}
1 & 0 & 1 & -1 & 2 & 1 \\
0 & 1 & 1 & 1 & 1 & 2
\end{array}\right|
$$

\section{START WITH}

$$
X=\left|\begin{array}{ll}
1 & 0 \\
0 & 1
\end{array}\right|
$$

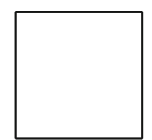


Then $\left|\begin{array}{lll}1 & 0 & 1 \\ 0 & 1 & 1\end{array}\right|$

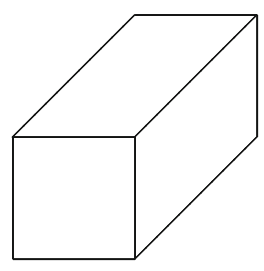

$\left|\begin{array}{cccc}1 & 0 & 1 & -1 \\ 0 & 1 & 1 & 1\end{array}\right|$

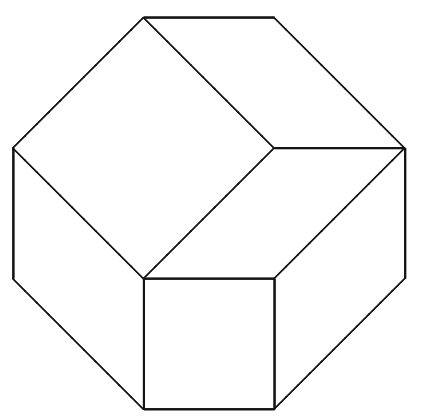

and so on.

\section{Splines}

By Fubini's Theorem the volume $V_{X}(x)$, of the variable polytope $\Pi_{X}(x)$, is $\sqrt{\operatorname{det} X X^{t}} T_{X}(x)$. The function $T_{X}(x)$ called multivariate spline is characterized by the formula:

$$
\int_{\mathbb{R}^{n}} f(x) T_{X}(x) d x=\int_{\mathbb{R}_{+}^{m}} f\left(\sum_{i=1}^{m} t_{i} a_{i}\right) d t,
$$

where $f(x)$ is any continuous function with compact support.

In general $T_{X}(x)$ is a tempered distribution, supported on the cone $C(X)$, only when $X$ has maximal rank $T_{X}(x)$ is a function.

While the function $T_{X}(x)$ is the basic object, the more interesting object for numerical analysis is the box spline, that is the function $B_{X}(x)$ characterized by the formula: 


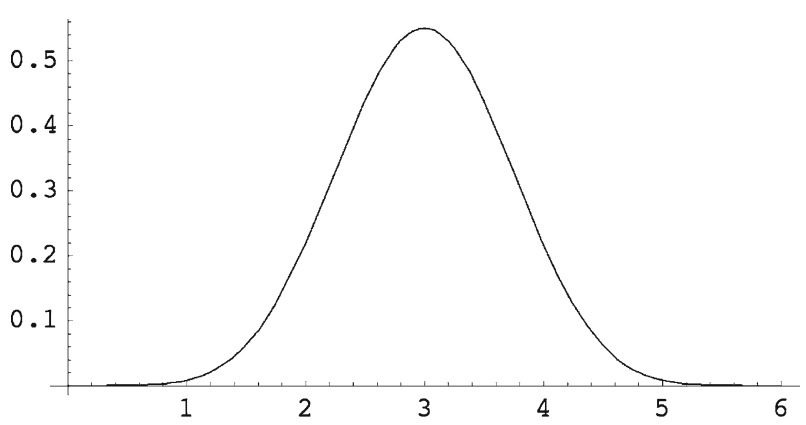

Fig. 5 The function $b_{5}(x)$

$$
\int_{\mathbb{R}^{n}} f(x) B_{X}(x) d x=\int_{[0,1]^{m}} f\left(\sum_{i=1}^{m} t_{i} a_{i}\right) d t
$$

where $f(x)$ is any continuous function.

Example 4.1 The $b_{m}$-spline of Schoenberg $X=\{1,1, \ldots, 1\}, m+1$-times is of class $C^{m-1}$, it is supported in the interval $[0, m+1]$, and given by the formula:

$$
b_{m}(x):=\sum_{i=0}^{k}(-1)^{i}\left(\begin{array}{c}
m+1 \\
i
\end{array}\right) \frac{(x-i)^{m}}{m !}, \forall x \in[k, k+1] .
$$

4.1.1. Properties of box-splines.

$$
\int_{\mathbb{R}^{n}} B_{X}(x) d x=1, \quad B_{[X, v]}(x)=\int_{0}^{1} B_{X}(x-t v) d t
$$

In the case of integral vectors, we have that the translates $B_{X}(x-\lambda)$, as $\lambda$ runs over the integral vectors form a partition of 1 (Fig. 5).

\subsection{Approximation theory}

The box spline, when the $a_{i}$ are integral vectors, can be effectively used in the finite element method to approximate functions.

We define the Cardinal spline space as in [31].

$$
\mathcal{S}_{X}:=\left\{\sum_{i \in \mathbb{Z}^{s}} B_{X}(x-i) a_{i}\right\}
$$

The function $a_{i}$ on $\mathbb{Z}^{s}$ is called a mesh function.

Define $D(X)$ to be the space of polynomials contained in $\mathcal{S}_{X}$. 
For a function $f$ we have the semi-discrete convolution

$$
f \mapsto B_{X} *^{\prime} f:=\sum_{i \in \mathbb{Z}^{s}} B_{X}(x-i) f(i)
$$

The theory of Dahmen-Micchelli consists of several Theorems.

The basic results are:

- Semi-discrete convolution maps $D(X)$ into $D(X)$.

- The dimension of $D(X)$ equals the number of bases which one can extract from $X$.

- $D(X)$ contains all polynomials of degree $<m(X)(m(X)$ is the minimum length of a cocircuit).

- $D(X)$ is the space of solutions of the differential equations $D_{Y} f=0$ as

(i) $Y$ runs over the cocircuits of $X$.

(ii) $D_{Y}:=\prod_{a \in Y} D_{a}$

(iii) $D_{a}$ is the directional derivative relative to $a$.

Example 4.3 The cardinal spline space associated to $b_{m}(x)$ is the space of all functions of class $C^{m-1}$ which, on each interval $[n, n+1], n \in \mathbb{Z}$ coincide with a polynomial of degree $\leq m$.

\section{Algebra}

How to compute $T_{X}$ ? or the partition function $P_{X}$ ? We use the Fourier-Laplace transform $L f(u):=\int_{V} e^{-\langle u \mid v\rangle} f(v) d v$ which will change the analytic problem to one in algebra.

In coordinates $u=\left(y_{1}, \ldots, y_{s}\right), v=\left(x_{1}, \ldots, x_{s}\right)$ we have

$$
L f\left(y_{1}, \ldots, y_{s}\right):=\int_{\mathbb{R}^{s}} e^{-\sum_{i=1}^{s} y_{i} x_{i}} f\left(x_{1}, \ldots, x_{s}\right) d x_{1} \ldots d x_{s}
$$

5.0.1. Basic properties. Given $p \in U, w \in V$, write $p, D_{w}$ for the linear function $\langle p \mid v\rangle$ and the directional derivative on $V$. We then have:

$$
\begin{aligned}
& L\left(D_{w} f\right)(u)=w L f(u), L(p f)(u)=-D_{p} L f(u), L\left(e^{p} f\right)(u)=L f(u-p), \\
& L(f(v+w))(u)=e^{w} L f(u) .
\end{aligned}
$$

An easy computation gives the Laplace transforms:

$$
L B_{X}=\prod_{a \in X} \frac{1-e^{-a}}{a}, \quad L T_{X}=\prod_{a \in X} \frac{1}{a}
$$


We need to rewrite $L T_{X}=\prod_{a \in X} a^{-1}$, for this we need to develop a theory of partial fractions in several variables, in this case for the algebra

$$
S[V]\left[\prod_{a \in X} a^{-1}\right] .
$$

We do this using non commutative algebra.

Set $W(V), W(U)$ be the two Weyl algebras of differential operators with polynomial coefficients on $V$ and $U$. Both algebras are generated by $V \oplus U$ with the same relations so there is an algebraic Fourier isomorphism between these two algebras, so any $W(V)$ module $M$ becomes a $W(U)$ module, denoted by $\hat{M}$.

5.0.2. D-modules in Fourier duality. The Laplace transform gives rise, using the previous construction, to an isomorphism of two modules under the two algebras $W(V), W(U)$.

1. The $D$-module $\mathcal{D}_{X}:=W(V) T_{X}$ generated, in the space of tempered distributions, by $T_{X}$ under the action of the algebra $W(V)$ of differential operators on $V$ with polynomial coefficients.

2. The algebra $R_{X}:=S[V]\left[\prod_{a \in X} a^{-1}\right]=W(U) d_{X}^{-1}$ obtained from the polynomials on $U$ by inverting the element $d_{X}:=\prod_{a \in X} a$. The algebra $R_{X}$ is the coordinate ring of the open set $\mathcal{A}_{X}$ complement of the union of the hyperplanes of $U$ of equations $a=0, a \in X$. It is a cyclic module under $W(U)$ generated by $d_{X}^{-1}$.

It is well known that, once we invert an element in a polynomial algebra, we get a holonomic module over the algebra of differential operators (cf. [12]).

In particular $R_{X}$ is holonomic, cyclic and it has a finite composition series. In order to understand this composition series we observe that for each subspace $W$ of $U$ we have an irreducible module $N_{W}$ (over $W(U)$ ) generated by the $\delta$ function of $W\left(f \mapsto \int_{W} f(w) d w\right)$.

To be explicit, take coordinates $x_{1}, \ldots, x_{n} \in V$ so that $W=\left\{x_{1}=x_{2}=\cdots=\right.$ $\left.x_{k}=0\right\} . N_{W}$ is generated by an element $\delta_{W}$ satisfying: $x_{i} \delta_{W}=0, i \leq k, \frac{\partial}{\partial x_{i}} \delta, i>k$. $N_{W}$ is free of rank 1 generated by $\delta_{W}$ over: $\mathbb{C}\left[x_{1}, x_{2}, \ldots, x_{k}, \frac{\partial}{\partial x_{k+1}}, \ldots, \frac{\partial}{\partial x_{n}}\right]$.

In particular the composition factors of $R_{X}$ are of the form $N_{W}$ as $W$ runs over the subspaces of the hyperplane arrangement, in $U$, given by the equations $a_{i}=0, a_{i} \in X$. In order to explain this we exhibit an explicit filtration.

\subsubsection{The filtration of $R_{X}$ by polar order}

Definition 5.1 We denote by $R_{X, k}$ the span of all the fractions $f \prod_{a \in X} a^{-h_{a}}, h_{a} \geq 0$ for which the set of vectors $a$, with $h_{a}>0$, spans a space of dimension $\leq k$.

- $R_{X}$ is filtered by the $W(U)$-submodules $R_{X, k}$.

- We have $R_{X, s}=R_{X}$.

- For all $k$ we have that $R_{X, k} / R_{X, k-1}$ is semisimple.

- The isotypic components of $R_{X, k} / R_{X, k-1}$ are of type $N_{W}$ as $W$ runs over the subspaces of the arrangement of codimension $k$. 
- The space $R_{X, s} / R_{X, s-1}$ is a free module over

$$
S[U]=\mathbb{C}\left[\frac{\partial}{\partial x_{1}}, \ldots, \frac{\partial}{\partial x_{s}}\right]
$$

In fact a more precise statement holds. Denote by $R_{X}^{s}$ the linear span of all the fractions $\prod_{i=1}^{m} a_{i}^{-h_{i}}, h_{i} \geq 0$ so that the $a_{i}$ with $h_{i}>0$ span the space.

$R_{X}^{s}$ is a complement of $R_{X, s-1}$ in $R_{X, s}$ and it is a free module over

$$
S[U]=\mathbb{C}\left[\frac{\partial}{\partial x_{1}}, \ldots, \frac{\partial}{\partial x_{s}}\right]
$$

It is important to choose a basis.

Theorem 7 A basis for $R_{X}^{s} \equiv R_{X, s} / R_{X, s-1}$ over $S[U]$ is given by the elements $\prod_{a \in \underline{b}} a^{-1}$ as $\underline{b}$ runs over the set of unbroken bases.

Denote by $\mathcal{N B}$ the unbroken bases extracted from $X$.

We have in particular an expansion of $d_{X}^{-1}=\prod_{a \in X} a^{-1}$

$$
d_{X}^{-1}=\prod_{a \in X} a^{-1}=\sum_{\underline{b} \in \mathcal{N} \mathcal{B}} p_{\underline{b}} \prod_{a \in \underline{b}} a^{-1}, \quad p_{\underline{b}} \in S[U]=\mathbb{C}\left[\frac{\partial}{\partial x_{1}}, \ldots, \frac{\partial}{\partial x_{s}}\right]
$$

Example $5.2 A_{2}$ also called Courant element in the Theory of splines.

$$
\begin{gathered}
X=[x+y, x, y]=[x, y]\left|\begin{array}{lll}
1 & 0 & 1 \\
1 & 1 & 0
\end{array}\right| \\
\frac{1}{(x+y) x y}=\frac{1}{x(x+y)^{2}}+\frac{1}{y(x+y)^{2}}=-\frac{\partial}{\partial y}\left(\frac{1}{x(x+y)}\right)-\frac{\partial}{\partial x}\left(\frac{1}{y(x+y)}\right)
\end{gathered}
$$

Example $5.3 \quad B_{2}$ also called ZP element in the Theory of splines.

$$
\begin{gathered}
X=[x+y, x, y,-x+y]=[x, y]\left|\begin{array}{cccc}
1 & 0 & 1 & -1 \\
1 & 1 & 0 & 1
\end{array}\right| \\
\frac{1}{(x+y) x y(-x+y)}=\frac{1}{(x+y)^{3} x}+\frac{4}{(x+y)^{3}(-x+y)}-\frac{1}{(x+y)^{3} y} \\
=1 / 2\left[\frac{\partial^{2}}{\partial^{2} y}\left(\frac{1}{(x+y) x}\right)+\left(\frac{\partial}{\partial x}+\frac{\partial}{\partial y}\right)^{2}\left(\frac{1}{(x+y)(-x+y)}\right)-\frac{\partial^{2}}{\partial^{2} x}\left(\frac{1}{(x+y) y}\right)\right]
\end{gathered}
$$


We now need the basic inversion. Let $X=\left\{a_{1}, \ldots, a_{n}\right\}$ be a basis, $d:=$ $\left|\operatorname{det}\left(a_{1}, \ldots, a_{S}\right)\right|$ and $\chi_{C(X)}$ the characteristic function of the positive quadrant $C(X)$ generated by $X$.

$$
L\left(d^{-1} \chi_{C(X)}\right)=\prod_{i=1}^{n} a_{i}^{-1}
$$

We want to invert

$$
d_{X}^{-1}=\sum_{\underline{b} \in \mathcal{N} \mathcal{B}} p_{\underline{b}, X}\left(\frac{\partial}{\partial x_{1}}, \ldots, \frac{\partial}{\partial x_{s}}\right) \prod_{a \in \underline{b}} a^{-1}
$$

From the basic example and the properties we get

$$
L^{-1} d_{X}^{-1}=\sum_{\underline{b} \in \mathcal{N} \mathcal{B}} p_{\underline{b}, X}\left(-x_{1}, \ldots,-x_{S}\right) d_{\underline{b}}^{-1} \chi_{C}(\underline{b})
$$

Inverting

$1 / 2\left[\frac{\partial^{2}}{\partial^{2} y}\left(\frac{1}{x(x+y)}\right)+\left(\frac{\partial}{\partial x}+\frac{\partial}{\partial y}\right)^{2}\left(\frac{1}{(x+y)(-x+y)}\right)-\frac{\partial^{2}}{\partial^{2} x}\left(\frac{1}{y(x+y)}\right)\right]$

we get

$$
1 / 2\left[y^{2} \chi_{C((1,0),(1,1))}+\frac{(x+y)^{2}}{2} \chi_{C((1,1),(-1,1)}-x^{2} \chi_{C((0,1),(1,1))}\right] .
$$

The same analysis for the ZP element leads to Fig. 1.

\subsection{The theory of Dahmen-Micchelli}

Given a vector $a$ denote by $D_{a}$ the directional derivative, a first order operator. For a list $Y$ of vectors set $D_{Y}:=\prod_{a \in Y} D_{a}$, a differential operator of order $|Y|$ with constant coefficients.

We now fix, as usual, a list $X$ of vectors spanning $V$.

For a given unbroken basis $\underline{b}$, consider the element $D_{\underline{b}}:=\prod_{a \notin \underline{b}} D_{a}$. The polynomials $p_{\underline{b}, X}$ are characterized by the differential equations

$$
D_{Y} p=0, \forall Y, \quad \text { a cocircuit in } X, \quad D_{\underline{b}} p_{\underline{c}, X}\left(x_{1}, \ldots, x_{S}\right)=\left\{\begin{array}{lll}
1 & \text { if } \quad \underline{b}=\underline{c} \\
0 & \text { if } \underline{b} \neq \underline{c}
\end{array}\right.
$$


5.4.1. A remarkable space of polynomials.

$$
D(X):=\left\{p \mid D_{Y} p=0, \forall Y, \quad \text { a cocircuit in } X\right\}
$$

By a theorem of Dhamen Micchelli $\operatorname{dim} D(X)$ equals the total number of bases extracted from $X$, the polynomials $p_{\underline{b}}, X$ form a basis for the part of top degree $m-n$ of $D(X)$.

The graded dimension of $D(X)$ is given by $H_{X}(q)=\sum_{b \in \mathcal{B}(X)} q^{m-n(\underline{b})}$. The number $n(\underline{b})$ (called external activity in the Theory of Matroids) is the number of elements of $X$ breaking $\underline{b}$.

For $A_{3}$ the graded dimension is: $6 q^{3}+6 q^{2}+3 q+1$. Remark that for all polynomials in three variables the generating function for the dimension is: . . $+10 q^{3}+6 q^{2}+3 q+1$.

\section{Approximation}

The aim of this section is to explain some motivations, for the study of box-splines, coming from numerical analysis.

\subsection{The Strang-Fix conditions}

The interest of the space of polynomials $D(X)$ comes in approximation theory from the problem of studying the approximation of a function $f(x)$, on $\mathbb{R}^{s}$, by the finite element method (cf. Strang and Fix [33]). Using a box-spline we construct the approximations:

$$
B_{X} *^{\prime} f:=f(x) \mapsto \sum_{\underline{i} \in \mathbb{Z}^{s}} B_{X}(x-\underline{i}) f(\underline{i}), \text { semi-discrete convolution. }
$$

Or at order $n \in \mathbb{N}$ :

$$
f(x) \mapsto \sum_{\underline{i} \in \mathbb{Z}^{s}} B_{X}(n x-\underline{i} / n) f(\underline{i} / n)
$$

In general, given a function $M(x)$ with compact support (as $B_{X}$ ) we want to find optimal weights $c_{\underline{\underline{i}}}(n)$ in order to approximate $f$ by $f(x) \mapsto \sum_{\underline{i} \in \mathbb{Z}^{s}} M(n x-\underline{i} / n) c_{\underline{i}}(n)$ and determine a constant $k \in \mathbb{N}$ so that on any given bounded region there is a constant $C$, independent of $n$ with:

$$
\left|f(x)-\sum_{\underline{i} \in \mathbb{Z}^{s}} M(n x-\underline{i} / n) c_{\underline{i}}(n)\right| \leq C n^{-k}, \quad \forall n .
$$

The maximum $k$ is the approximation power of $M(x)$. 
Given a spline $M(x)$ on $\mathbb{R}^{s}$, with compact support one may define the cardinal spline space to be the space $\mathcal{S}_{M}$ of all (infinite) linear combinations:

$$
\mathcal{S}_{M}:=\left\{\sum_{\underline{i} \in \mathbb{Z}^{s}} M(x-\underline{i}) c_{\underline{i}}\right\} .
$$

The approximation power of $M(x)$ is related to two questions:

1. For which polynomials $f(x)$ we have that $\sum_{i \in \mathbb{Z}^{s}} M(x-\underline{i}) f(\underline{i})$ is a polynomial?

2. Which polynomials lie in the cardinal spline space?

Theorem 8 A polynomial $f(x)$ is in the cardinal spline space of $B_{X}$ if and only if

$$
D_{Y} f=0, \forall Y \subset X \mid \text { the span of } X \backslash Y \text { is not } V
$$

In other words $D_{Y} f=0, \forall Y \subset X$ which are cocircuits.

The Strang-Fix conditions is a general statement:

The approximation power of a function $M$ is the maximum $r$ such that the space of all polynomials of degree $\leq r$ is contained in the cardinal space $\mathcal{S}_{M}$.

For the cardinal spline space in the case $B_{X}(x)$ with $X$ integral we have:

1. $D(X)$ is characterized as the space of polynomials $f(x)$ which reproduce, i.e. map to polynomials under the semi-discrete convolution.

2. $D(X)$ is also characterized as the space of polynomials lying in the cardinal spline space.

The power of approximation by discrete convolution is measured by the maximum degree of the space of polynomials which reproduce under discrete convolution.

6.1.1. Superfunctions. Consider the following algorithm applied to a function $g$ :

$$
g_{h}:=\sum_{\underline{i} \in \Lambda} F(x / h-\underline{i}) g(h \underline{i}), \quad h=1,2, \ldots
$$

There are functions $F$ in the cardinal spline space such that this transformation is the identity on polynomials of degree $<m(X)$, these are the super-functions. For such functions the previous algorithm satisfies the requirements of the Strang-Fix approximation

Theorem 9 We have, under the explicit algorithm previously constructed that, for any domain $G$ :

$$
\left\|f_{h}-f\right\|_{L^{\infty}(G)}=O\left(h^{m(X)}\right) .
$$

For every multi-index $\alpha \in \mathbb{N}^{s}$ with $|\alpha| \leq m(X)-1$, we have:

$$
\left\|\partial^{\alpha} f_{h}-\partial^{\alpha} f\right\|_{L^{\infty}(G)}=\left\|\partial^{\alpha}\left(f_{h}-f\right)\right\|_{L^{\infty}(G)}=O\left(h^{m(X)-|\alpha|}\right) .
$$


Theorem 10 When $p \in D(X)$ we have that also $B_{X} *^{\prime} p \in D(X)$.

This defines a linear isomorphism $F$ of $D(X)$ to itself, given explicitly by the invertible differential operator $\prod_{a \in X} \frac{1-e^{-D_{a}}}{D_{a}}$.

- If $R$ is any difference operator inverting $B_{X} *^{\prime}-$ on $D(X)$ then $R B_{X}$ is a superfunction.

- We can then take as $R$ a truncation of

$$
Q:=\prod_{a \in X}\left(\sum_{i=0}^{\infty} \frac{\nabla_{a}^{i}}{i+1}\right)
$$

Let us give as example some cases of the function $s_{m}(x)$ obtained by this procedure from $b_{m}(x)$.
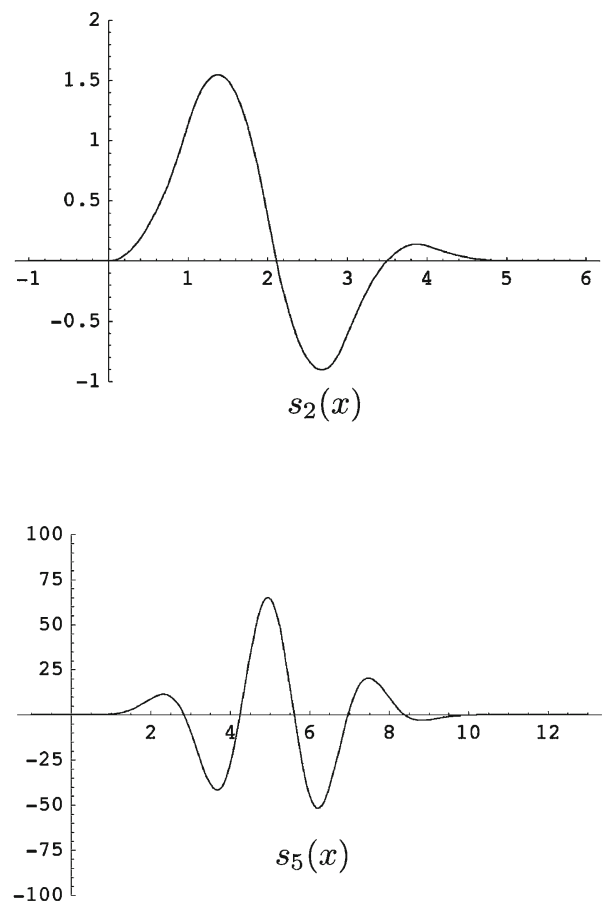


\section{Part 2. Arithmetic and combinatorics}

\section{Arithmetic}

\subsection{The partition function}

The partition function is a quasi polynomial on each big cell, in fact on the larger neighborhood $\mathfrak{c}-B(X)$ of the big cell $\mathfrak{c}$.

We think of the partition function $P_{X}(b)=\#\left\{t_{1}, \ldots, t_{m} \in \mathbb{N} \mid \sum_{i=1}^{m} t_{i} a_{i}=b\right\}$ as a distribution $\sum_{\lambda \in \Lambda} P_{X}(\lambda) \delta_{\lambda}$ with Laplace transform $\sum_{\lambda \in \Lambda} P_{X}(\lambda) e^{-\lambda}=$ $\prod_{a \in X} \frac{1}{\left(1-e^{-a}\right)}$.

There are explicit formulas relating the partition function $P_{X}$ with the multivariate splines $T_{A}, A \subset X$. One could derive the Formula of Example 2.7 in this way.

We used hyperplane arrangements in order to study multivariate splines, for partition functions we use toric arrangements.

\subsection{The toric arrangement}

Let $T$ be the torus of character group $\Lambda, X$ a list of characters $u_{X}=\prod_{a \in X}\left(1-e^{a}\right)$ and $S_{X}=\mathbb{C}[\Lambda]\left[u_{X}^{-1}\right]$ is the coordinate ring of the open set $P_{X} \subset T$ complement of the union of the subgroups of $T$ of equations $e^{a}=1, a \in X$. If $a \in \mathbb{Z}^{s}$ is a vector of coordinates $\left(n_{1}, \ldots, n_{s}\right)$ we also write $e^{a}=\prod_{i=1}^{s} x_{i}^{n_{i}}$.

The toric arrangement is the finite set consisting of all the connected components of the subvarieties obtained by intersecting the subgroups of $T$ of equations $e^{a}=1, a \in X$.

EXAMPLE $s=1, T=\mathbb{C}^{*}, X=\{5,3\}$

The arrangement consists of the connected components of the variety $x^{5}=1$ or $x^{3}=1$, i.e. of the five, fifth roots of 1 and the three third roots of 1 .

The elements of the toric arrangement are ordered by reverse inclusion, particular importance is given to the points of the arrangement, $P(X)$ which are the zerodimensional, i.e. points, elements of the arrangement.

A very special case is when $P(X)$ reduces to the point 1 , this is the unimodular case. For root systems the only unimodular case is $A_{n}$.

Each point $p \in P(X)$ determines a sublist:

$$
X_{p}:=\left\{a \in X \mid e^{a}(p)=1\right\} .
$$

\section{EXAMPLE ZP}

$$
X=\left|\begin{array}{cccc}
1 & 1 & 0 & -1 \\
1 & 0 & 1 & 1
\end{array}\right|
$$

The subgroups are

$$
x y=1, \quad x=1, \quad y=1, \quad x^{-1} y=1 .
$$


We have two points in $P(X)$

$$
\begin{gathered}
(1,1), \quad(-1,-1) . \\
X_{(1,1)}=X, \quad X_{(-1,-1)}=\left|\begin{array}{cc}
1 & -1 \\
1 & 1
\end{array}\right| .
\end{gathered}
$$

\subsection{The filtration}

The formulas we are aiming at are built from basic functions. These are analogues, in the discrete case, of the Laplace transforms of the characteristic functions of the cones generated by bases extracted from $X$. Given a basis $\underline{b}$ of $V=\Lambda \otimes \mathbb{R}$, contained in $\Lambda$, we introduce the set

$$
R_{\underline{b}}=\left\{\lambda \in \Lambda \mid \lambda=\sum_{b \in \underline{b}} p_{b} b, \text { with } 0 \leq p_{b}<1\right\} .
$$

Notice that this is a set of coset representatives of $\Lambda \cap\langle\underline{b}\rangle / \Lambda_{\underline{b}}$. Given a character $\phi: \Lambda \rightarrow \mathbb{C}^{*}$ which is 1 on $\underline{b}$ set

$$
\xi_{\phi}:=\sum_{v \in \Lambda \cap C(\underline{b})} e^{\langle\phi \mid v\rangle} \delta_{v}
$$

$\xi_{\phi}$ is a tempered distribution supported on the cone $C(\underline{b})$ and its Laplace transform has an analytic meaning and we get:

Proposition 7.4 The Laplace transform of $\xi_{\phi}$ equals

$$
\begin{gathered}
|\operatorname{det}(\underline{b})| \frac{e(\phi)}{\prod_{a \in \underline{b}}\left(1-e^{-a}\right)} . \\
e(\phi):=\frac{\sum_{\lambda \in R_{\underline{b}}} e^{\langle\phi \mid \lambda\rangle} e^{-\lambda}}{|\operatorname{det}(\underline{b})|}
\end{gathered}
$$

We filter $S_{X}$ by polar order that is $S_{X, k}$ is formed by all fractions which have at the denominator a product $\prod_{a \in X}\left(1-e^{a}\right)^{h_{a}}$ so that the $a$ with $h_{a}>0$ span a subspace of dimension $\leq k$.

A connected component of the toric arrangement is of the form $p P$ for some subtorus $P$. For each connected component $p P$ we have an irreducible module generated by the delta function. 
Concretely, if $p P$ has codimension $k$ we construct a copy $N_{p P, \underline{b}}$ in $S_{X, k} / S_{X, k-1}$ as generated by the class of an element $|\operatorname{det}(\underline{b})| \frac{e(\phi)}{\prod_{a \in \underline{b}}\left(1-e^{-a}\right)}$ as $\underline{b}$ is a basis for the span of the sublist $X_{p P}$ of the elements in $X$ which are 1 on $p P$.

Theorem $11 S_{X, k} / S_{X, k-1}$, decomposes as direct sum of the modules $N_{p P, \underline{b}}$ as $p P$ runs over all components of the arrangement of codimension $k$ and $\underline{b}$ over the unbroken bases on $p P$.

For each $p P, F_{p P}$ is the isotypic component of type $N_{p P}$.

Take the space of polar parts $\mathcal{S} P_{X}:=S_{X} / S_{X, s-1}$, its isotypic components are $F_{\phi}$ indexed by points of the arrangement.

$$
\mathcal{S} P_{X}:=S_{X} / S_{X, s-1}=\bigoplus_{\phi \in \tilde{P}(X)} F_{\phi}, \quad F_{\phi}=\bigoplus_{\underline{b} \in \mathcal{N} \mathcal{B}_{X_{\phi}} N_{b}, e^{\phi}} .
$$

Let us consider the element $v_{X}$, in $\mathcal{S} P_{X}$, class of the function $\prod_{a \in X}\left(1-e^{-a}\right)^{-1}$. Decompose it uniquely as a sum of elements $v_{X_{\phi}}$ in $F_{\phi}$, each one of these elements is uniquely expressed as a sum

$$
v_{X_{\phi}}=\sum_{\underline{b} \in \mathcal{N} \mathcal{B}_{X_{\phi}}} \mathfrak{q}_{\underline{b}, \phi} \omega_{\underline{b}, \phi}
$$

for suitable polynomials $\mathfrak{q}_{\underline{b}, \phi} \in S\left[U_{\mathbb{C}}\right]$. Thus:

$$
v_{X}=\sum_{\phi \in \tilde{P}(X)} \sum_{\underline{b} \in \mathcal{N} \mathcal{B}_{X_{\phi}}} \mathfrak{q}_{\underline{b}, \phi} \omega_{\underline{b}, \phi}
$$

The main formula that one can effectively use for computing the partition function $P_{X}$ :

Theorem 12 Let $\Omega$ be a big cell, $B(X)$ the box associated to $X$. For every $x \in$ $\Omega-B(X)$,

$$
P_{X}(x)=\sum_{\phi \in \tilde{P}(X)} e^{\langle\phi \mid x\rangle} \sum_{\underline{b} \in \mathcal{N} \mathcal{B}_{X_{\phi}} \mid \Omega \subset C(\underline{b})}|\operatorname{det}(\underline{b})|^{-1} \mathfrak{q}_{\underline{b}, \phi}(-x) .
$$

Finally we compute the contributions by residues.

The isotypic components appearing in grade $k$ correspond to the connected components of the toric arrangement of codimension $k$.

For the top part $S_{X, n} / S_{X, n-1}$ d we have a sum over the points of the arrangement $P(X)$.

The isotypic component associated to a point $e^{\phi}$ decomposes as direct sum of irreducibles indexed by the unbroken bases in $X_{\phi}:=\left\{a \in X \mid e^{\langle a \mid \phi\rangle}=1\right\}$. 
7.4.1. Local structure of $P_{X}$. The previous formula shows in particular, that the partition function is on each cell a quasi polynomial, as for the case of the multivariate spline:

The quasi polynomials appearing in the formula for $P_{X}$ satisfy special difference equations.

Denote by $\mathcal{C}[\Lambda]$ the abelian group of $\mathbb{Z}$-valued functions on $\Lambda$.

If $a \in \Lambda$, the difference operator $\nabla_{a}(f)(x):=f(x)-f(x-a)$ acts on $\mathcal{C}[\Lambda]$. Given $X$ a list in $\Lambda$ spanning the ambient space we define

\section{Definition 7.5}

$$
\operatorname{DM}(X):=\left\{f \in \mathcal{C}[\Lambda] \mid \nabla_{Y} f=\prod_{a \in Y} \nabla_{a} f=0\right\}
$$

as $Y$ runs over all cocircuits of $X$.

7.5.1. Second Theorem of Dahmen-Micchelli. The dimension of $D M(X)$ is the Volume $\delta(X)$ of the box $B(X)$ :

$$
\delta(X)=\sum_{\underline{b} \in \mathcal{B}(X)}|\operatorname{det}(\underline{b})| .
$$

This follows from a more precise statement.

Theorem 13 If $r$ is a regular vector $\delta(r \mid X):=r-B(X) \cap \Lambda$ has cardinality $\delta(X)$. The restriction of $D M(X)$ to $\mathbb{Z}$ valued functions on $\delta(r \mid X)$ is an isomorphism.

In other words the values on $\delta(r \mid X)$ of a function in $D M(X)$ determine uniquely this function and in particular $D M(X)$ is a free abelian group of rank $\delta(X)$.

Example 7.6 (The binomial coefficients) $X=\underbrace{\{1,1, \ldots, 1\}}_{m+1}$ the zonotope $B(X)=$ $[0, m+1]$ is the interval.

In this case we only have one equation

$$
\nabla_{1} f(x)=f(x)-f(x-1), \quad \nabla_{1}^{m+1} f=0 .
$$

The space of solutions has as integral basis the elements

$$
\left(\begin{array}{c}
x+k \\
m
\end{array}\right)=\frac{(x+k)(x+k-1) \ldots(x+k-m+1)}{m !}, \quad k=0, \ldots, m .
$$

Take a regular point close to $0,0<a<1$ then

$$
\begin{gathered}
\delta(a \mid X)=a-[0, m+1] \cap \mathbb{Z}=[-m-1+a, a] \cap \mathbb{Z}=[-m, \ldots, 0] . \\
\left(\begin{array}{c}
i+k \\
m
\end{array}\right)=0, \quad i \in[-k, \ldots, m-1-k], \quad\left(\begin{array}{c}
m-k+k \\
m
\end{array}\right)=1 .
\end{gathered}
$$


The partition function is $\left(\begin{array}{c}x+m \\ m\end{array}\right)$. The matrix of the values of $\left(\begin{array}{c}x+i \\ m\end{array}\right)$ for $i \in[0, m]$ at the points $0,-1, \ldots,-m$ are given by the matrix, invertible over $\mathbb{Z}$ and establishes the isomorphism of Theorem 13:

$$
m=10,\left(\begin{array}{lllllllllll}
92378 & 43758 & 19448 & 8008 & 3003 & 1001 & 286 & 66 & 11 & 1 & 0 \\
43758 & 19448 & 8008 & 3003 & 1001 & 286 & 66 & 11 & 1 & 0 & 0 \\
19448 & 8008 & 3003 & 1001 & 286 & 66 & 11 & 1 & 0 & 0 & 0 \\
8008 & 3003 & 1001 & 286 & 66 & 11 & 1 & 0 & 0 & 0 & 0 \\
3003 & 1001 & 286 & 66 & 11 & 1 & 0 & 0 & 0 & 0 & 0 \\
1001 & 286 & 66 & 11 & 1 & 0 & 0 & 0 & 0 & 0 & 0 \\
286 & 66 & 11 & 1 & 0 & 0 & 0 & 0 & 0 & 0 & 0 \\
66 & 11 & 1 & 0 & 0 & 0 & 0 & 0 & 0 & 0 & 0 \\
11 & 1 & 0 & 0 & 0 & 0 & 0 & 0 & 0 & 0 & 0 \\
1 & 0 & 0 & 0 & 0 & 0 & 0 & 0 & 0 & 0 & 0 \\
0 & 0 & 0 & 0 & 0 & 0 & 0 & 0 & 0 & 0 & 1
\end{array}\right) .
$$

Formula (11) has a strict connection with the paving of the box.

Example 7.7 Let us take

$$
X=\left|\begin{array}{cccc}
0 & 1 & 1 & -1 \\
1 & 0 & 1 & 1
\end{array}\right|
$$

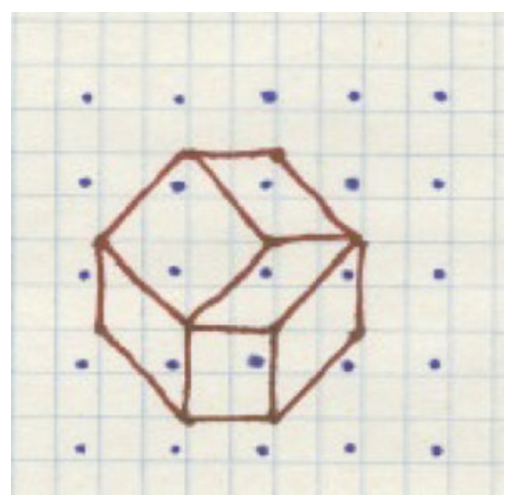

See that $\delta(X)=1+1+1+1+1+2=7$ is the number of points in which the box $B(X)$, shifted generically a little, intersects the lattice!

\subsection{Combinatorics}

$\Lambda$ is a lattice in a real vector space $V$ and $X$ a list of vectors in $\Lambda$. In coordinates $V=\mathbb{R}^{s}, \Lambda=\mathbb{Z}^{s}$. Assume that $X$ generates a pointed cone $C(X)$. We have already seen in Sect. 2.9 the notions of singular, regular points and big cells. We now extend these notions as follows: 
(i) The translates $C^{\text {sing }}(X)+\Lambda$ give a periodic hyperplane arrangement.

(ii) The union of the hyperplanes of this periodic arrangement is called the cut locus and each connected component of its complement a chamber.

(iii) Each chamber of this arrangement is the interior of a bounded polytope.

(iv) The function $B_{X}$ is a polynomial on each chamber.

(v) The set of chambers is invariant under translation by elements of $\Lambda$.

Example $7.9 X=\left|\begin{array}{cccc}1 & 1 & 0 & -1 \\ 1 & 0 & 1 & 1\end{array}\right|$,

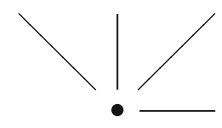

When we translate we have:

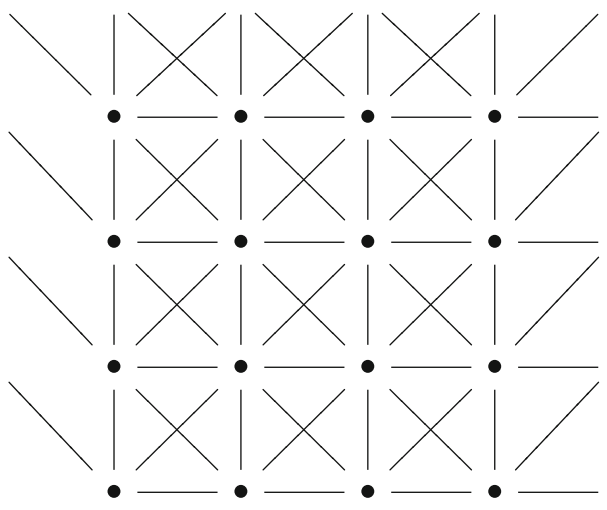

We draw the zonotope and the cut-locus

If $X$ is a basis of the lattice $\Lambda$ we have that the chambers are open parallelepipeds. 


\section{Difference theorem}

One of the main results of the Theory is the fact that a partition function $P_{X}$ is a quasi-polynomial on the regions $\Omega-B(X)$, for each big cell $\Omega$.

The fact that $P_{X}$ is a quasi-polynomial not just on the big cells but in fact in the larger regions $\Omega-B(X)$ may be considered as a discrete analogue of the differentiability properties of the multivariate spline $T_{X}$. In this section we discuss some properties of the quasi-polynomials describing $P_{X}$.

- $P_{X}$ on $\Omega-B(X)$ coincides with an element of $D M(X)$.

- The element is uniquely determined by its initial values, that is the values it takes on $\delta(u \mid X)$ where $u \in \Omega$ is close to zero.

- In this case $\delta(u \mid X) \cap C(X)=\{0\}$ so that, in order to coincide with $P_{X}$ on $\Omega-B(X)$ we must have that $f(0)=1$ while $f(a)=0$ for all non-zero elements of $\delta(u \mid X)$.

- These are the defining initial conditions.

An idea of a possible proof will be given in Sect. 9.20.

Let us start to explain why the system of difference equations gives a system of recursion which allow to determine the values of a function $f \in D M(X)$ by the values that it takes in a set $\delta(u \mid X)$. To begin notice that

$$
\tau_{a} \tau_{b}=\tau_{a+b} \Longrightarrow \prod_{i=1}^{m} \nabla_{a_{i}}=\sum_{S \subset\{1, \ldots, m\}}(-1)^{|S|} \tau_{\left(\sum_{i \in S} a_{i}\right)} .
$$

Thus the recursive formula for a function $f(x)$ satisfying $\prod_{i=1}^{m} \nabla_{a_{i}} f=0$.

$$
f(x)=\sum_{S \subset\{1, \ldots, m\}, S \neq \emptyset}(-1)^{|S|+1} f\left(x-\sum_{i \in S} a_{i}\right) .
$$

Example $8.1 X=\{2,3\}, \nabla_{2}=1-\tau_{2}, \nabla_{3}=1-\tau_{3}$, so

$$
\nabla_{2} \nabla_{3} f(x)=f(x)-f(x-2)-f(x-3)+f(x-5)
$$

easily implies that a function satisfying $\nabla_{2} \nabla_{3} f(x)=0$ is determined by the values that it takes on the interval $[-4,0]$.

The partition function $P_{2,3}$ for the list 2,3 is characterized by the fact that: for every number $i>-5$, the function $P_{2,3}$ coincides with the unique function $f(x)$ which satisfies the difference equation $\nabla_{2} \nabla_{3} f(x)=0$ and which takes values $0,0,0,0,1$ on $-4,-3,-2,-1,0$.

A function $f$ satisfying $\nabla_{Y} f=$ for all cocircuits $Y$ extracted from $X$ is determined by the values on a set $\delta\left(\mathfrak{c}_{0} \mid X\right)$ where $\mathfrak{c}_{0}$ is a given chamber. 
- First $\Lambda=\cup_{\mathfrak{c}} \delta(\mathfrak{c} \mid X)$ as $\mathfrak{c}$ runs over all chambers.

- Next we want to prove that if $f$ vanishes on a given $\delta(\mathfrak{c} \mid X)$ for a chamber $\mathfrak{c}$ then it also vanishes on $\delta(\mathfrak{g} \mid X)$ for a chamber $\mathfrak{g}$ which is adjacent to $\mathfrak{c}$.

- Then we go by recursion.

The fact that the values of a function $f \in D M(X)$ are determined by the values it takes on a single set $\delta(\mathfrak{c} \mid X)$ comes from a wall crossing formula when we pass from $\delta(\mathfrak{c} \mid X)$ to $\delta(\mathfrak{g} \mid X)$ for a chamber $\mathfrak{g}$ which is adjacent to $\mathfrak{c}$.

\subsection{Arithmetic}

8.2.1. Connection between $D M(X)$ and $D(X)$. There is a formal machinery which allows us to interpret, locally around a point, difference equations as restriction to the lattice of differential equations, we call it the logarithm isomorphism. We have this for any module over the periodic Weyl algebra $\mathbb{C}\left[\frac{\partial}{\partial x_{i}}, e^{x_{i}}\right]$ as soon as for algebraic reasons (nilpotency) we can deduce from the action of $e^{x_{i}}$ also an action of $x_{i}$.

The main point is that the ideal $J_{X}$ generated by the elements $\prod_{a \in Y}\left(1-e^{a}\right)$ as $Y$ runs over the cocircuits defines (as a scheme) the set $P(X)$ of points of the arrangement.

Thus

$$
\mathbb{C}[\Lambda] / J_{X}=\oplus_{p \in P(X)} \mathbb{C}[\Lambda] / J_{X}(p) .
$$

Where $\mathbb{C}[\Lambda] / J_{X}(p)$ is a local algebra supported at $p$.

This implies that the dual of $\mathbb{C}[\Lambda] / J_{X}$ which is the complexified form

$$
D M_{\mathbb{C}}(X)=D M(X) \otimes \mathbb{C}
$$

decomposes as direct sum of contributions at the various points in $P(X)$.

Choose representatives $\tilde{P}(X)$ for $P(X)$ :

$$
D M_{\mathbb{C}}(X)=\oplus_{\phi \in \tilde{P}(X)} e^{\langle\phi \mid v\rangle} D_{\phi}
$$

where $D_{\phi}$ is the space of polynomials $f$ such that $e^{\langle\phi \mid v\rangle} f \in D M_{\mathbb{C}}(X)$.

Proposition 8.3 The space $D_{\phi}$ is the differentiable Dahmen-Micchelli space $D\left(X_{p}\right)$, $p=e^{\phi}$.

This explains why $D M(X)$ is formed by quasi-polynomials.

- if $v \in V$, the difference operator $\nabla_{v}$ and the differential operator $\partial_{v}$ satisfy $\nabla_{v}=$ $T_{v} \partial_{v}$ with $T_{v}=\frac{1-e^{-\partial_{v}}}{\partial_{v}}$.

- $T_{v}$ is invertible on the space of polynomials and commutes with all the $\nabla_{a}$.

- Hence $\nabla_{Y}=A D_{Y}$ where $A$ is invertible on polynomials. 
- The equations $\nabla_{Y} f=0$ are equivalent to $D_{Y} f=0$ for polynomials.

- Thus we obtain that $e^{\langle\phi \mid v\rangle} f \in D M_{\mathbb{C}}(X)$ if and only if $f \in D\left(X_{p}\right)$.

$$
D M_{\mathbb{C}}(X)=\oplus_{\phi \in \tilde{P}(X)} e^{\langle\phi \mid v\rangle} D\left(X_{e^{\phi}}\right)
$$

- In particular the contribution of the point 1 to $D M_{\mathbb{C}}(X)$ is the space of polynomials $D(X)$.

- In the unimodular case $D M_{\mathbb{C}}(X)=D(X)$.

- In general we also have the contribution of the other points, that will be denoted by

$$
E(X):=\oplus_{p \in P(X), p \neq 1} e^{\langle\phi \mid v\rangle} D\left(X_{p}\right) .
$$

8.3.1. Supports. We want now to explain the nature of $D M_{\mathbb{C}}(X)$ as Fourier coefficients of distributions on the compact torus $T$, supported at the points of the arrangement.

For this we consider the space $D(X)$ (or $D\left(X_{p}\right)$ ) as a space of polynomial differential operators on $U$ and hence also on $T$ with constant coefficients.

The Haar measure on $T$ of total mass 1 . This allows us to identify generalized functions on $T$ and distributions on $T$.

Call $\widehat{D M}(X)$ the space of distributions on $T$ of which $D M_{\mathbb{C}}(X)$ gives the Fourier coefficients.

We deduce

Proposition 8.4 $\widehat{D M}(X)$ is the direct sum of the spaces of distributions $D\left(X_{p}\right) \delta_{p}$ as $p \in P(X)$.

In particular we see that $\widehat{D M}(X)$ is supported at the finite set $P(X)$.

\section{Residues}

\subsection{Wonderful models}

There is an approach to compute the partition function based on residues (as for numbers in Sect. 2.4.1), also in higher dimension.

In order to define multidimensional residues we need divisors with normal crossings. That is we consider functions which, in some coordinates $x_{i}$, have poles only on the hyperplanes $x_{i}=0, i=1, \ldots, s$. The residue is the coefficient of $\prod_{i} x_{i}^{-1}$.

The construction of these divisors with normal crossings follows [16] and in our case starts with a

Definition 9.2 Given a subset $A \subset X:=\left\{x_{1}, \ldots, x_{s}\right\}$ the list $\bar{A}:=X \cap\langle A\rangle$ will be called the completion of $A$. In particular $A$ is called complete if $A=\bar{A}$.

The space of vectors $\phi \in U$ such that $\langle a \mid \phi\rangle=0$ for every $a \in A$ will be denoted by $A^{\perp}$. Notice that clearly $\bar{A}$ equals to the list of vectors $a \in X$ which vanish on $A^{\perp}$. 
From this we see that we get a bijection between the complete subsets of $X$ and subspaces of the arrangement defined by $X$.

A central notion in what follows is given by

Definition 9.3 Given a complete set $A \subset X$, a decomposition is a decomposition $A=A_{1} \cup A_{2}$ in non empty sets, such that:

$$
\langle A\rangle=\left\langle A_{1}\right\rangle \oplus\left\langle A_{2}\right\rangle .
$$

Clearly the two sets $A_{1}, A_{2}$ are necessarily complete.

We shall say that: a complete set $A$ is irreducible if it does not have a non trivial decomposition.

Theorem 14 Every set $A$ can be decomposed as $A=A_{1} \cup A_{2} \cup \cdots \cup A_{k}$ with the $A_{i}$ irreducible and:

$$
\langle A\rangle=\left\langle A_{1}\right\rangle \oplus\left\langle A_{2}\right\rangle \oplus \cdots \oplus\left\langle A_{k}\right\rangle
$$

This decomposition is unique up to order.

$A=A_{1} \cup A_{2} \cup \cdots \cup A_{k}$ is called the decomposition into irreducibles of $A$.

Example 9.4 An interesting example is that of the configuration space of $s$-ples of point in a line (or the root system $A_{s-1}$ ). In this case $X=\left\{z_{i}-z_{j} \mid 1 \leq i<j \leq s\right\}$.

In this case, irreducible sets are in bijection with subsets of $\{1, \ldots, s\}$ with least 2 elements. If $S$ is such a subset the corresponding irreducible is $I_{S}=\left\{z_{j}-z_{j} \mid\{i, j\} \subset S\right\}$.

Given a complete set $C$, the irreducible decomposition of $C$ corresponds to a family of disjoint subsets $S_{1}, \ldots, S_{k}$ of $\{1, \ldots, s\}$ each with at least 2 elements.

Definition 9.5 A family $\mathcal{S}$ of irreducibles $A_{i}$ is called nested if, given elements $A_{i_{1}}, \ldots, A_{i_{h}} \in \mathcal{S}$ mutually incomparable we have that $C:=A_{1} \cup A_{2} \cup \cdots \cup A_{i}$ is complete and $C:=A_{1} \cup A_{2} \cup \cdots \cup A_{i}$ is its decomposition into irreducibles.

Consider the hyperplane arrangement $\mathcal{H}_{X}$ and the open set

$$
\mathcal{A}_{X}=U /\left(\cup_{H \in \mathcal{H}_{X}} H\right)
$$

complement of the union of the given hyperplanes.

Let us denote by $\mathcal{I}$ the family of irreducible subsets in $X$.

We construct a minimal smooth variety $Z_{X}$ containing $\mathcal{A}_{X}$ as an open set with complement a normal crossings divisor, plus a proper map $\pi: Z_{X} \rightarrow U$ extending the identity of $\mathcal{A}_{X}$.

For any irreducible subset $A \in \mathcal{I}$ take the vector space $V / A^{\perp}$ and the projective space $\mathbb{P}\left(V / A^{\perp}\right)$.

Notice that, since $A^{\perp} \cap \mathcal{A}_{X}=\varnothing$ we have a natural projection $\pi_{A}: \mathcal{A}_{X} \rightarrow$ $\mathbb{P}\left(V / A^{\perp}\right)$. If we denote by $j: \mathcal{A}_{X} \rightarrow U$ the inclusion we get a map The model

$$
i:=j \times\left(\times_{a \in \mathcal{I}} \pi_{A}\right): \mathcal{A}_{X} \rightarrow U \times\left(\times_{a \in \mathcal{I}} \mathbb{P}\left(U / A^{\perp}\right)\right)
$$


Definition 9.6 The model $Z_{X}$ is the closure of $i\left(\mathcal{A}_{X}\right)$ in $U \times\left(\times_{a \in \mathcal{I}} \mathbb{P}\left(U / A^{\perp}\right)\right)$.

There is a very efficient approach to computations by residue at points at infinity in the wonderful compactification of the associated hyperplane arrangement.

Points at infinity correspond to maximal nested sets. Around each such point one can consider a $s$-dimensional torus and its class in homology

A basis of the homology or of the corresponding residues corresponds to the tori around special points indexed by unbroken bases.

9.6.1. The non linear coordinates. We now apply the previous Theory to the multivariate spline or the partition function associated to a list $X$. One can find explicit polynomials $p_{b, X}(x)$ (given in Formula (16)), indexed by the points at infinity associated to the maximal nested set generated by unbroken bases so that, given a point $x$ in the closure of a big cell $\mathfrak{c}$ we have Jeffrey-Kirwan residue formula [26].

$$
T_{X}(x)=\sum_{\underline{b} \mid \mathfrak{c} \subset C(\underline{b})}|\operatorname{det}(\underline{b})|^{-1} p_{\underline{b}, X}(-x) .
$$

There is a parallel theory for the partition function, as a result we can compute a set of polynomials $q_{b, \phi}(-x)$ (given in Formula (17)) indexed by pairs, a character $\phi$ of finite order and a unbroken basis in $X_{\phi}=\left\{a \in X \mid \phi\left(e^{a}\right)=1\right\}$.

The analogue of the Jeffrey-Kirwan formula is:

Theorem 15 Given a point $x$ in the closure of a big cell $\mathfrak{c}$ we have a Residue formula for partition function

$$
P_{X}(x)=\sum_{\phi \in P(X)} e^{\phi} \sum_{\underline{b} \in \mathcal{N} \mathcal{B}_{X_{\phi}} \mid \mathfrak{c} \subset C(\underline{b})} \mathfrak{q}_{\underline{b}, \phi}(-x)
$$

Finally one can deduce the partition function from some combinatorics and multivariate splines (with parameters):

Theorem 16 For the points $x$ in the interior of big cells $\mathfrak{c}$ we have

$$
\begin{gathered}
P_{X}(x)=\sum_{\phi \in P(X)} \widehat{Q}_{\phi} T_{X_{\phi}, \underline{\phi}}(x) \\
Q_{\phi}=\prod_{a \notin X_{\phi}} \frac{1}{1-e^{-a}} \prod_{a \in X_{\phi}} \frac{a-\langle\phi \mid a\rangle}{1-e^{-a+\langle\phi \mid a\rangle}}
\end{gathered}
$$

9.6.2. Residues. The polynomials building the multivariate spline and the partition functions are residues.

Given a MNS $\mathcal{S}$ choose a basis $\underline{b}:=b_{1}, \ldots, b_{s}$ from $X$ so that if $X_{i}$ is the minimal element of $\mathcal{S}$ containing $b_{i}$ we have all the $X_{i}$ distinct. 
Construct new coordinates $z_{A}, A \in \mathcal{S}$ using the monomial expressions:

$$
b_{A}:=\prod_{B \in \mathcal{S}, A \subseteq B} z_{B} .
$$

The residue at the point 0 for these coordinates is denoted by $r e s_{\underline{b}}$.

$$
\begin{aligned}
& p_{\underline{b}, X}(-y)=\operatorname{det}(\underline{b}) \operatorname{res}_{\underline{b}}\left(\frac{e^{\langle y \mid x\rangle}}{\prod_{a \in X}\langle x \mid a\rangle}\right) \text { Spline. } \\
& \mathfrak{q}_{\underline{b}, X_{\phi}}(-y)=\operatorname{det}(\underline{b}) \operatorname{res}_{\underline{b}, \phi}\left(\frac{e^{\langle y \mid z\rangle}}{\prod_{a \in X}\left(1-e^{-a(z)-\langle\phi \mid a\rangle}\right)}\right) \text { Partition function. }
\end{aligned}
$$

Theorem 17 (Brion-Vergne [10]) For every $h=\sum_{\underline{b} \in \mathcal{N B}(X)} q_{\underline{b}, X} d_{\underline{b}}^{-1}$ with $q_{\underline{b}, X} \in S[U]$. We have:

$$
q_{\underline{b}, X}(-y)=\operatorname{det}(\underline{b}) \operatorname{res}_{\underline{b}}\left(e^{\langle y \mid x\rangle} h(x)\right) .
$$

The proof is easy applying formal properties of the residue.

This gives a simple algorithm to compute the polynomials $q_{\underline{b}}, X$.

(i) First make the non-linear change of coordinates $b_{i}=\prod_{j=1}^{i} z_{i}$ in the function $h$.

(ii) You get a Laurent series in the variables $z_{i}$ with coefficients polynomials in the variables $y$.

(iii) The polynomial $q_{\underline{b}, X}(-y)$ appears now as the coefficient of $\prod_{i=1}^{s} z_{i}^{-1}$.

9.7 The abelian group $\mathcal{F}(X)$.

We denote by $\mathcal{C}[\Lambda, \underline{r}]$ the subgroup in $\mathcal{C}[\Lambda]$ consisting of the elements supported in $\Lambda \cap \underline{r}$. The following abelian group will play a key role in what follows

\section{Definition 9.8}

$$
\mathcal{F}(X):=\left\{f \in \mathcal{C}[\Lambda] \mid \nabla_{X \backslash \underline{r}} f \in \mathcal{C}[\Lambda, \underline{r}], \text { for all } \underline{r} \in S_{X}\right\}
$$

Notice that if $f \in \mathcal{F}(X)$ then $f$ must in particular satisfy the relation corresponding to the space $\underline{r}=\{0\})$ that is

$$
\nabla_{X} f=c \delta_{0}
$$

or the equivalent relation

$$
\prod_{a \in X}\left(1-e^{a}\right) L f=c
$$


with $c \in \mathbb{Z}$. In particular $\mathcal{F}(X) \subset \mathcal{R}$ (the rational elements) and $L_{r}(\mathcal{F}(X)) \subset C(\Lambda)$ is the one dimensional abelian group spanned by

$$
\prod_{a \in X} \frac{1}{1-e^{-a}} .
$$

Example 9.9 Let us give a simple example. Let $\Lambda=\mathbb{Z}$, and $X=[2,-1]$. Then it is easy to see that $\mathcal{F}(X)$ has as integral basis

$$
\begin{gathered}
\theta_{1}=\sum_{n \in \mathbb{Z}} \delta_{n}, \quad \theta_{2}=\sum_{n \in \mathbb{Z}} n \delta_{n}, \\
\theta_{3}=\sum_{n \in \mathbb{Z}}\left(\frac{n}{2}+\frac{1-(-1)^{n}}{4}\right) \delta_{n}, \quad \theta_{4}=\sum_{n \geq 0}\left(\frac{n}{2}+\frac{1-(-1)^{n}}{4}\right) \delta_{n} .
\end{gathered}
$$

Here $\theta_{1}, \theta_{2}, \theta_{3}$ is an integral basis of $D M(X)$.

Proposition $9.10 \mathcal{F}(X)$ is a free abelian group whose rank equals the number of integral points in the Zonotope $B(X)$.

Recall that, in Lemma 1.2 we have defined the partition functions $P_{X}^{F}$ associated to the faces of the hyperplane arrangement given by $X$.

The first important fact on this abelian group is the following:

(i) If $F$ is a regular face for $X$, then $P_{X}^{F}$ lies in $\mathcal{F}(X)$.

(ii) The abelian group $D M(X)$ is contained in $\mathcal{F}(X)$.

Proof (i) Indeed, $\nabla_{X \backslash \underline{r}} P_{X}^{F}=P_{X \cap \underline{r}}^{F} \in \mathcal{C}[\Lambda, \underline{r}]$.

(ii) is clear from the definitions.

Each $P_{X}^{F}$ is a partition function. In particular, if $X$ generates a pointed cone, then the partition function $P_{X}$ equals $P_{X}^{F}$ for the face $F$ which is positive on $X$.

9.10.1. Some properties of $\mathcal{F}(X)$. Let $\underline{r}$ be a rational subspace and $F_{\underline{r}}$ be a regular face for $X \backslash \underline{r}$.

Proposition 9.11 (i) The map $g \mapsto P_{X \backslash \underline{r}}^{F_{\underline{r}}} * g$ gives an injection from $\mathcal{F}(X \cap \underline{r})$ to $\mathcal{F}(X)$. Moreover

$$
\nabla_{X \backslash \underline{r}}\left(P_{X \backslash \underline{r}}^{F_{\underline{r}}} * g\right)=g, \forall g \in \mathcal{F}(X \cap \underline{r}) .
$$

(ii) $\nabla_{X \backslash \underline{r}}$ maps $\mathcal{F}(X)$ surjectively to $\mathcal{F}(X \cap \underline{r})$.

(iii) If $g \in D M(X \cap \underline{r})$, then $\nabla_{X \backslash \underline{t}}\left(P_{X \backslash \underline{r}}^{F_{\underline{r}}} * g\right)=0$ for any rational subspace $\underline{t}$ such that $\underline{t} \cap \underline{r} \neq \underline{r}$. 
Recall that $S_{X}^{(i)}$ the set of rational subspaces of dimension $i$. Define the abelian groups

$$
\mathcal{F}(X)_{i}:=\cap_{\underline{t} \in S_{X}^{(i-1)}} \operatorname{ker}\left(\nabla_{X \backslash \underline{t}}\right) \cap \mathcal{F}(X) .
$$

Notice that by definition $\mathcal{F}(X)_{\{0\}}=\mathcal{F}(X)$, that $\mathcal{F}(X)_{\operatorname{dim} V}$ is the abelian group $D M(X)$ and that $\mathcal{F}(X)_{i+1} \subseteq \mathcal{F}(X)_{i}$.

Choose, for every rational space $\underline{r}$, a regular face $F_{\underline{r}}$ for $X \backslash \underline{r}$.

Let $\underline{r} \in S_{X}^{(i)}$.

(i) The image of $\nabla_{X \backslash \underline{r}}$ restricted to $\mathcal{F}(X)_{i}$ is contained in the abelian group $D M(X \cap \underline{r})$.

(ii) If $f$ is in $D M(X \cap \underline{r})$, then $P_{X \backslash \underline{r}}^{F_{\underline{r}}} * f \in \mathcal{F}(X)_{i}$.

Theorem 18 With the previous choices, we have:

$$
\mathcal{F}(X)=\oplus_{\underline{r} \in S_{X}} P_{X \backslash \underline{r}}^{F_{\underline{r}}} * D M(X \cap \underline{r}) .
$$

Definition 9.12 A collection $\mathbf{F}=\left\{F_{\underline{r}}\right\}$ of faces $F_{\underline{r}} \subset \underline{r}^{\perp}$ regular for $X \backslash \underline{r}$, indexed by the rational subspaces $\underline{r} \in S_{X}$ will be called a $X$-regular collection.

Given a $X$-regular collection $\mathbf{F}$, we can write, using Theorem 18, an element $f \in \mathcal{F}(X)$ as

$$
f=\sum_{\underline{r} \in S_{X}} f_{\underline{r}} \text { with } \quad f_{\underline{r}} \in P_{X \backslash \underline{r}}^{F_{\underline{r}}} * D M(X \cap \underline{r}) .
$$

This expression for $f$ will be called the $\mathbf{F}$ decomposition of $f$. In this decomposition, we always have $F_{V}=\{0\}, P_{X \backslash V}^{F_{V}}=\delta_{0}$ and the component $f_{V}$ is in $D M(X)$.

\subsection{Localization theorem}

There is a more restricted notion than that of big cell, it is the notion of tope by this we mean a connected component of the complement of the union of all proper rational subspaces generated by subsets of $X$.

In this section we are going to discuss the fact that every element $f \in \mathcal{F}(X)$ coincides with a quasi-polynomial on the sets $(\tau-B(X)) \cap \Lambda$ as $\tau$ varies over all topes (we simply say $f$ is a quasi-polynomial on $\tau-B(X)$ ).

Definition 9.14 Let $\tau$ be a tope and $\underline{r}$ be a proper rational subspace. We say that a regular face $F_{\underline{r}}$ for $X \backslash \underline{r}$ is non-positive on $\tau$ if there exists $u_{\underline{r}} \in F_{\underline{r}}$ and $x_{0} \in \tau$ such that $\left\langle u_{r}, x_{0}\right\rangle<0$.

Given $x_{0} \in \tau$, it is always possible to choose a regular face $F_{r} \subset \underline{r}^{\perp}$ for $X \backslash \underline{r}$ such that $x_{0}$ is negative on some vector $u_{\underline{r}} \in F_{\underline{r}}$, since the projection of $x_{0}$ on $V / \underline{r}$ is not zero. 
C· PROCESI**

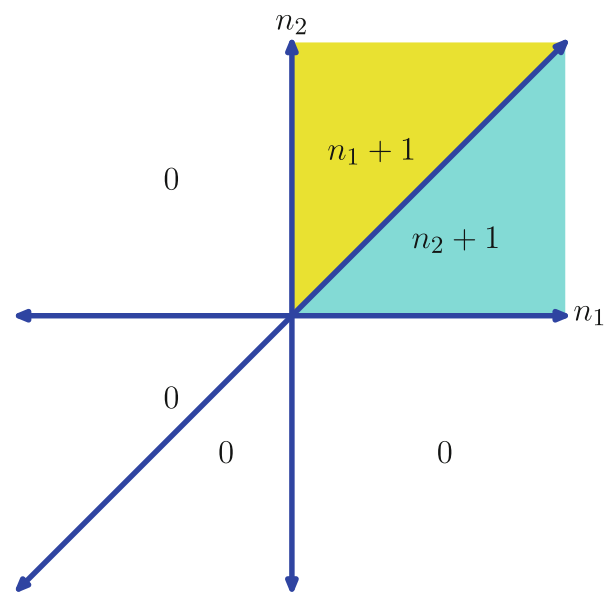

Fig. 6 The partition function of $X:=(a, b, c)$

Definition 9.15 Let $\mathbf{F}=\left\{F_{\underline{r}}\right\}$ be a $X$-regular collection. We shall say that $\mathbf{F}$ is nonpositive on $\tau$ if each $F_{r}$ is non-positive on $\tau$.

Let $f \in \mathcal{F}(X)$ and let $f=\sum f_{r}$ be the $\mathbf{F}$ decomposition of $f$.

The following result is quite similar to Paradan's localization theorem [29].

Theorem 19 (Localization theorem) Let $\tau$ be a tope. Let $\mathbf{F}=\left\{F_{\underline{r}}\right\}$ be a $X$-regular collection non-positive on $\tau$.

The component $f_{V}$ of the $\mathbf{F}$ decomposition $f=\sum_{r \in S_{X}} f_{\underline{r}}$ is a quasi-polynomial function in $D M(X)$ such that $f=f_{V}$ on $(\tau-B(X)) \bar{\cap} \Lambda$.

Example 9.16 Figure 6 describes the partition function $P_{X}$ for $X:=(a, b, c)$ with $a:=\omega_{1}, b:=\omega_{2}, c:=\omega_{1}+\omega_{2}$ in the lattice $\Lambda:=\mathbb{Z} \omega_{1} \oplus \mathbb{Z} \omega_{2}$. Figure 7 describes the $\mathbf{F}$ decomposition relative to the tope containing $x_{0}$. Notice how the choice of $\mathbf{F}$ has the effect of pushing the supports of the elements $f_{\underline{r}}(\underline{r} \neq V)$ away from $\tau$.

A quasi-polynomial is completely determined by the values that it takes on $(\tau-B(X)) \cap \Lambda$. Thus $f_{V}$ is independent on the construction so:

Definition 9.17 We shall denote by $f^{\tau}$ the quasi-polynomial coinciding with $f$ on $(\tau-B(X)) \cap \Lambda$.

The open subsets $\tau-B(X)$ cover $V$, when $\tau$ runs over the topes of $V$ (with possible overlapping). Thus the element $f \in \mathcal{F}(X)$ is entirely determined by the quasi-polynomials $f^{\tau}$.

If $f \in \mathcal{F}(X)$, the element $\nabla_{X \backslash \underline{r}} f$ is in $\mathcal{F}(X \cap \underline{r})$ and coincides with a quasi-polynomial $\left(\nabla_{X \backslash r} f\right)^{\tau} \in D M(X \cap \underline{r})$ on each tope $\tau$ for the system $X \cap \underline{r}$.

Theorem 20 Let $\beta \in V$ be generic with respect to all the rational subspaces $\underline{r}$. Let $F_{\underline{r}}^{\beta}$ be the unique regular face for $X \backslash \underline{r}$ containing $p_{\underline{r}^{\perp}} \beta$. 

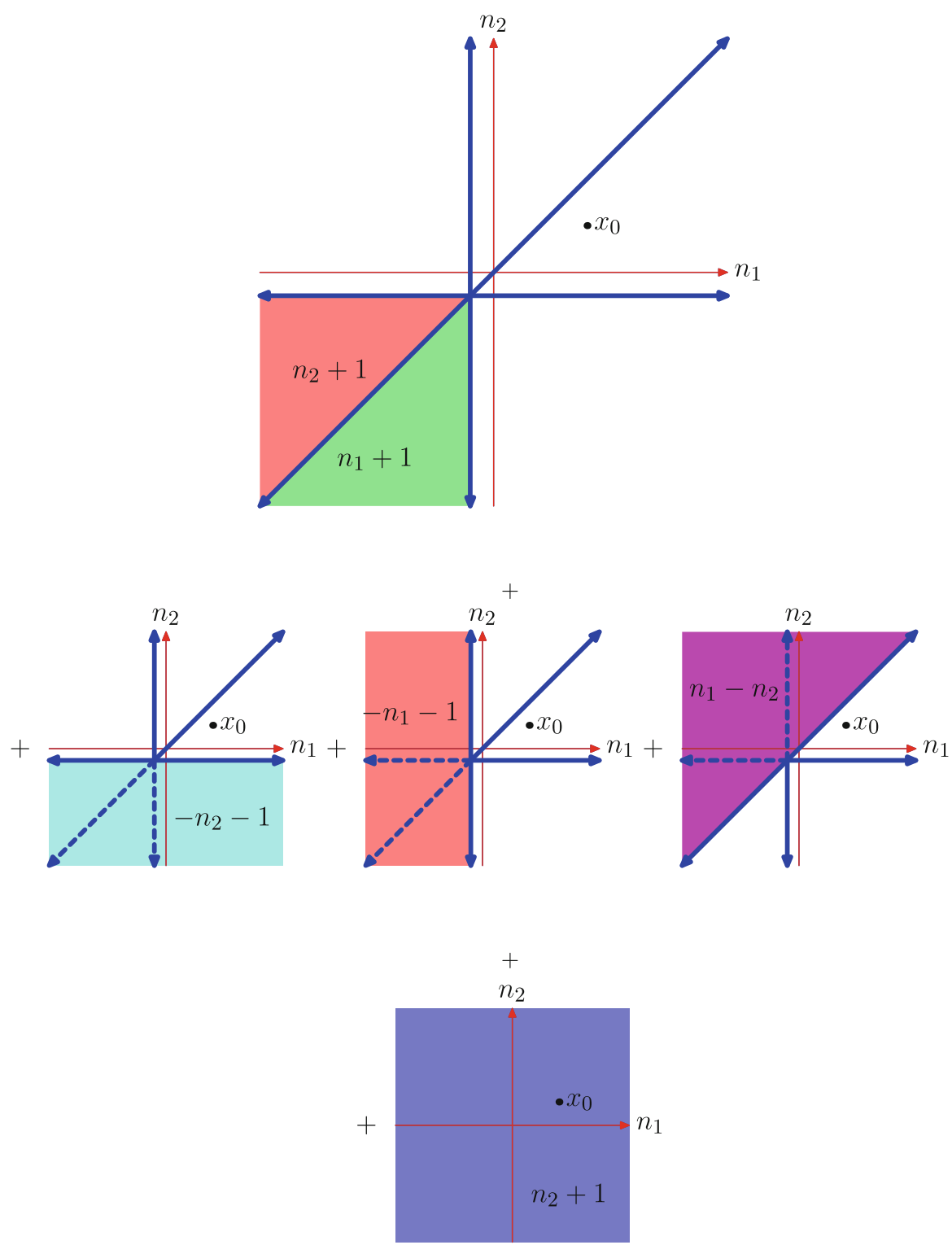

Fig. 7 F decomposition of the partition function of $X:=(a, b, c)$ for $\mathbf{F}$ non-positive on $\tau$

\section{Then}

$$
f=\sum_{\underline{r} \in S_{X}} P_{X \backslash \underline{\underline{r}}}^{-F_{\underline{r}}^{\beta}} *\left(\nabla_{X \backslash \underline{r}} f\right)^{\tau\left(p_{\underline{\underline{r}}} \beta\right)}
$$



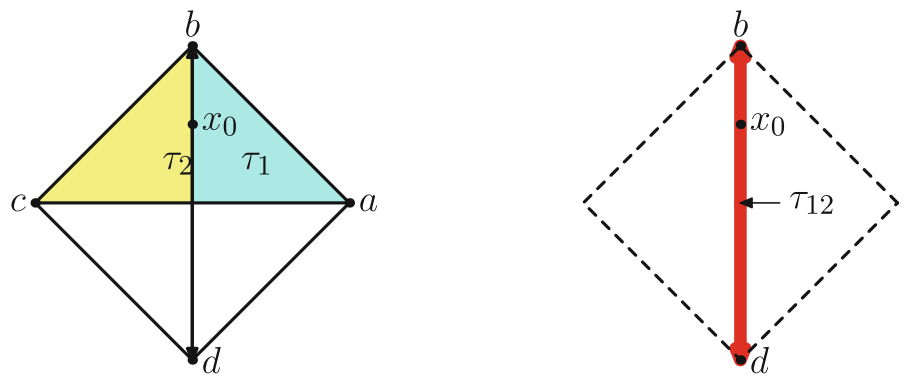

Fig. 8 Two adjacent topes of $X:=(a, b, c, d)$

9.17.1. Wall crossing formula. We first describe a general formula of how the functions $f^{\tau}$ change when crossing a wall. This formula implies that the partition function $P_{X}$ is a quasi-polynomial on $\Omega-B(X)$, where $\Omega$ is a big cell.

Let $H$ be a rational hyperplane and $u \in H^{\perp}$ a non zero element. Then the two open faces in $H^{\perp}$ are half-lines $F_{H}=\mathbf{R}_{>0} u$ and $-F_{H}$. If $q \in D M(X \cap H)$, then $w:=\left(P_{X \backslash H}^{F_{H}}-\mathcal{T}_{X \backslash H}^{-F_{H}}\right) * q$ is an element of $D M(X)$.

Remark 9.18 In [11], a one-dimensional residue formula is given for $w$ allowing us to compute it.

Assume that $\tau_{1}, \tau_{2}$ are two adjacent topes, namely $\bar{\tau}_{1} \cap \bar{\tau}_{2}$ spans a hyperplane $H$. The hyperplane $H$ is a rational subspace. Let $\tau_{12}$ be the unique tope for $X \cap H$ such that $\bar{\tau}_{1} \cap \bar{\tau}_{2} \subset \overline{\tau_{12}}$.

Example 9.19 Let $C$ be the cone generated by the vectors $a:=\omega_{3}+\omega_{1}, b:=\omega_{3}+\omega_{2}$, $c:=\omega_{3}-\omega_{1}, d:=\omega_{3}-\omega_{2}$ in a 3-dimensional space $V:=\mathbb{R} \omega_{1} \oplus \mathbb{R} \omega_{2} \oplus \mathbb{R} \omega_{3}$. Figure 8 represents the section of $C$ cut by the affine hyperplane containing $a, b, c, d$. We consider $X:=(a, b, c, d)$.

On the left of the picture we show the intersection of $C$ with the two topes $\tau_{1}, \tau_{2}$ adjacent along the hyperplane $H$ generated by $b, d$ and, on the right, that with the tope $\tau_{12}$. The list $X \cap H$ is $[b, d]$. The closure of the tope $\tau_{12}$ is "twice bigger" than $\bar{\tau}_{1} \cap \bar{\tau}_{2}$.

Let $f \in \mathcal{F}(X)$. The function $\nabla_{X \backslash H} f$ is an element of $\mathcal{F}(H \cap X)$, thus there exists a quasi-polynomial $\left(\nabla_{X \backslash H} f\right)^{\tau_{12}}$ on $H$ such that $\nabla_{X \backslash H} f$ agrees with $\left(\nabla_{X \backslash H} f\right)^{\tau_{12}}$ on $\tau_{12}$.

Theorem 21 Let $\tau_{1}, \tau_{2}, H, \tau_{12}$ be as before and $f \in \mathcal{F}(X)$. Let $F_{H}$ be the half line in $H^{\perp}$ positive on $\tau_{1}$. Then

$$
f^{\tau_{1}}-f^{\tau_{2}}=\left(P_{X \backslash H}^{F_{H}}-P_{X \backslash H}^{-F_{H}}\right) *\left(\nabla_{X \backslash H} f\right)^{\tau_{12}} .
$$

In the case in which $f=P_{X}$ we deduce Paradan's formula [28, Theorem 5.2]

$$
P_{X}^{\tau_{1}}-P_{X}^{\tau_{2}}=\left(P_{X \backslash H}^{F_{H}}-P_{X \backslash H}^{-F_{H}}\right) * P_{X \cap H}^{\tau_{12}} \cdot
$$


9.20 The partition function

We assume that $C(X)$ is a pointed cone. Let us now consider a big cell $\Omega$. Given a big cell $\Omega$, let $\tau_{1}, \ldots, \tau_{k}$ be all the topes contained in $\Omega$. Then:

$$
\Omega-B(X)=\cup_{i=1}^{k}\left(\tau_{i}-B(X)\right) .
$$

Now in order to prove the statement for big cells, we need to see what happens when we cross a wall between two adjacent topes by the previous formulas one has

Theorem 22 On $(\Omega-B(X)) \cap \Lambda$, the partition function $P_{X}$ agrees with a quasipolynomial $P_{X}^{\Omega} \in D M(X)$.

This theorem was proven [14] by Dahmen-Micchelli for topes, and by SzenesVergne [34] for cells. In many cases, the sets $\Omega-B(X)$ are the maximal domains of quasi-polynomiality for $\mathcal{T}_{X}$.

There is an important point of the theory of Dahmen-Micchelli that we point out.

Theorem 23 Let $\Omega$ be a big cell contained in $C(X)$. Then $P_{X}^{\Omega}$ is the unique element $f \in D M(X)$ such that $f(0)=1$ and $f(a)=0, \forall a \in \delta(\mathfrak{c} \mid X), a \neq 0$.

\section{Part 3. Index theory}

\section{The Atiyah-Singer index theorem}

\subsection{Equivariant $K$-theory}

We briefly review the notations for $K$-theory that we will use, for a systematic treatment see Atiyah [2] and Segal [32]. Let $G$ be a compact Lie group acting on a locally compact space $N$.

- One has the notion of the equivariant topological $K$-theory group $K_{G}^{0}(N)$.

- If $N$ is compact $K_{G}^{0}(N)$ is the Grothendieck ring of equivariant vector bundles.

- For a point $p$ it is the character ring $R[G]$ of $G$.

For $N$ locally compact set $\dot{N}=N \cup \infty$ be the one point compactification.

- One sets $K_{G}^{0}(N)$ to be the kernel of the restriction of $K_{G}^{0}(\dot{N})$ to $K_{G}^{0}(\infty)$

- $K_{G}^{0}(N)$ is then a contravariant functor for proper maps and covariant for open embeddings.

Remark 10.2 Notice that, when $G$ is abelian, the character ring $R[G]$ equals the group $\operatorname{ring} \mathbb{Z}[\hat{G}]$ of itd character group $\hat{G}$.

Representatives of the $K$-theory group $K_{G}^{0}(N)$ can be described in the following way.

Given two $G$-equivariant complex vector bundles $E^{0}, E^{1}$ on $N$ and a $G$-equivariant bundle map $f: E^{0} \rightarrow E^{1}$, the support $\operatorname{supp}(f)$ of $f$ is the set of points where $f_{x}: E_{x}^{0} \rightarrow E_{x}^{1}$ is not an isomorphism. 
A $G$-equivariant bundle map $f$ with compact support defines an element $[f]$ of $K_{G}^{0}(N)$. All elements can be described this way.

Let $f: E^{0} \rightarrow E^{1}$ and $g: F^{0} \rightarrow F^{1}$ be two $G$-equivariant bundle maps. Using $G$-invariant Hermitian metrics on the bundles $E^{i}, F^{i}$ we can define:

$$
f \odot g: E^{0} \otimes F^{0} \oplus E^{1} \otimes F^{1} \rightarrow E^{1} \otimes F^{0} \oplus E^{0} \otimes F^{1}
$$

by

$$
f \odot g:=\left(\begin{array}{cc}
f \otimes 1 & -1 \otimes g^{*} \\
1 \otimes g & f^{*} \otimes 1
\end{array}\right)
$$

The support of $f \odot g$ is the intersection of the supports of $f, g$ thus $f \odot g$ induces an element in $K_{G}^{0}(N)$ as soon as one of the two $f, g$ has compact support.

In particular this defines a product $[f][g]:=[f \odot g]$ on $K_{G}^{0}(N)$.

If $N=\mathrm{pt}$ is a point, $K_{G}^{0}(\mathrm{pt})$ is isomorphic to the Grothendieck ring $R(G)$ of finite dimensional representations of $G$.

In general take the projection $\pi: N \rightarrow \mathrm{pt}$, given $\tau \in R(G)$ and $\sigma \in K_{G}^{0}(N)$, we have that $\left[\pi^{*}(\tau) \odot \sigma\right] \in K_{G}^{0}(N)$ and this gives a $R(G)$ module structure to $K_{G}^{0}(N)$.

10.2.1. Clifford action. Let $W$ be a Hermitian vector space and let $E=\bigwedge W$.

For $w \in W$, consider the exterior multiplication $m(w): E \rightarrow E$ and the Clifford action

$$
c(w)=m(w)-m(w)^{*}, \quad m(w)(\omega):=w \wedge \omega
$$

of $W$ on $\bigwedge W$.

We have $c(w)^{2}=-\|w\|^{2}$, so that $c(w)$ is an isomorphism, if $w \neq 0$. We shall use this as follows:

10.2.2. Bott symbol and Thom isomorphism. If $p: W \rightarrow M$ is a $G$-equivariant complex vector bundle over a $G$-space $M$, the fiberwise Clifford action $c\left(w_{x}\right)$ : $\bigwedge^{\text {even }} W_{x} \rightarrow \bigwedge^{\text {odd }} W_{x}$ defines a morphism $\mathbf{c}_{W}: p^{*} \bigwedge^{\text {even }} W \rightarrow p^{*} \bigwedge^{\text {odd }} W$ of vector bundles over $W$, called the Bott symbol.

Take a bundle map $f: E \rightarrow F$ of complex equivariant vector bundles on $M$ which is an isomorphism outside a compact set, and denote still by $f$ its pull back $f: p^{*} E \rightarrow p^{*} F$. Then $f \odot \mathbf{c}_{W}$ is a bundle map of bundles over $W$, which is an isomorphism outside the support of $f$ embedded in $W$ via the zero section. We set

Thom isomorphism $\quad C_{W}: K_{G}^{0}(M) \rightarrow K_{G}^{0}(W), \quad C_{W}([f])=\left[f \odot \mathbf{c}_{W}\right]$.

The map $[f] \mapsto C_{W}([f])=\left[f \odot \mathbf{c}_{W}\right]$ is the Thom isomorphism between $K_{G}^{0} M$ and $K_{G}^{0} W$. 
10.2.3. Bott periodicity. The Bott periodicity is the Thom isomorphism when $W=M \times \mathbb{C}=M \times \mathbb{R}^{2}$. We then define inductively the groups

$$
K_{G}^{i+1}(N):=K_{G}^{i}(N \times \mathbb{R})
$$

One has $K_{G}^{0}(\mathbb{R})=K_{G}^{1}(\mathrm{pt})=0$.

There is a natural isomorphism $K_{G}^{i}(N) \rightarrow K_{G}^{i+2}(N)=K_{G}^{i}\left(N \times \mathbb{R}^{2}\right)$ given by Bott periodicity.

10.2.4. A long exact sequence. If $F$ is a $G$-invariant closed subset of $N$, denote by $i: F \rightarrow N$ the closed embedding and $j: N \backslash F \rightarrow N$ the open embedding. There is a long exact sequence of $R(G)$ modules:

$$
\cdots \rightarrow K_{G}^{i}(N \backslash F) \stackrel{j_{*}}{\rightarrow} K_{G}^{i}(N) \stackrel{i^{*}}{\rightarrow} K_{G}^{i}(F) \stackrel{\delta}{\rightarrow} K_{G}^{i+1}(N \backslash F) \rightarrow \cdots
$$

\subsection{Transversally elliptic operators}

Consider a manifold $M$ with an action of a compact Lie group $G$. We then have the notion of transversally elliptic operator between two equivariant complex vector bundles $E, F$ on $M$.

Such an operator is a pseudo-differential operator $A: \Lambda(M, E) \rightarrow \Lambda(M, F)$ from the space $\Lambda(M, E)$ of smooth sections of $E$ to the space $\Lambda(M, F)$ of smooth sections of $F$, which commutes with the action of $G$, is elliptic in the directions transversal to the orbits of $G$ and is "trivial" at infinity.

Let $T^{*} M$ denote the cotangent bundle of $M$ and $p: T^{*} M \rightarrow M$ the canonical projection.

Definition 10.4 By a symbol, one means a smooth section on $T^{*} M$ of the bundle $\operatorname{hom}\left(p^{*}(E), p^{*}(F)\right)$ : in other words, for each point $(x, \xi), x \in M, \xi \in T_{x}^{*} M$, we have a linear map $\sigma(x, \xi): E_{x} \rightarrow F_{x}$.

Inside $T^{*} M$, there is a special closed subset denoted by $T_{G}^{*} M$ (the zero set of the moment map cf. Sect. 13.3). Its fiber over a point $x \in M$ is formed by all the cotangent vectors $\xi \in T_{x}^{*} M$ which vanish on the tangent space to the orbit of $x$ under $G$, in the point $x$. Thus each fiber $\left(T_{G}^{*} M\right)_{x}$ is a linear subspace of $T_{x}^{*} M$. In general the dimension of $\left(T_{G}^{*} M\right)_{x}$ is not constant and this space is not a vector bundle.

Assume first that $M$ is a compact manifold. To the pseudo-differential operator $A$, one associates its principal symbol $\sigma_{p}$ which is defined outside the zero section of $T^{*} M$. The operator $A$ is said to be $G$-transversally elliptic if its principal symbol $\sigma_{p}(x, \xi)$ is invertible for all $(x, \xi) \in T_{G}^{*} M$ such that $\xi \neq 0$.

Using a $G$-invariant function $\chi$ on $T^{*} M$ identically equal to 1 in a neighborhood of $M$ and compactly supported, then $\sigma(x, \xi):=(1-\chi(x, \xi)) \sigma_{p}(x, \xi)$ is defined on the whole space $T^{*} M$. Furthermore $\sigma(x, \xi)$ restricted to $T_{G}^{*} M$ is an isomorphism outside a compact $G$-invariant subset of $T_{G}^{*} M$.

Thus, by restriction to $T_{G}^{*} M$, the symbol $\sigma$ defines a $K$-theory class $[\sigma]$ in the topological equivariant $K$-theory group $K_{G}^{0}\left(T_{G}^{*} M\right)$. 
This class does not depend of the choice of $\chi$. We still say that this class $\sigma$ is the symbol of $A$.

Let $\hat{G}$ be the set of equivalence classes of finite dimensional irreducible representations of $G$, and let $\mathcal{C}[\hat{G}]$ be the group of $\mathbb{Z}$-valued functions on $\hat{G}$. Let $\chi_{\tau}(g)=\operatorname{Tr}(\tau(g))$ be the character of the representation $\tau \in \hat{G}$ of $G$. We associate to an element $f \in \mathcal{C}[\hat{G}]$ a formal (virtual) character $\Theta(f)=\sum_{\tau} f(\tau) \chi_{\tau}$, that is a formal combination of the characters $\chi_{\tau}$ with multiplicities $f(\tau) \in \mathbb{Z}$. When $f(\tau)$ satisfies certain moderate growth conditions, then the series $\Theta(f)(g)=\sum_{\tau} f(\tau) \chi_{\tau}(g)$ converges, in the distributional sense, to a generalized function on $G$.

The index map associates to a transversally elliptic operator $A$ an element of $\mathcal{C}[\hat{G}]$ constructed as follows. For every $\tau \in \hat{G}$, the $\operatorname{space}_{\operatorname{hom}_{G}}(\tau, \operatorname{ker}(A))$ is finite dimensional of dimension $m(\tau, A)$. Thus $m(\tau, A)$ is the multiplicity of $\tau$ in the space $\operatorname{ker}(A)$ of smooth solutions of $A$. We choose a $G$-invariant metric on $M$ and $G$-invariant Hermitian structures on $E, F$. Then $A^{*}: \Lambda(M, F) \rightarrow \Lambda(M, E)$ is also transversally elliptic.

Definition 10.5 The index multiplicity of the pseudo-differential operator $A$ is the function $\operatorname{ind}_{m}(A) \in \mathcal{C}[\hat{G}]$ defined by

$$
\operatorname{ind}_{m}(A)(\tau):=m(\tau, A)-m\left(\tau, A^{*}\right) .
$$

It follows also from Atiyah-Singer [1] that the series $\sum_{\tau} m(\tau, A) \chi_{\tau}(g)$ defines a generalized function on $G$. Thus we may also associate to $A$ the generalized function

$$
\operatorname{ind}(A)(g)=\sum_{\tau} i n d_{m}(A)(\tau) \chi_{\tau}(g)
$$

on $G$ with integral Fourier coefficients. One of the main points in the index theory consists in showing that the index factors through the symbols and defines a homomorphism of $R(G)$ modules from $K_{G, c}^{0}\left(T_{G}^{*} M\right)$ to $\mathcal{C}[\hat{G}]$.

If $j: U \rightarrow M$ is an open $G$-invariant set of a compact $G$ manifold $M$, we still denote by $j$ the corresponding open embedding from $T_{G}^{*} U$ to $T_{G}^{*} M$. Then $j_{*}$ defines a map from $K_{G}^{0}\left(T_{G}^{*} U\right)$ to $K_{G, c}^{0}\left(T_{G}^{*} M\right)$. The index of $\sigma \in K_{G}^{0}\left(T_{G}^{*} U\right)$ is defined to be the index of $j_{*}(\sigma)$. The excision property of the index shows that this is independent of the choice of the open embedding $j$ and thus allows us to define the index map also for manifolds which can be embedded as open sets of compact ones.

In particular, if $V$ is a vector space with a linear action of a compact group $G$, then $V$ is diffeomorphic to the sphere, minus a point. Thus we can define the index of any $\sigma \in K_{G}^{0}\left(T_{G}^{*} V\right)$. More generally, if $U$ is an open $G$-invariant subset of a vector bundle on a compact manifold, we can define the index of $\sigma \in K_{G}^{0}\left(T_{G}^{*} U\right)$.

The problem of computing the index can be reduced, at least theoretically, to the case in which $G$ is a torus. For a given compact manifold $M$, one embeds $M$ into a linear representation and then is reduced to perform the computations in the representation. 


\subsubsection{Some properties of the index map}

(i) Any element $\sigma \in K_{G}^{0}\left(T_{G}^{*} M\right)$ arises from the restriction to $T_{G}^{*} M$ of a $G$ - bundle morphism $\sigma(x, \xi): E_{x} \rightarrow F_{x}$, such that $\operatorname{supp}(\sigma) \cap T_{G}^{*} M$ is a compact set. Here $E, F$ are $G$-equivariant complex vector bundles over $M$.

(ii) If $j: \mathcal{U} \rightarrow M$ is an open embedding, the map $j_{*}: K_{G}^{0}\left(T_{G}^{*} \mathcal{U}\right) \rightarrow K_{G}^{0}\left(T_{G}^{*} M\right)$ is compatible with the index.

(iii) Let $W$ be a real vector space with a linear representation of $G$ and $W^{\prime}$ be the dual vector space. We identify $T W=W \times W$ with $W_{\mathbb{C}}$ by $(v, w) \rightarrow v+i w$. Furthermore, we identify $T W=W \times W$ with $T^{*} W=W \times W^{\prime}$ using an Euclidean structure on $W$. Thus the Bott symbol $c_{W_{\mathbb{C}}}(v+i \xi)$ acting on $\wedge W_{\mathbb{C}}$ defines a $G$-equivariant elliptic symbol on $W$. Its $G$-equivariant index is identically equal to 1 .

Let $i: N \rightarrow M$ be an injection of $G$-manifolds, this factors through a tubular neighborhood $j: \mathcal{U} \rightarrow M$. Using a similar construction we obtain a map $i_{!}: K_{G}^{0}\left(T_{G}^{*} N\right) \rightarrow K_{G}^{0}\left(T_{G}^{*}(\mathcal{U})\right)$ given at the level of symbols by $\sigma \mapsto \sigma \odot c_{W_{\mathbb{C}}}$. The index of $\sigma$ is equal to the index of $i_{!} \sigma$.

(iv) In case $M=N \times \mathbb{R}$ with the trivial action on $\mathbb{R}, T_{G}^{*}(N \times \mathbb{R})=T_{G}^{*} N \times T^{*} \mathbb{R}$ and thus $i$ ! is an isomorphism by Bott periodicity.

(v) Let $H$ be a closed subgroup of $G$. Then there is a surjective map $\hat{G} \rightarrow \hat{H}$ induced by the restriction of characters. The dual map induces an injection $\operatorname{Ind}_{H}^{G}: \mathcal{C}[\hat{H}] \rightarrow \mathcal{C}[\hat{G}]$.

Let $M$ be a space with $H$ action (open subset a compact $H$-manifold), and let $N:=G \times{ }_{H} M$ be the $G$ space with typical fiber $M$ over $G / H$. It is easy to see that there is an isomorphism

$$
i_{H}^{G}: K_{H}^{i}\left(T_{H}^{*} M\right) \rightarrow K_{G}^{i}\left(T_{G}^{*} N\right)
$$

and, by $\left[1\right.$, Theorem 4.1], for any $\sigma \in K_{H}^{0}\left(T_{H}^{*} M\right)$,

$$
\operatorname{ind}_{m}\left(i_{H}^{G}(\sigma)\right)=\operatorname{Ind}_{H}^{G}\left(\operatorname{ind}_{m}(\sigma)\right) .
$$

We shall also need the following simple consequence of the previous facts:

Lemma 10.6 Let $G$ be a compact Lie group and $\chi: G \rightarrow S^{1}$ be a surjective character. Set $H:=\operatorname{ker} \chi$ be the kernel of $\chi$.

Take a manifold $M$ over which $G$ acts and consider the product $\mathbb{C}^{*} \times M$, with the action of $G$ on the first factor induced by $\chi$.

There is an isomorphism

$$
k: K_{H}^{i}\left(T_{H}^{*} M\right) \cong K_{G}^{i}\left(T_{G}^{*}\left(\mathbb{C}^{*} \times M\right)\right) .
$$

Moreover, if $\sigma \in K_{H}^{0}\left(T_{H}^{*} M\right)$, we have ind $d_{m}(k(\sigma))=\operatorname{Ind}_{H}^{G}\left(i n d_{m}(\sigma)\right)$.

Since $\mathbb{C}^{*}=S^{1} \times \mathbb{R}^{+}$, we get by iv) that the inclusion $i: S^{1} \times M \rightarrow \mathbb{C}^{*} \times M$ induces the isomorphism

$$
i_{!}: K_{G}^{i}\left(T_{G}^{*}\left(S^{1} \times M\right)\right) \rightarrow K_{G}^{i}\left(T_{G}^{*}\left(\mathbb{C}^{*} \times M\right)\right)
$$


which at the level of $K^{0}$ is compatible with the index.

On the other hand, the space $G \times{ }_{H} M$ identifies with $S^{1} \times M$ via the map $[g, m] \mapsto$ $[\chi(g), g \cdot m]$. So (27) gives us the isomorphism

$$
i_{H}^{G}: K_{H}^{i}\left(T_{H}^{*}(M)\right) \rightarrow K_{G}^{i}\left(T_{G}^{*}\left(S^{1} \times M\right)\right) .
$$

If $\sigma \in K_{H}^{0}\left(T_{H}^{*} M\right)$, then $i n d_{m}\left(i_{H}^{G}(\sigma)\right)=\operatorname{Ind}_{H}^{G}\left(i n d_{m}(\sigma)\right)$ by Formula (28).

Thus we can take $k:=i_{!} i_{H}^{G}$.

\section{- The tangential Cauchy-Riemann operator.}

Assume that the group $G$ is an abelian compact Lie group. An irreducible representation $a$ of $G$ is a one dimensional complex vector space $L_{a}$, where $G$ acts via a character $\chi_{a}: G \rightarrow S^{1}$, so that $\hat{G}$ is identified with the abelian group of characters, denoted by $\Lambda$.

Definition 10.7 Let $X$ be a finite list of elements of $\Lambda$. Define the complex vector space

$$
M_{X}:=\oplus_{a \in X} L_{a} .
$$

The space $M_{X}$ is a $G$-manifold and our goal is the determination of $K_{G}^{0}\left(T_{G}^{*} M_{X}\right)$. The basic tool that we shall use is the space of functions $D M^{(G)}(X)$ on $\Lambda$. This is defined as $D M(X)$ (and often denoted also $D M(X)$ ) but in this more general case of not necessarily connected $G$. The extension to non connected abelian groups is necessary in order to perform induction. In this case $\Lambda$ has a torsion subgroup $\Lambda_{t}$ and Theorem 13 gives the statement that $D M^{(G)}(X)$ is a free module over the group ring of $\Lambda_{t}$ or $\operatorname{rank} \delta(X)$.

With a given $G$-invariant Hermitian structure on $M$ let $S$ be the unit sphere of $M$. Let $\mathbb{P}(M)$ be the complex projective space of $M$. Consider on $S$ the differential operator $\delta$ acting on the pull back of the Dolbeault complex on the associated projective space $\mathbb{P}(M)$ using $\bar{\partial}+\bar{\partial}^{*}: \sum \Omega^{0,2 p} \rightarrow \sum \Omega^{0,2 p+1}$. Then $\delta$ is a $G$-transversally elliptic differential operator the tangential Cauchy-Riemann operator on $S$.

Indeed, using the Hermitian structure, identify $T^{*} S$ with its tangent bundle $T S \subset T M$, the subspace $H_{p}$ of $T_{p}^{*} S$ orthogonal to the line $\mathbb{R} J_{u} p$ is then identified to the complex subspace of $M$, orthogonal under the Hermitian form to $p$. We call it the horizontal cotangent space. The symbol of $\delta$ is $\sigma(p, \xi)=c\left(\xi^{1}\right)$ where $\xi^{1}$ is the projection of $\xi$ on $H_{p}$, and $c$ the Clifford action of $H_{p}$ on $\bigwedge H_{p}$. This morphism is invertible if $\xi^{1} \neq 0$. We have also $H_{p} \oplus \mathbb{R} \rho(u) p=T_{p}^{*} S$, as the eigenvalues of $-i u$ on $M$ are all positive. Thus we see that $\sigma(p, \xi)$ restricted to $T_{G}^{*} S$ is invertible outside the zero section.

The following formula is proven in [1] (Proposition 5.4).

Theorem 24 Let $M$ be provided with the complex structure $J_{u}$ and let $\delta$ be the tangential Cauchy-Riemann operator, on the unit sphere of $M$. Then

$$
\operatorname{index}(\delta)(g)=(-1)^{|X|} g^{a_{X}}\left(\Theta_{X}^{-F}(g)-\Theta_{X}^{F}(g)\right)
$$

where $a_{X}=\sum_{a \in X} a$. 
We recall briefly the proof. Let $S^{1}$ be the circle group acting by homotheties on $M$. We decompose solution spaces with respect to characters $t \mapsto t^{n}$ of $S^{1}$. The group $G$ acts on $\mathbb{P}(M)$ and on every line bundle $\mathcal{O}(n)$ on $\mathbb{P}(M)$. Thus the index as an index of $G \times S^{1}$ is the sum of the index of $G$ in the cohomology on $\mathbb{P}(M)$ of the line bundles $\mathcal{O}(n)$. Define $\chi_{n}(g)$ as the virtual character (as a representation of $G$ ) in the virtual finite dimensional vector space $\sum(-1)^{i} H^{0, i}(\mathbb{P}(M), \mathcal{O}(n))$. Then

$$
\operatorname{index}(\delta)(g)=\sum_{n \in \mathbb{Z}} \chi_{n}(g)
$$

Let us show that

$$
\begin{aligned}
& \sum_{n \geq 0} \chi_{n}(g)=(-1)^{|X|} g^{a_{X}} \Theta_{X}^{-F}(g), \\
& \sum_{n<0} \chi_{n}(g)=(-1)^{|X|+1} g^{a_{X}} \Theta_{X}^{F}(g) .
\end{aligned}
$$

For $n \geq 0, \mathcal{O}(n)$ has only 0 -cohomology and $H^{0,0}(\mathbb{P}(M), \mathcal{O}(n))$ is just the space of homogeneous polynomials on $M$ of degree $n$. So $\sum_{n=0}^{\infty} \chi_{n}$ is the character of the symmetric algebra $S\left[M^{*}\right]=\prod_{a \in X} S\left[L_{-a}\right]$. The function $\sum_{k=0}^{\infty} g^{-k a}$ is the character of the action of $G$ in $S\left[L_{-a}\right]$. The function $\Theta_{X}^{-F}$ is the product of the functions $-g^{-1} \sum_{k=0}^{\infty} g^{-k a}$. Thus we obtain Formula (31). On the other hand, if $n<0$, we have two cases. If $-|X|-1<n \leq-1$, then $H^{0, i}(\mathbb{P}(M), \mathcal{O}(n))=0$ for every $i$. Otherwise we apply Serre's duality and we obtain the second equality (32).

- Atiyah-Singer pushed symbol. We identify $T^{*} M$ with $M \times M$, using the Hermitian metric on $M$. Let $c(v): \bigwedge^{\text {even }} M \rightarrow \bigwedge^{\text {odd }} M$ be the Clifford action (24) of $M$ on $\bigwedge M$. Given, as before, a regular element $u$ in the Lie algebra of $G$ and letting $\rho(u)$ denote its infinitesimal action on $M$, we define

\section{Definition 10.8}

$$
A t_{u}(v, \xi)=c(\xi+\rho(u) v)
$$

The morphism $A t_{u}(v, \xi)$ is invertible except if $\xi+\rho(u) v=0$. If furthermore $\xi$ is in $T_{G}^{*} M, \xi$ is orthogonal to the tangent vector $\rho(u) v$. Thus the support of $A t_{u}(v, \xi)$ restricted to $T_{G}^{*} M$ is the unique point $v=0, \xi=0$ and $A t_{u}$ determines an element of $K_{G}^{0}\left(T_{G}^{*} M\right)$, which depends only of the open face $F$ of $u$ in the hyperplane arrangement dual to $X$. We denote it by $A t_{F}$. The index of $A t_{F}$ is computed in [1] (Theorem 8.1). In more detail, in the Appendix of [9], it is constructed an explicit $G$-transversally elliptic pseudo-differential operator $A$ on the product of the projective lines $\mathbb{P}\left(L_{a} \oplus \mathbb{C}\right)$.

If $j: M_{X} \rightarrow \prod_{a \in X} \mathbb{P}\left(L_{a} \oplus \mathbb{C}\right)$ is the natural open embedding, it is shown that $j_{*}\left(A t_{F}\right)$ is homotopic to the symbol of $A$. By definition, the index of $A t_{F}$ is that of $A$ and one has the explicit formula: 
Theorem 25 Let $M$ be provided with the complex structure $J_{u}$ and let $A_{F} \in$ $K_{G}^{0}\left(T_{G}^{*} M\right)$ be the "pushed" $\bar{\partial}$ symbol. Then

$$
\operatorname{index}\left(A t_{F}\right)(g)=(-1)^{|X|} g^{a_{X}} \Theta_{X}^{F}(g) \text {. }
$$

\section{The index for $M_{X}$}

This section contains the main results on the index for the space $M_{X}$, that is Theorems 28 and 29.

\section{$11.1 K$-theory}

Let $G$ be, as before, a compact abelian Lie group of dimension $s$ and $M_{X}:=\bigoplus_{a \in X} L_{a}$ as in (30). We assume that $X$ has rank $s$.

Given a vector $v \in M_{X}$, its support is the sublist of elements $a \in X$ such that $v$ has a non zero coordinate in the summand $L_{a}$.

If $Y$ is the support of $v$, an element $t$ of $G$ stabilizes $v$ if and only if $t^{a}=1$ for all $a \in Y$. If $Y$ spans a rational subspace of dimension $k$, the $G$-orbit of $v$ has dimension $k$.

For any rational subspace $\underline{r}$, we may consider the subspace $M_{\underline{r}}:=\bigoplus_{a \in X \cap \underline{r}} L_{a}$ of $M_{X}$. We set

$$
\begin{gathered}
M_{\leq i}:=\cup_{\underline{r} \in S_{X}^{(i)}} M_{\underline{r}}, \quad M_{\geq i}:=M_{X} \backslash M_{\leq i-1}, \\
F_{i}:=M_{\leq i} \backslash M_{\leq i-1}=M_{\geq i} \backslash M_{\geq i+1}=M_{\leq i} \cap M_{\geq i} .
\end{gathered}
$$

Notice that

$$
M_{X}=M_{\geq 0} \supset M_{\geq 1} \supset M_{\geq 2} \supset \cdots \supset M_{\geq s}:=M_{X}^{f} .
$$

The set $M_{\leq i}$ is the closed set of points in $M$ with the property that the orbit has dimension $\leq i$ while $M_{\geq i}$ is the open set of points in $M$ with the property that the orbit has dimension $\geq \bar{i}$. The set $F_{i}$ is open in $M_{\leq i}$ and closed in $M_{\geq i}$ and it is the set of points in $M$ whose orbit under $G$ has dimension exactly $i$. In particular, $F_{s}=M_{\geq s}=M_{X}^{f}$ is the open set of points in $M$ with finite stabilizer under the action of $G$, which plays a particular role.

Definition 11.2 Given a rational subspace $\underline{r}$, we denote by $G_{r}$ the subgroup of $G$ joint kernel of the elements in $\Lambda \cap \underline{r}$. The group $G_{\underline{r}}$ is a torus and acts trivially on $M_{\underline{r}}:=\bigoplus_{a \in X \cap \underline{r}} L_{a}$ inducing an action of $G / G_{\underline{r}}$.

We define the set $F(\underline{r})$ to be the open set of $M_{\underline{r}}$ where $G / G_{\underline{r}}$ acts with finite stabilizers.

In other words, the connected component of the stabilizer of an element of $F(\underline{r})$ is exactly the group $G_{\underline{r}}$. 
Remark 11.3 By definition of $G_{r}$, the set $F(\underline{r})$ is non-empty. The set $F_{i}$ is the disjoint union of the sets $F(\underline{r})$ as $\underline{r}$ runs over all rational subspaces of dimension $i$. Thus the space $M_{X}$ is the disjoint union of the locally closed strata $F(\underline{r})$.

We now analyze the equivariant $K$ theory of $T_{G}^{*} M_{X}^{f}$.

Let $a \in X$ be an element of infinite order so that the homomorphism $g^{a}: G \rightarrow S^{1}$ is surjective. Set $Z:=X \backslash\{a\}$ and $G_{a}:=\operatorname{ker} g^{a}$. Denote by $\tilde{Z}$ the list of the restrictions to $G_{a}$ of the elements of $Z$. For $v \in M_{X}$, denote by $v_{a} \in \mathbb{C}$ its coordinate in $L_{a}$ with respect to a choice of a basis of the one dimensional vector space $L_{a}$.

The set $M_{Z}^{f}:=\left\{v \in M_{X}^{f} \mid v_{a}=0\right\}$ is closed in $M_{X}^{f}$. Denote by $i: M_{Z}^{f} \rightarrow M_{X}^{f}$ the closed embedding and by $j: M_{X}^{f} \backslash M_{Z}^{f} \rightarrow M_{X}^{f}$ the open embedding of the complement.

Lemma 11.4 There exists an isomorphism

$$
k: K_{G_{a}}^{i}\left(T_{G_{a}}^{*} M_{\tilde{Z}}^{f}\right) \rightarrow K_{G}^{i}\left(T_{G}^{*}\left(M_{X}^{f} \backslash M_{Z}^{f}\right)\right) .
$$

If $\sigma \in K_{G_{a}}^{0}\left(T_{G_{a}}^{*} M_{\tilde{Z}}^{f}\right)$, we have ind $d_{m}(k(\sigma))=\operatorname{Ind}_{G_{a}}^{G}\left(i n d_{m}(\sigma)\right)$.

Take an element $\left(v_{a}, w\right) \in L_{a} \times M_{Z}$ with $v_{a} \neq 0$, its stabilizer in $G$ is the subgroup of $G_{a}$ stabilizing $w$, therefore the space $M_{X}^{f} \backslash M_{Z}^{f}$ is isomorphic to $\mathbb{C}^{*} \times M_{\tilde{Z}}^{f}$. Thus we are in the setting of Lemma 10.6.

For a real vector space $W$, we shall denote by $W^{\prime}$ its dual. Consider the projection $p: T_{G}^{*} M_{X}^{f} \rightarrow M_{X}^{f}$. Then $p^{-1} M_{Z}^{f}$ is a closed subset of $T_{G}^{*} M_{X}^{f} \subset M_{X}^{f} \times M_{X}^{\prime}$ and $T_{G}^{*} M_{X}^{f} \backslash p^{-1} M_{X}^{f}$ is equal to $T_{G}^{*}\left(M_{X}^{f} \backslash M_{Z}^{f}\right)$. We use the same notations $i, j$ also in this setting for the closed and open embedding associated. Remark the following fact.

Lemma 11.5 (i) We have $p^{-1} M_{Z}^{f}=T_{G}^{*} M_{Z}^{f} \times L_{a}^{\prime}$.

(ii) We have an isomorphism $C_{a}: K_{G}^{i}\left(T_{G}^{*} M_{Z}^{f}\right) \rightarrow K_{G}^{i}\left(p^{-1} M_{Z}^{f}\right)$.

The first assertion is immediate to verify. The second follows from the first and Thom isomorphism. The first theorem (see [20] for a proof) is:

Theorem $26 \quad$ (i) $K_{G}^{1}\left(T_{G}^{*} M_{X}^{f}\right)=0$.

(ii) If $a \in X$ has infinite order, there is a short exact sequence:

$$
0 \rightarrow K_{G_{a}}^{0}\left(T_{G_{a}}^{*} M_{\tilde{Z}}^{f}\right) \stackrel{j_{*} k}{\rightarrow} K_{G}^{0}\left(T_{G}^{*} M_{X}^{f}\right) \stackrel{C_{a}^{-1} i^{*}}{\rightarrow} K_{G}^{0}\left(T_{G}^{*} M_{Z}^{f}\right) \rightarrow 0
$$

Choose $0 \leq i \leq s$. We pass now to study the $G$-invariant open subspace $M_{\geq i}$ of $M$. The set $M_{\geq i+1}$ is open in $M_{\geq i}$ with complement the space $F_{i}$ disjoint union of the spaces $F(\underline{r})$ with $\underline{r} \in S_{X}^{(i)}$. Denote by $\tilde{T}_{G}^{*} F_{i}$ the restriction of $T_{G}^{*} M$ to $F_{i}$, disjoint union of the spaces $\tilde{T}_{G}^{*} F(\underline{r})$. Denote $j: M_{\geq i+1} \rightarrow M_{\geq i}$ the open inclusion and $e: \tilde{T}_{G}^{*} F_{i} \rightarrow T_{G}^{*} M_{\geq i}$ the closed embedding. Let $C_{i}$ be the Thom isomorphism from $K_{G}^{0}\left(T_{G}^{*} F_{i}\right)$ to $K_{G}^{0}\left(\tilde{T}_{G}^{*} F_{i}\right)$ direct sum of the Thom isomorphisms $C_{\underline{r}}$. 
Theorem 27 For each $0 \leq i \leq s-1$,

(i) $K_{G}^{1}\left(T_{G}^{*} M_{\geq i}\right)=0$.

(ii) The following sequence is exact

$$
0 \rightarrow K_{G}^{0}\left(T_{G}^{*} M_{\geq i+1}\right) \stackrel{j_{*}}{\rightarrow} K_{G}^{0}\left(T_{G}^{*} M_{\geq i}\right) \stackrel{C_{i}^{-1} e^{*}}{\rightarrow} K_{G}^{0}\left(T_{G}^{*} F_{i}\right) \rightarrow 0 .
$$

Since $M_{\geq s}=M_{X}^{f}$, we can assume by induction on $s-i$ that (i) holds for each $j>i$. Also by Theorem 26 (i) we get that $K_{G}^{1}\left(T_{G}^{*} F_{i}\right)=0$ for each $0 \leq i \leq s-1$. Using this both statements follow immediately from the long exact sequence of equivariant $K$-theory.

Remark 11.6 The fact that the sequence (36) is exact is proved in [1] using a splitting.

11.6.1. Two commutative diagrams Let $N$ be a complex representation space for $G$. Recalling the structure of $R(G)$ module of the equivariant $K$-theory, the multiplication by the difference $\bigwedge^{\text {even }} N-\bigwedge^{\text {odd }} N$ will be denoted by $\bigwedge_{-1} N \otimes-$. This is by definition the action of the element $\operatorname{det}_{N}(1-g) \in R(G)$ on the equivariant $K$-theory.

Lemma 11.7 Take a sublist $Y$ in $X$ and decompose $M_{X}=M_{X \backslash Y} \oplus M_{Y}$. Let $U$ be an open $G$-invariant set contained in $M_{X \backslash Y} \times\left(M_{Y} \backslash\{0\}\right)$. Then if $\sigma \in K_{G}^{i}(U)$ or $\sigma \in K_{G}^{i}\left(T_{G}^{*} U\right)$, we have $\bigwedge_{-1} M_{Y} \otimes \sigma=0$.

We give the proof for $U$, the case of $T_{G}^{*} U$ being identical.

Take $v \in U$ and decompose it as $v=v_{X \backslash Y}+v_{Y}$ with $v_{X \backslash Y} \in M_{X \backslash Y}$ and $v_{Y} \in M_{Y}$. The component $v_{Y}$ is not zero by assumption. Consider the complex $G$-equivariant vector bundle $V_{Y}=U \times M_{Y}$ on $U$. Set now $E^{+}:=U \times \bigwedge^{\text {even }} M_{Y}, E^{-}=U \times \bigwedge^{\text {odd }} M_{Y}$. Choosing an Hermitian metric on $M_{Y}$, for every $u \in M_{Y}$, we get the Clifford action $c(u): \bigwedge^{\text {even }} M_{Y} \rightarrow \bigwedge^{\text {odd }} M_{Y}$ of $M_{Y}$ on $\bigwedge M_{Y}$, which is an isomorphism as soon as $u \neq 0$.

Going back to our bundles $E^{+}, E^{-}$, for every $\epsilon \in[0,1]$, define the bundle map $\mathbf{c}_{\epsilon}: E^{+} \rightarrow E^{-}$by

$$
\mathbf{c}_{\epsilon}(v, \omega)=\left(v, \epsilon c\left(v_{Y}\right) \omega\right) \text {. }
$$

If $\sigma \in K_{G}^{0}(U)$, the element $\bigwedge_{-1} M_{Y} \otimes \sigma \in K_{G}^{0}(U)$ is represented by the morphism $\mathbf{c}_{\mathbf{0}} \odot \sigma$, homotopic to $\mathbf{c}_{\mathbf{1}} \odot \sigma$. This last bundle map is an isomorphism since $v_{Y} \neq 0$ on $U$. This implies that $\bigwedge_{-1} M_{Y} \otimes \sigma=0$.

We apply this to the open set $M_{X}^{f}$ where $G$ acts with finite stabilizers. If $Y$ is a cocircuit, $M_{X \backslash Y} \cap M_{X}^{f}=\emptyset$, thus $\bigwedge_{-1} M_{Y} \otimes \sigma=0$ for all $\sigma \in K^{0}\left(T_{G}^{*} M_{X}^{f}\right)$. As the index map is a $R(G)$ module map, this implies that for cocircuit $Y$, the generalized function index $(\sigma)(g)$ on $G$ satisfies the equation $\prod_{a \in Y}\left(1-g^{a}\right) \operatorname{index}(\sigma)(g)=0$. The function $\operatorname{ind}_{m}(\sigma)$ on $\hat{G}$ is the Fourier transform of the function index $(\sigma)$. It follows that $\nabla_{Y} i n d_{m}(\sigma)=0$.

Thus we obtain 
Corollary 11.8 The multiplicity index map ind $d_{m}$ maps $K_{G}^{0}\left(T_{G}^{*} M_{X}^{f}\right)$ to the space $\operatorname{DM}(X)$.

More generally the same argument shows that

Corollary 11.9 Choose $0 \leq i \leq s$. If $\sigma \in K_{G}^{0}\left(T_{G}^{*} M_{\geq i}\right)$ and $\underline{t}$ is a rational subspace of dimension strictly less than $i$, then $\bigwedge_{-1} M_{X \backslash \underline{t}} \otimes \sigma=0$.

Let us now split $X=A \cup B$ and $M_{X}=M_{A} \oplus M_{B}$. Let $p: T_{G}^{*} M_{X} \rightarrow M_{X}$ be the projection and consider $\tilde{T}_{G}^{*} M_{A}:=p^{-1} M_{A}$. We have $\tilde{T}_{G}^{*} M_{A}=T_{G}^{*} M_{A} \times M_{B}^{\prime}$. In particular, we get a Thom isomorphism

$$
C_{M_{B}^{\prime}}: K_{G}^{0}\left(T_{G}^{*} M_{A}\right) \rightarrow K_{G}^{0}\left(\tilde{T}_{G}^{*} M_{A}\right) \cong K_{G}^{0}\left(T_{G}^{*} M_{A} \times M_{B}^{\prime}\right) .
$$

Denote by $i$ the closed inclusion $M_{A} \rightarrow M_{X}$, and, by abuse of notation, also the inclusion $\tilde{T}_{G}^{*} M_{A} \rightarrow T_{G}^{*} M_{X}$ above $i$. Then $i$ induces the morphisms $i^{*}: K_{G}^{0}\left(T_{G}^{*} M_{X}\right) \rightarrow K_{G}^{0}\left(\tilde{T}_{G}^{*} M_{A}\right)$ and $i_{!}: K_{G}^{0}\left(T_{G}^{*} M_{A}\right) \rightarrow K_{G}^{0}\left(T_{G}^{*} M_{X}\right)$. Combining these 3 maps, we claim that

Lemma 11.10 Take $\sigma \in K_{G}^{0}\left(T_{G}^{*} M_{X}\right)$, then $i_{!} C_{M_{B}^{\prime}}^{-1} i^{*}(\sigma)=\bigwedge_{-1} M_{B} \otimes \sigma$.

Since we are working on vector spaces, we can assume that all vector bundles are topologically trivial. Thus we can represent $\sigma$ as given by a variable linear map $\sigma(v, w, \xi, \eta): E \rightarrow F$ where $E, F$ are complex representation spaces, $v \in M_{A}, w \in$ $M_{B}, \xi \in M_{A}^{\prime}, \eta \in M_{B}^{\prime}$. Now $\sigma$ restricts to an element $\tilde{\sigma}:=i^{*} \sigma$ in $K_{G}^{0}\left(T_{G}^{*} M_{X} \mid M_{A}\right)$ which is represented by the map $\sigma(v, 0, \xi, \eta)$. Since $T_{G}^{*} M_{X} \mid M_{A}=T_{G}^{*} M_{A} \times M_{B}^{\prime}$, the element $\tilde{\sigma}$ is equivalent to $\mathbf{c}_{M_{B}^{\prime}} \odot q^{*} \tau$, where $q: T_{G}^{*} M_{A} \times M_{B}^{\prime} \rightarrow T_{G}^{*} M_{A}$ is the projection, $\tau$ a transversally elliptic symbol on $M_{A}$ and $\mathbf{c}_{M_{B}^{\prime}}$ the Bott symbol with support the zero section of the bundle $T_{G}^{*} M_{A} \times M_{B}^{\prime}$ on $T_{G}^{*} M_{A}$. Thus we have to show that $\bigwedge_{-1} M_{B} \otimes \sigma$ and $i_{!}(\tau)$ are homotopic.

By definition, a representative of the symbol $i_{!}(\tau)$ on $M_{A} \times M_{B}$ is the product of the symbol $\mathbf{c}_{M_{B} \otimes_{\mathbb{R}} \mathbb{C}}$ by the symbol $q^{*} \tau$. As $\mathbf{c}_{M_{B} \otimes_{\mathbb{R}} \mathbb{C}}=\mathbf{c}_{M_{B}} \odot \mathbf{c}_{M_{B}^{\prime}}$, we see that $i_{!}(\tau)=q^{*} \tau \odot \mathbf{c}_{M_{B}} \odot \mathbf{c}_{M_{B}^{\prime}}=\tilde{\sigma} \odot \mathbf{c}_{M_{B}}$.

As we have seen before, the symbol defined as $\bigwedge_{-1} M_{B} \otimes \sigma$ on the manifold $M_{A} \times$ $M_{B}$ is homotopic to the element $\mathbf{c}_{M_{B}} \odot \sigma$. Now consider the symbol $\sigma(t)(v, w, \xi, \eta)=$ $\sigma(v, t w, \xi, \eta)$ on $T^{*} M$. The intersection of the support of $\mathbf{c}_{M_{B}} \odot \sigma(t)$ with $T_{G}^{*} M$ stays compactly supported for all $t$. Indeed its support remains constant: this is the intersection of the support of $\sigma$ with $T_{G}^{*} M_{A}$. So we obtain the desired homotopy between $\bigwedge_{-1} M_{B} \otimes \sigma=\mathbf{c}_{M_{B}} \odot \sigma(1)$ and $i_{!}(\tau)=\mathbf{c}_{M_{B}} \odot \sigma(0)$ and the claim follows.

With the previous notations, $X=A \cup B, i: T_{G}^{*} M_{A} \times M_{B}^{\prime} \rightarrow T_{G}^{*} M_{X}$.

Corollary 11.11 Take $\sigma \in K_{G}^{0}\left(T_{G}^{*} M_{X}\right)$. Let $\sigma_{0}=C_{M_{B}^{\prime}}^{-1} i^{*}(\sigma) \in K_{G}^{0}\left(T_{G}^{*} M_{A}\right)$. Then, we have the equality of generalized functions on $G$ :

$$
\operatorname{det}_{M_{B}}(1-g) \operatorname{index}(\sigma)(g)=\operatorname{index}\left(\sigma_{0}\right)(g) .
$$

We are now ready to compare the exact sequence (35) with a combinatorial exact sequence using the index. Notice that $\tilde{Z}$ is a list of elements in $\Lambda / \mathbb{Z} a$ and functions on 
$\Lambda / \mathbb{Z} a$ cab be identified to functions on $\Lambda$ which are invariant under translation by $a$. We get

Theorem 28 The diagram

$$
\begin{aligned}
& 0 \rightarrow K_{G_{a}}^{0}\left(T_{G_{a}}^{*} M_{\tilde{Z}}^{f}\right) \stackrel{j_{*} k}{\longrightarrow} K_{G}^{0}\left(T_{G}^{*} M_{X}^{f}\right) \stackrel{C_{a}^{-1} i^{*}}{\longrightarrow} K_{G}^{0}\left(T_{G}^{*} M_{Z}^{f}\right) \rightarrow 0 \\
& \text { ind }_{m} \downarrow \quad \operatorname{ind}_{m} \downarrow \quad \quad \operatorname{ind}_{m} \downarrow \\
& 0 \longrightarrow D M(\tilde{Z}) \stackrel{i_{a}}{\longrightarrow} D M(X) \stackrel{\nabla_{a}}{\longrightarrow} \quad D M(Z) \rightarrow 0
\end{aligned}
$$

is commutative. Its vertical arrows are isomorphisms.

In particular, the index multiplicity map gives an isomorphism between $K_{G}^{0}\left(T_{G}^{*} M_{X}^{f}\right)$ and $\operatorname{DM}(X)$.

We start by remarking that, by Corollary 11.8 , all the vertical maps in our diagram are indeed taking values in the corresponding Dahmen-Micchelli spaces.

So we need to show commutativity. To prove the commutativity of the square on the right hand side, using Fourier transform, we need to prove that $\left(1-g^{a}\right) \operatorname{index}(\sigma)(g)=$ index $\left(C_{a}^{-1} i^{*}(\sigma)\right)(g)$.

From the symbol $\sigma$ on $M_{X}^{f}$, an open set in $M_{X}$, we deduce a symbol on $M_{X}$ with same index, by the excision property of the index. Thus the commutativity follows from Corollary 11.11 applied to $A=Z, B=\{a\}$. As for the square on the left hand side, since $j_{*}$ is an open embedding, it preserves indices. The statement thus follows from Proposition 11.4.

By induction we can then assume that the two external vertical arrows are isomorphism so, by the five Lemma, also the central one is and everything follows.

Summarizing we have isomorphisms

$$
K_{G}^{s+1}\left(M_{X}^{f}\right) \cong K_{G}^{1}\left(T_{G}^{*} M_{X}^{f}\right)=0, \quad K_{G}^{s}\left(M_{X}^{f}\right) \cong K_{G}^{0}\left(T_{G}^{*} M_{X}^{f}\right) \cong D M(X)
$$

Let us make two obvious remarks on the isomorphism $K_{G}^{0}\left(T_{G}^{*} M_{X}^{f}\right) \cong D M(X)$.

Remark 11.12 The space $K_{G}^{0}\left(T_{G}^{*} M_{X}^{f}\right)$ depends only of the manifold $M_{X}$ considered as a real manifold. The space $D M(X)$ depends only of the list $X$ up to change of signs.

Remark 11.13 If $U$ is a $G$ manifold, the index of an element $\sigma \in K_{G}^{0}\left(T_{G}^{*} U\right)$ is a generalized function supported on the set of points $g \in G$ such that $g$ has a fixed point in $U$.

We have seen in Sect. 8.3.1 that Fourier transforms of elements in $D M(X)$ are supported on the finite set of points $P(X)$. This is in agreement with the fixed point philosophy that we just recalled. In fact, an element $g \in G$ has a fixed point $v$ in $M_{X}^{f}$ if and only if $g \in P(X)$. Indeed if $g \in P(X)$, there exists a basis $\underline{b}$ of $V$ extracted form $X$ with $g^{b_{i}}=1$, for all $b_{i} \in \underline{b}$. Thus any element $v \in M_{X}$ with non zero coordinates on each $L_{b_{i}}$ is fixed by $g$, and is in $M_{X}^{f}$.

We now come to our next commutative diagram. 
Lemma 11.14 For each $s \geq i \geq 0$, the index multiplicity map ind sends $_{m}$ $K_{G}^{0}\left(T_{G}^{*} M_{\geq i}\right)$ to the space $\tilde{\mathcal{F}}_{i}(X)$.

Recall that $\tilde{\mathcal{F}}_{i}(X)$ is the subspace in $\tilde{\mathcal{F}}(X)$ such that $\nabla_{X \backslash \underline{t}} f=0$ for all $\underline{t} \in S_{X}^{(i-1)}$. Denote by $\ell: \tilde{\mathcal{F}}_{i}(X) \rightarrow \tilde{\mathcal{F}}(X)$ the inclusion.

By Corollary 11.9, if $\sigma \in K_{G}^{0}\left(T_{G}^{*} M_{\geq i}\right)$ and $\underline{t}$ is a rational subspace of dimension strictly less than $i$, we have $\bigwedge_{-1} M_{X \backslash t} \otimes \sigma=0$. Thus $\nabla_{X \backslash t} i n d_{m}(\sigma)=0$. It follows that the only thing we have to show is that, if $\sigma \in K_{G}^{0}\left(T_{G}^{*} M_{X}\right)$, then $\operatorname{ind}_{m}(\sigma)$ lies in $\tilde{\mathcal{F}}(X)$. Take a rational subspace $\underline{r}$. By Lemma 11.10, the index of $\bigwedge_{-1} M_{X \backslash \underline{r}} \otimes \sigma$ equals the index of an element $\sigma_{0} \in K_{G}^{0}\left(T_{G}^{*} M_{X \cap \underline{r}}\right)$. But the action of $G$ on $M_{X \cap r}$ factors though the quotient $G / G_{\underline{r}}$ whose character group is $\Lambda_{\underline{r}}$. Thus $K_{G}^{0}\left(T_{G}^{*} \bar{M}_{X \cap \underline{r}}\right) \cong R(G) \otimes_{R\left(G / G_{\underline{r}}\right)} K_{G / G_{\underline{\underline{r}}}}^{0}\left(T_{G / G_{\underline{\underline{r}}}}^{*} M_{X \cap \underline{r}}\right)$, hence $\nabla_{X \backslash \underline{r}} i n d_{m}(\sigma)=$ $\operatorname{ind}_{m}\left(\sigma_{0}\right)$ lies in $R(G) \otimes_{R\left(G / G_{r}\right)} \mathcal{C}\left[\Lambda_{\underline{r}}\right]$ as desired.

Our second commutative diagram and main theorem characterizes the values of the index on the entire $M_{X}$. This time, we use the notations and the exact sequences contained in Theorem 27 and Corollary 11.11.

Theorem 29 For each $0 \leq i \leq s$,

- the diagram

$$
\begin{aligned}
& 0 \rightarrow K_{G}^{0}\left(T_{G}^{*} M_{\geq i+1}\right) \stackrel{j_{*}}{\longrightarrow} K_{G}^{0}\left(T_{G}^{*} M_{\geq i}\right) \stackrel{C_{i}^{-1} e^{*}}{\longrightarrow} \quad K_{G}^{0}\left(T_{G}^{*} F_{i}\right) \rightarrow 0 \\
& \text { ind }_{m} \downarrow \quad \quad \quad \operatorname{ind}_{m} \downarrow \quad \text { ind } \downarrow \\
& 0 \rightarrow \tilde{\mathcal{F}}_{i+1}(X) \stackrel{\ell}{\longrightarrow} \tilde{\mathcal{F}}_{i}(X) \stackrel{\mu_{i}}{\longrightarrow} \oplus_{\underline{r} \in S_{X}^{(i)}} D M^{(G)}(X \cap \underline{r}) \rightarrow 0
\end{aligned}
$$

commutes.

- Its vertical arrows are isomorphisms.

- In particular, the index gives an isomorphism between $K_{G}^{0}\left(T_{G}^{*} M_{X}\right)$ and $\tilde{\mathcal{F}}(X)$.

Lemma 11.14 tells us that the diagram is well defined. We need to prove commutativity.

We prove that the square on the right hand side is commutative using Corollary 11.11. The square on the left hand side is commutative since $j_{*}$ is compatible with the index and $\ell$ is the inclusion.

Recall that $K_{G}^{0}\left(T_{G}^{*} M_{X \cap \underline{r}}\right) \cong R(G) \otimes_{R\left(G / G_{\underline{r}}\right)} K_{G / G_{\underline{r}}}^{0}\left(T_{G / G_{\underline{r}}}^{*} M_{X \cap \underline{r}}\right)$ and that $D M^{(G)}(X \cap \underline{r}) \cong R(G) \otimes_{R\left(G / G_{r}\right)} D M(X \cap \underline{r})$. Using Theorem 28, this implies that the right vertical arrow is always an isomorphism.

We then apply descending induction on $i$. When $i+1=s$, since $M_{\geq s}=M_{X}^{f}$ and $\tilde{\mathcal{F}}_{s-1}(X)=D M(X)$, Theorem 28 gives that the left vertical arrow is an isomorphism. So assume that the left vertical arrow is an isomorphism. We then deduce by the five Lemma that the central vertical arrow is an isomorphism and conclude by induction. 


\section{Generators}

In this section, we show that the generators of $\tilde{\mathcal{F}}(X)$ correspond via the index map to the generators of $K_{G}^{0}\left(T_{G}^{*} M_{X}\right)$ constructed by Atiyah-Singer in [1].

Recall that, given a manifold $M$, a way to construct elements of $K_{G}^{0}\left(T_{G}^{*} M\right)$ is to take a closed $G$-manifold $N$ embedded by $i: N \rightarrow M$. Then we have a map $i_{!}: K_{G}^{0}\left(T_{G}^{*} N\right) \rightarrow K_{G, c}^{0}\left(T_{G}^{*} M\right)$.

For our case $M=M_{X}$, we shall take the following manifolds. Take a flag $\phi$ of rational subspaces $0=\underline{r}_{0} \subset \underline{r}_{1} \subset \underline{r}_{2} \subset \cdots \subset \underline{r}_{s}$ with $\operatorname{dim}\left(\underline{r}_{i}\right)=i$ (and $s=\operatorname{dim} G$ ). Consider then the spaces $E_{i}:=\oplus_{a \in\left(X \cap \underline{r}_{i}\right) \backslash \underline{r}_{i-1}} L_{a}$. We choose an orientation for each $\underline{r}_{i}$ and divide the set of characters $Z_{i}:=\left(X \cap \underline{r}_{i}\right) \backslash \underline{r}_{i-1}$ into positive and negative elements $A_{i}, B_{i}$. Accordingly, we change the complex structure on each $L_{b}$ for which $b$ is negative into its conjugate structure. Let $A$ be the union of the sets $A_{i}$ and $B$ the union of the sets $B_{i}$. Choose a $G$-invariant Hermitian metric $h_{i}$ on $E_{i}$ and consider the unit sphere $S_{i}\left(h_{i}\right)$ on $E_{i}$. The product $S_{\phi}(h)=\prod_{i=1}^{s} S_{i}\left(h_{i}\right)$ is a closed submanifold of $M_{X}^{f}$.

The tangent space at a point $p$ of $S_{\phi}(h)$ decomposes as a vertical space generated by the rotations on each factor $E_{i}$ and the horizontal space $H_{p}$, a lift of the tangent space to the corresponding product of projective spaces. The horizontal space is a Hermitian vector space. The tangential Cauchy-Riemann operator $\delta_{\phi}$ is a differential operator on $S_{\phi}(h)$, the product of the operators $\delta_{i}$ described in Subsection 10.3. The index of $\delta_{\phi}$ is the product of the indices of $\delta_{i}$.

Let $c_{\phi}(p, \xi)=c\left(\xi^{1}\right)$ be the Clifford action on $\bigwedge H_{p}$ of the projection $\xi^{1}$ of $\xi$ on the horizontal tangent space $H_{p}$.

We then have [1] the following theorem.

\section{Theorem 30}

$$
\operatorname{index}\left(\delta_{\phi}\right)(g)=(-1)^{s}(-1)^{|B|} g^{\sum_{a \in A} a} \theta_{\phi}^{X}(g) .
$$

In fact $c_{\phi}=c_{1} \odot c_{2} \odot \cdots \odot c_{s}$ is the external product of the symbols $c_{i}$ of the operators $\delta_{i}$.

Let $i_{\phi}$ be the closed embedding of $S_{\phi}(h)$ in $M_{X}^{f}$. We can then give an "easy" proof of the following theorem of Atiyah-Singer (Theorem 7.9 of [1]).

Theorem 31 Let $i_{\phi}$ be the closed injection of $S_{\phi}(h)$ to $M_{X}$. Then the elements $\left(i_{\phi}\right) ! c_{\phi}$ generate $K_{G}^{0}\left(T_{G}^{*} M_{X}^{f}\right)$.

Consider $M_{X}$ as a real representation of $G$, each connected component $F$ of the space of regular elements in $\operatorname{Lie}(G)$ gives us a complex structure $J_{F}$ on $M_{X}$ and a corresponding "pushed" symbol $A t_{F}$ with $i n \operatorname{dex}\left(A t_{F}\right)(g)=(-1)^{|B|} g \sum_{a \in A} a_{\Theta}^{F}$ (Theorem 25).

Theorem 32 The symbols $A t_{F} \in K_{G}^{0}\left(T_{G}^{*} M_{X}\right)$, where $F$ varies over all open faces of the arrangement $\mathcal{H}_{X}$, give us a set of generators for $K_{G}^{0}\left(T_{G}^{*} M_{X}\right)$.

Remark 12.1 After checking naturality axioms, Theorem 32 reduces the proof of the cohomological index formula given by [9] or [30] to the case of the symbols $A t_{F}$. 
So Theorem 32 is crucial in establishing a cohomological formula valid for any transversally elliptic operator.

\section{Equivariant cohomology and infdex}

The purpose of this section is to establish cohomological formulas for the index of transversally elliptic operators (cf. [22] and [21]). We do this through a new invariant, the infinitesimal index.

This construction is motivated by taking the Fourier transform of the formula of Berline-Vergne for the equivariant index of a transversally elliptic operator [8,9,27] where one can also find the various notations and definitions. As we shall see the combinatorial spaces $D M(X)$ and $\mathcal{F}(X)$ appearing in $K$-theory and index Theory will be replaced by spaces of distributions completely analogues to the ones appearing in the Theory of splines and giving rise to precise analogues.

\subsection{Equivariant de Rham cohomology}

Let $M$ be a $C^{\infty}$ manifold with a $C^{\infty}$ action of a compact Lie group $G$, we are going to define its equivariant cohomology with compact support following Cartan (see [24]).

We define the space of compactly supported equivariant forms as

$$
\mathcal{A}_{G, c}(M)=\left(S\left(\mathfrak{g}^{*}\right) \otimes \mathcal{A}_{c}(M)\right)^{G}
$$

with the grading given setting $\mathfrak{g}^{*}$ in degree 2 . Here $\mathcal{A}_{c}(M)$ is the algebra of differential forms on $M$ with compact support.

Each element $x \in \mathfrak{g}$ of the Lie algebra of $G$ induces a vector field $v_{x}$ on $M$, the infinitesimal generator of the action: here the sign convention is that $v_{x}=\frac{d}{d \epsilon} \exp (-\epsilon x) \cdot m$ in order that the map $x \rightarrow v_{x}$ be a Lie algebra homomorphism. A vector field $V$ on $M$ induces a derivation $\iota_{V}$ on forms, such that $\iota_{V}(d f)=V(f)$ and for simplicity we denote by $\iota_{x}=\iota_{v_{x}}$.

One defines the differential as follows. Given $\alpha \in \mathcal{A}_{G, c}(M)$, we think of $\alpha$ as an equivariant polynomial map on $\mathfrak{g}$ with values in $\mathcal{A}_{c}(M)$, thus for any $x \in \mathfrak{g}$ we set

$$
D \alpha(x):=d(\alpha(x))-\iota_{x}(\alpha(x))
$$

where $d$ is the usual de Rham differential.

It is easy to see that $D$ increases the degree by one and that $D^{2}=0$. Thus we can take cohomology and we get the $G$-equivariant cohomology of $M$ with compact support.

Now take a $G$-stable closed set $Z$ in a manifold $M$. Consider the open set $U=M \backslash Z$. Then $U$ is a manifold and we have an inclusion of complexes $\mathcal{A}_{G, c}(U) \subset \mathcal{A}_{G, c}(M)$ given by extension by zero. We set

$$
\mathcal{A}_{G, c}(Z, M):=\mathcal{A}_{G, c}(M) / \mathcal{A}_{G, c}(U)
$$


Definition 13.2 The equivariant de Rham cohomology with compact support $H_{G, c}^{*}(Z)$ is the cohomology of the complex $\mathcal{A}_{G, c}(Z, M)$.

Notice that $\mathcal{A}_{G, c}(U)$ is an ideal in $\mathcal{A}_{G, c}(M)$ so $\mathcal{A}_{G, c}(Z, M)$ is a differential graded algebra and $H_{G, c}^{*}(Z)$ is a graded algebra (without 1 if $Z$ is not compact).

In this model, a representative of a class in $H_{G, c}^{*}(Z)$ is an equivariant form $\alpha(x)$ with compact support on $M$. The form $\alpha$ is not necessary equivariantly closed on $M$, but there exists a neighborhood of $Z$ such that the restriction of $\alpha(x)$ to this neighborhood is equivariantly closed.

\subsection{Action form and the moment map}

Let $G$ be a Lie group and $M$ a $G$-manifold.

Definition 13.4 An action form is a $G$-invariant real one form $\sigma$ on $M$.

The prime examples of this setting are when $M$ is even dimensional and $d \sigma$ is non degenerate. In this case $d \sigma$ defines a symplectic structure on $M$.

Example 13.5 For every manifold $N$, we may take its cotangent bundle $M:=T^{*} N$ with projection $\pi: T^{*} N \rightarrow N$. The canonical action form $\sigma$ on a tangent vector $v$ at a point $(n, \phi), n \in N, \phi \in T_{n}^{*} N$ is given by

$$
\langle\sigma \mid v\rangle:=\langle\phi \mid d \pi(v)\rangle
$$

In this setting, $d \sigma$ is a canonical symplectic structure on $T^{*} N$ and, if $r=\operatorname{dim}(N)$, the form $\frac{d \sigma^{r}}{r !}$ determines an orientation and a measure, the Liouville measure on $T^{*} N$. If a group $G$ acts on $N$, then it acts also on $T^{*} N$ preserving the canonical action form and hence the symplectic structure and the Liouville measure.

Remark 13.6 If $M$ is a manifold with a $G$-invariant Riemannian structure, we can consider an invariant vector field instead of a 1-form.

Let $v_{x}$ be the vector field on $M$ associated to $x \in \mathfrak{g}$ and $i_{x}$ the derivation on forms induced by contraction with $v_{x}$.

Definition 13.7 Given an action form $\sigma$ we define the moment map $\mu_{\sigma}: M \rightarrow \mathfrak{g}^{*}$ associated to $\sigma$ by:

$$
\mu_{\sigma}(m)(x):=-\left\langle\sigma \mid v_{x}\right\rangle(m)=-\iota_{x}(\sigma)(m) .
$$

for $m \in M, x \in \mathfrak{g}$.

The moment map is related to the equivariant differential of $\sigma$ as defined in the Cartan model (see Formula (38)).

$$
\Omega(x)=D \sigma(x)=\mu(x)+d \sigma .
$$


13.8 Infinitesimal index

We fix a translation invariant Lebesgue measure $d \xi$ on $\mathfrak{g}^{*}$. We choose a square root $i$ of -1 and define the Fourier transform:

$$
\hat{f}(x):=\int_{\mathfrak{g}^{*}} e^{-i\langle\xi \mid x\rangle} f(\xi) d \xi
$$

We normalize $d x$ on $\mathfrak{g}$ so that the inverse Fourier transform is

$$
f(\xi)=\int_{\mathfrak{g}} e^{i\langle\xi \mid x\rangle} \hat{f}(x) d x
$$

The measure $d x d \xi$ is independent of the choice of $d \xi$.

If $f(\xi)$ is a $C^{\infty}$ function on $\mathfrak{g}^{*}$ with compact support in a ball $B_{R}$ of radius $R$ in $\mathfrak{g}^{*}$ (for a choice of Euclidean structure on $\mathfrak{g}^{*}$ ) its Fourier transform $\hat{f}(x)$ is a rapidly decreasing function on $\mathfrak{g}$.

Theorem 33 Let $\sigma$ be an action form with moment map $\mu$. Let $M^{0}=\mu^{-1}(0)$. Then we can define a map

$$
\operatorname{infdex}_{G}^{\sigma}: H_{G, c}^{*}\left(M^{0}\right) \rightarrow \mathcal{D}^{\prime}\left(\mathfrak{g}^{*}\right)^{G}
$$

setting for any $[\alpha] \in H_{G, c}^{*}\left(M^{0}\right)$ and for any smooth function with compact support $f$ on $\mathfrak{g}^{*}$

$$
\left\langle\operatorname{infdex}_{G}^{\sigma}([\alpha]), f\right\rangle:=\lim _{s \rightarrow \infty} \int_{M} \int_{\mathfrak{g}} e^{i s \Omega(x)} \alpha(x) \hat{f}(x) d x .
$$

The map $\operatorname{infdex}_{G}^{\sigma}$ is a well defined homomorphism of $S\left[\mathfrak{g}^{*}\right]^{G}$ modules.

If the one form $\sigma$ moves along a smooth curve $\sigma_{t}$ with moment map $\mu_{t}$ such that $\mu_{t}^{-1}(0)$ remains equal to $M^{0}$, then

$$
\operatorname{infdex}_{G}^{\sigma_{t}}=\operatorname{infdex}_{G}^{\sigma}
$$

13.8.1. Applications. We apply the Theory to a linear representation $M_{X}$ of a torus, with list of characters $X$. Then we have, for the infinitesimal index, cohomological analogues of the $K$-theoretical results 28 and 29. on the index discussed in Sect. 11.

Theorem 34 The map infdex is a graded isomorphism as $S\left[\mathfrak{g}^{*}\right]$-modules of $H_{G, c}^{*}\left(T_{G}^{*} M_{X}^{\text {fin }}\right)$ onto $D(X)$.

Finally as for $K$-theory and the index we have 
Theorem 35 For each $0 \leq i \leq s-1$,

(a) For each $h \geq 0, H_{G, c}^{2 h+1}\left(T_{G}^{*} M_{X, \geq i}\right)=0$.

(b) For each $h \geq 1$, the following sequence is exact

$$
0 \rightarrow H_{G, c}^{2 h}\left(T_{G}^{*} M_{X, \geq i+1}\right) \stackrel{j_{*}}{\rightarrow} H_{G, c}^{2 h}\left(T_{G}^{*} M_{X, \geq i}\right) \stackrel{C_{i}^{-1} e^{*}}{\rightarrow} \bigoplus_{\underline{s} \in \mathcal{S}_{X}(i)} H_{G, c}^{2 h-2|X \backslash \underline{s}|}\left(T_{G}^{*} M_{\underline{s}}^{f}\right) \rightarrow 0 .
$$

Let $\underline{r}$ be a vector subspace in $\mathfrak{g}^{*}$. We have an embedding $j: \mathcal{S}^{\prime}(\underline{r}) \rightarrow \mathcal{S}^{\prime}\left(\mathfrak{g}^{*}\right)$ by $j(\phi)(f)=\phi(f \mid \underline{r})$ for any $\phi \in \mathcal{S}^{\prime}(\underline{r}), f$ a Schwartz function on $\mathfrak{g}^{*}$. We denote the image $j\left(\mathcal{S}^{\prime}(\underline{r})\right)$ by $\mathcal{S}^{\prime}\left(\mathfrak{g}^{*}, \underline{r}\right)$ (sometimes we even identify $\mathcal{S}^{\prime}(\underline{r})$ with $\mathcal{S}^{\prime}\left(\mathfrak{g}^{*}, \underline{r}\right)$ if there is no ambiguity). We next define the vector space:

\section{Definition 13.9}

$$
\mathcal{G}(X):=\left\{f \in \mathcal{S}^{\prime}\left(\mathfrak{g}^{*}\right) \mid \partial_{X \backslash \underline{r}} f \in \mathcal{S}^{\prime}\left(\mathfrak{g}^{*}, \underline{r}\right) \text {, for all } \underline{r} \in \mathcal{S}_{X}\right\}
$$

The following theorem characterizes the values of the infinitesimal index on the entire $M_{X}$. This time, we use the notations and the exact sequences contained in Theorem 27 and Corollary 11.11.

Theorem 36 For each $0 \leq i \leq s$,

- the diagram

$$
\begin{aligned}
& 0 \rightarrow H_{G, c}^{*}\left(T_{G}^{*} M_{X, \geq i+1}\right) \stackrel{j_{*}}{\longrightarrow} H_{G, c}^{*}\left(T_{G}^{*} M_{X, \geq i}\right) \stackrel{C_{i}^{-1} e^{*}}{\longrightarrow} \quad H_{G, c}^{*}\left(T_{G}^{*} M_{=i}\right) \rightarrow 0 \\
& \text { infdex } \downarrow \quad \text { infdex } \downarrow \text { infdex } \downarrow \\
& 0 \rightarrow \tilde{\mathcal{G}}_{i+1}(X) \stackrel{\ell}{\longrightarrow} \quad \tilde{\mathcal{G}}_{i}(X) \stackrel{\mu_{i}}{\longrightarrow} \oplus_{\underline{s} \in \mathcal{S}_{X}(i)} D^{\mathfrak{g}}(X \cap \underline{s}) \rightarrow 0
\end{aligned}
$$

commutes.

- Its vertical arrows are isomorphisms.

- In particular, the infinitesimal index gives an isomorphism between $H_{G, c}^{*}\left(T_{G}^{*} M_{X}\right)$ and $\tilde{\mathcal{G}}(X)$.

13.9.1. Index and infinitesimal index. Our final task is to relate all commutative diagrams, of index and $K$-theory on one hand, infinitesimal index and cohomology on the other, in a unique commutative diagram involving the Chern character on one hand and Poisson summation formula on the other. The box spline now appears in Fourier transform since the following result is well known.

$$
\operatorname{ch}\left(\operatorname{Bott}\left(M_{X}\right)\right)(x)=(2 i \pi)^{|X|} \prod_{a \in X} \frac{e^{i\langle a, x\rangle}-1}{i\langle a, x\rangle} \operatorname{Thom}\left(M_{X}\right)(x)
$$

in the cohomology group of smooth equivariant differential forms.

We denote by $X_{R}$ the sequence $X \cup-X$ of characters. Remark that the zonotope associated to $X_{R}$ contains 0 in its closure. 
Theorem 37 (Convolution theorem) Let $X \subset \Lambda$ be a system of characters of $G$. Let

$$
X_{R}=X \cup-X
$$

Let $\Sigma$ be a $G$-invariant transversally elliptic symbol on $M$. Let $\operatorname{ind}_{m}(\Sigma) \in \mathcal{C}_{\mathbb{Z}}[\Lambda]$ be its multiplicity index. Let $\operatorname{infdex}_{G}^{-\mu}(\operatorname{ch}(\Sigma))$ be the infinitesimal index of its Chern character. Then

$$
B_{X_{R}} *_{d} \operatorname{ind}_{m}(\Sigma)=(2 i \pi)^{-2|X|} \operatorname{infdex}_{G}^{-\mu} \operatorname{ch}(\Sigma)
$$

We need thus a Deconvolution theorem which we state in the unimodular case (for the general case see [22] and [21]).

Theorem 38 (Deconvolution theorem) Assume that $X$ is unimodular. Let $\mathfrak{c}$ be an alcove in $V$ containing 0 in its closure and contained in $Z(X)$. Then

(i) $\lim _{\mathfrak{c}}\left(\operatorname{Todd}(X)_{p w} B_{X}\right)=\delta_{0}$.

(ii) For any $K \in \mathcal{C}[\Lambda]$,

$$
K=\lim _{\mathfrak{c}}\left(\operatorname{Todd}(X)_{p w}\left(B_{X} *_{d} K\right)\right) .
$$

Using the deconvolution theorem in the unimodular case, Theorem 37 leads to the following theorem, which is strongly reminiscent of the Riemann-Roch theorem. Remark that as $X_{R}$ contains 0 in its interior, we may use any alcove containing 0 in its closure in the limiting procedure.

We denote by $\operatorname{Todd}\left(X_{R}\right)$ the Todd operator associated to $X_{R}$. It acts on the space of piecewise polynomial functions for the system $(X, \Lambda)$.

Theorem 39 Let $X \subset \Lambda$ be a unimodular system of characters of $G$. Let

$$
\operatorname{Todd}\left(X_{R}\right)=\prod_{a \in X \cup-X} \frac{\partial_{a}}{1-e^{-\partial_{a}}}
$$

be the Todd operator.

Let $\Sigma$ be a $G$-invariant transversally elliptic symbol on $M, \operatorname{ind}_{m}(\Sigma) \in \mathcal{C}_{\mathbb{Z}}[\Lambda]$ be its multiplicity index and $\operatorname{infdex}_{G}^{-\mu}(\operatorname{ch}(\Sigma))$ be the infinitesimal index of its Chern character. Then

- $\operatorname{infdex}_{G}^{-\mu}(\operatorname{ch} \Sigma)$ is a piecewise polynomial measure on $\mathfrak{g}^{*}$.

- Let $\mathfrak{c}$ be an alcove having 0 in its closure. We have

$$
\operatorname{ind}_{m}(\Sigma)=(2 i \pi)^{-2|X|} \lim _{\mathfrak{c}} \operatorname{Todd}\left(X_{R}\right)_{p w} \operatorname{infdex}_{G}^{-\mu}(\operatorname{ch}(\Sigma)) \text {. }
$$

Remark 13.10 It is possible to show in this unimodular case that the piecewise polynomial function $(2 i \pi)^{-2|X|} \operatorname{Todd}\left(X_{R}\right)_{p w} \operatorname{infdex}_{G}^{-\mu}(\operatorname{ch}(\Sigma))$ extends to a continuous function on $\mathfrak{g}^{*}$. Thus its restriction to $\Lambda$ gives the index multiplicity. 
We formulate now the general index theorem. We denote by $P(X) \subset G$ the points of the arrangement.

Theorem 40 Let $X$ be a sequence of elements in $\Lambda$ and let $M:=M_{X}$. Let

$$
X_{R}=X \cup-X
$$

Let $\Sigma$ be a $G$-invariant transversally elliptic symbol on $M$ and $\operatorname{ind}_{m}(\Sigma) \in \mathcal{C}_{\mathbb{Z}}[\Lambda]$ be its multiplicity index.

For any $g \in P(X)$, let $\operatorname{infdex}_{G}^{-\mu}\left(\operatorname{ch}^{g}(\Sigma)\right)$ be the distribution on $\mathfrak{g}^{*}$ associated to the cohomology class $\operatorname{ch}^{g}(\Sigma) \in \mathcal{H}_{G, c}^{\infty, m}\left(T_{G}^{*} M^{g}\right)$ by the infinitesimal index. Then

- $\operatorname{infdex}_{G}^{-\mu}\left(\operatorname{ch}^{g}(\Sigma)\right)$ is a piecewise polynomial measure on $\mathfrak{g}^{*}$.

- Let $\mathrm{c}$ be an alcove having 0 in its closure. We have

$$
\operatorname{ind}_{m}(\Sigma)=\sum_{g \in P(X)}(2 i \pi)^{-2\left|X^{g}\right|} \hat{g} \lim _{\mathfrak{c}} D\left(X_{R} \backslash X_{R}^{g}, g\right)^{-1}
$$

$$
\operatorname{Todd}\left(X_{R}^{g}\right) * p w \operatorname{infdex}_{G}^{-\mu}\left(\operatorname{ch}^{g^{-1}}(\Sigma)\right) .
$$

Open Access This article is distributed under the terms of the Creative Commons Attribution License which permits any use, distribution and reproduction in any medium, provided the original author(s) and source are credited.

\section{References}

1. Atiyah, M.: Elliptic operators and compact groups. In: Lecture Notes in Mathematics, vol. 201. Springer, Berlin (1974)

2. Atiyah, M.F.: $K$-theory. Advanced Book Classics, 2nd edn. Addison-Wesley Publishing Company Advanced Book Program, Redwood City (1989). Notes by D.W. Anderson

3. Atiyah, M.F., Singer, I.M.: The index of elliptic operators. I. Ann. Math. 87, 484-530 (1968)

4. Atiyah, M.F., Singer, I.M.: The index of elliptic operators. III. Ann. Math. (2) 87, 546-604 (1968)

5. Atiyah, M.F., Singer, I.M.: Index theory for skew-adjoint Fredholm operators. Inst. Hautes Études Sci. Publ. Math. 37, 5-26 (1969)

6. Atiyah, M.F., Singer, I.M.: The index of elliptic operators. IV . Ann. Math. (2) 93, 119-138 (1971)

7. Atiyah, M.F., Singer, I.M.: The index of elliptic operators. V. Ann. Math. (2) 93, 139-149 (1971)

8. Berline, N., Vergne, M.: The Chern character of a transversally elliptic symbol and the equivariant index. Invent. Math. 124(1-3), 11-49 (1996)

9. Berline, N., Vergne, M.: L'indice équivariant des opérateurs transversalement elliptiques. Invent. Math. 124(1-3), 51-101 (1996)

10. Brion, M., Vergne, M.: Residue formulae, vector partition functions and lattice points in rational polytopes. J. Am. Math. Soc. 10(4), 797-833 (1997)

11. Brion, M., Vergne, M.: Arrangement of hyperplanes. I. Rational functions and Jeffrey-Kirwan residue. Ann. Sci. École Norm. Sup. (4) 32(5), 715-741 (1999)

12. Coutinho, S.C.: A primer of algebraic $D$-modules. In: London Mathematical Society Student Texts, vol. 33. Cambridge University Press, Cambridge (1995)

13. Dahmen, W., Micchelli, C.A.: Translates of multivariate splines. Linear Algebra Appl. 52/53, 217234 (1983) 
14. Dahmen, W., Micchelli, C.A.: The number of solutions to linear Diophantine equations and multivariate splines. Trans. Am. Math. Soc. 308(2), 509-532 (1988)

15. de Boor, C., Höllig, K., Riemenschneider, S.: Box splines. In: Applied Mathematical Sciences, vol. 98. Springer, New York (1993)

16. De Concini, C., Procesi, C.: Wonderful models of subspace arrangements. Selecta Math. (N.S.) 1(3), 459-494 (1995)

17. De Concini, C., Procesi, C.: Nested sets and Jeffrey-Kirwan residues. In: Geometric Methods in Algebra and Number Theory. Progr. Math., vol. 235, pp. 139-149. Birkhäuser Boston, Boston (2005)

18. De Concini, C., Procesi, C., Vergne, M.: The infinitesimal index. arXiv:1003.3525

19. De Concini, C., Procesi, C., Vergne, M.: Partition function and generalized Dahmen-Micchelli spaces. Transf. Groups 15(4), 751-773 (2010)

20. De Concini, C., Procesi, C., Vergne, M.: Vector partition functions and index of transversally elliptic operators. Transf. Groups 15(4), 775-811 (2010)

21. De Concini, C., Procesi, C., Vergne, M.: Box splines and the equivariant index theorem. arXiv:1012. 1049

22. De Concini, C., Procesi, C., Vergne, M.: Infinitesimal index: cohomology computations. arXiv:1005. 0128

23. De Concini, C., Procesi, C.: Topics in Hyperplane Arrangements, Polytopes and Box-Splines. Universitext. Springer, New York (2011)

24. Guillemin, V.W., Sternberg, S.: Supersymmetry and equivariant de Rham theory. In: Mathematics Past and Present. Springer, Berlin (1999). With an appendix containing two reprints by Henri Cartan [MR0042426 (13,107e); MR0042427 (13,107f)]

25. Hirzebruch, F.: Topological methods in algebraic geometry. In: Classics in Mathematics. Springer, Berlin (1995). Translated from the German and Appendix One by R.L.E. Schwarzenberger, With a preface to the third English edition by the author and Schwarzenberger, Appendix Two by A. Borel, Reprint of the 1978 edition

26. Jeffrey, L.C., Kirwan, F.C.: Localization for nonabelian group actions. Topology 34(2), 291-327 (1995)

27. Paradan, P.-E., Vergne, M.: The index of transversally elliptic operators. Bull. Soc. Math. France. (to appear). arXiv:0804.1225

28. Paradan, P.-É.: Jump formulas in Hamiltonian geometry. arXiv:math/0411306

29. Paradan, P.-É.: Localization of the Riemann-Roch character. J. Funct. Anal. 187, 442-509 (2001)

30. Paradan, P.-É., Vergne, M.: Index of transversally elliptic operators. arXiv:math 0805.1225

31. Schoenberg, I.J.: Cardinal Spline Interpolation. Society for Industrial and Applied Mathematics, Philadelphia (1973). Conference Board of the Mathematical Sciences Regional Conference Series in Applied Mathematics, No. 12

32. Segal, G.: Equivariant $K$-theory. Inst. Hautes Études Sci. Publ. Math. 34, 129-151 (1968)

33. Strang, G., Fix, G.J.: An Analysis of the Finite Element Method. Prentice-Hall Inc., Englewood Cliffs (1973). Prentice-Hall Series in Automatic Computation

34. Szenes, A., Vergne, M.: Residue formulae for vector partitions and Euler-MacLaurin sums. Adv. Appl. Math. 30(1-2), 295-342 (2003). Formal Power Series and Algebraic Combinatorics (Scottsdale, AZ, 2001) 